\title{
Exploratory Use of Microaerosol Decontamination Technology (PAEROSOL) in Enclosed, Unoccupied Hospital Setting
}

\author{
El Rainina \\ M Luna \\ P Godoy-Kain
}

May 2012

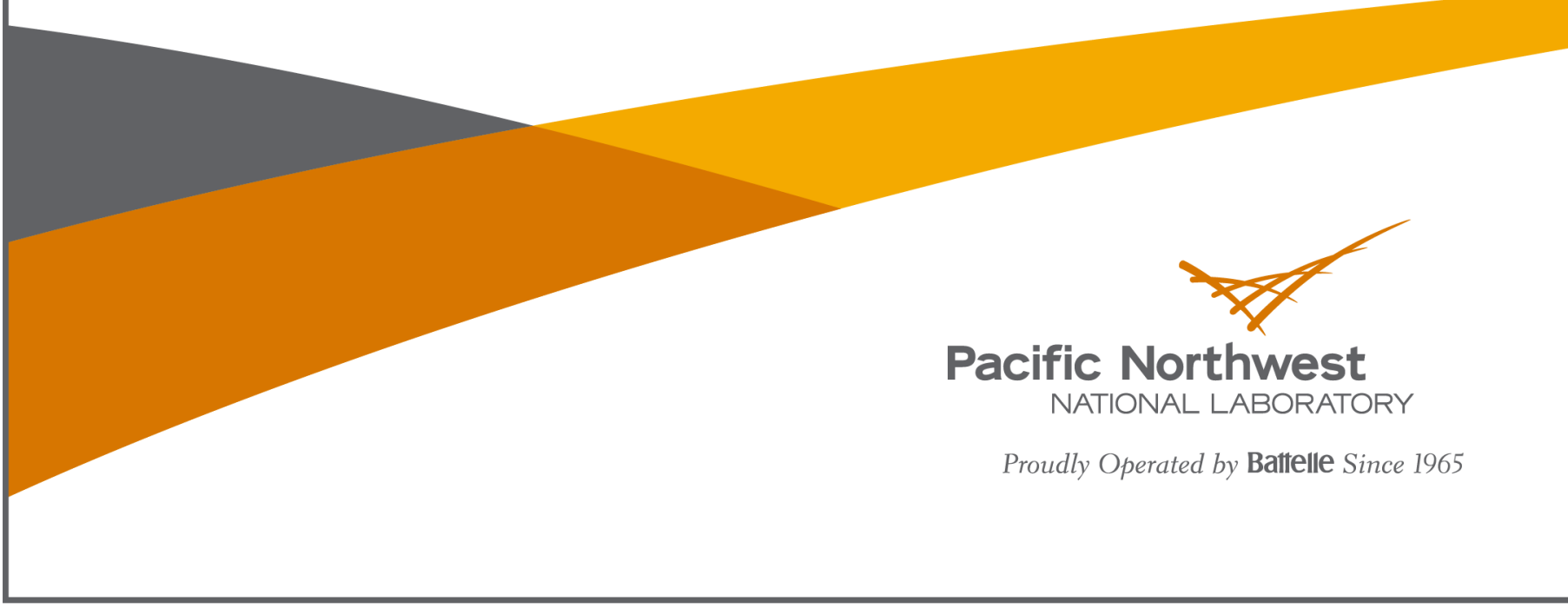




\title{
DISCLAIMER
}

This report was prepared as an account of work sponsored by an agency of the United States Government. Neither the United States Government nor any agency thereof, nor Battelle Memorial Institute, nor any of their employees, makes any warranty, express or implied, or assumes any legal liability or responsibility for the accuracy, completeness, or usefulness of any information, apparatus, product, or process disclosed, or represents that its use would not infringe privately owned rights. Reference herein to any specific commercial product, process, or service by trade name, trademark, manufacturer, or otherwise does not necessarily constitute or imply its endorsement, recommendation, or favoring by the United States Government or any agency thereof, or Battelle Memorial Institute. The views and opinions of authors expressed herein do not necessarily state or reflect those of the United States Government or any agency thereof.

\author{
PACIFIC NORTHWEST NATIONAL LABORATORY \\ operated by \\ BATTELLE \\ for the \\ UNITED STATES DEPARTMENT OF ENERGY \\ under Contract DE-AC05-76RL01830 \\ Printed in the United States of America \\ Available to DOE and DOE contractors from the \\ Office of Scientific and Technical Information, \\ P.O. Box 62, Oak Ridge, TN 37831-0062; \\ ph: (865) 576-8401 \\ fax: $(865)$ 576-5728 \\ email: reports(a)adonis.osti.gov \\ Available to the public from the National Technical Information Service \\ 5301 Shawnee Rd., Alexandria, VA 22312 \\ ph: (800) 553-NTIS (6847) \\ email: orders@intis.gov $<$ http://www.ntis.gov/about/form.aspx $>$ \\ Online ordering: http://www.ntis.gov
}

This document was printed on recycled paper.

$(8 / 2010)$ 


\title{
Exploratory Use of Microaerosol Decontamination Technology (PAEROSOL) in Enclosed, Unoccupied Hospital Setting
}

\author{
EI Rainina \\ M Luna \\ P Godoy-Kain
}

May 2012

Prepared for

The Defense Threat Reduction Agency under a

Government Order with the U.S. Department of Energy

Contract DE-AC05-76RL01830 



\section{Executive Summary}

A recent Centers for Disease Control and Prevention report estimated that annually 1.7 million patients acquire an infection while in a hospital (known as healthcare-associated infections, or HAIs), and the annual medical costs of healthcare associated with HAIs in U.S. hospitals are estimated to be between $\$ 5$ billion and $\$ 11$ billion. In other words, the patients contracted the infection while in the hospital, and the infection was not a result of their medical condition when admitted to the hospital. It is estimated that about 90,000 individuals die every year from HAIs. The list of HAIs is extensive; some are relatively easy to treat and some, like methicillin-resistant Staphylococcus aureus (MRSA), are life-threatening to both patients and staff. At least one third of HAIs are considered preventable and can be directly related to a standard of practice in infection control cleaning techniques. Although important in reducing surface contamination, manual cleaning methods have limited efficacy in reducing the bioburden on the surfaces beyond "high touch" areas, and they are completely ineffective in disinfecting airborne pathogens disseminated via aerosols in healthcare environments. Therefore new, more effective methods are needed to enhance the decontamination of various hospital facilities where diseases can rapidly and easily spread.

Alternative disinfection technologies such as gaseous decontaminations are being marketed to the healthcare sector. Gaseous decontamination offers a complementary technology to manual cleaning that increases the probability of an effective reduction in pathogens by providing a comparatively uniform distribution of disinfectant in an entire confined environment. Three commercially available gaseous decontamination technologies have been extensively examined and reported as effective in reducing HAIs: gaseous hydrogen peroxide, chlorine dioxide, and ozone. While there is a role to play for these new technologies in the decontamination of healthcare settings, the requirement for both a thorough preclean of surfaces and safety measures to ensure no gases and vapors penetrate beyond the space being decontaminated calls into question the safety and cost-effectiveness of these methods in healthcare facilities.

The goal of this study was to validate the previously observed high biological kill performance of the PAEROSOL decontamination technology against common HAIs in a non-human subject trial within a hospital setting of Madigan Army Medical Center (MAMC) on Joint Base Lewis-McChord in Tacoma, Washington. PAEROSOL - a semi-dry micro-aerosol atomized from a $0.5-0.25 \%$ aqueous solution of table salt that has been electrochemically activated - was previously shown to be non-toxic, environmentally safe, and requiring no precautions for its application. As compared to gaseous hydrogen peroxide, chlorine dioxide, and ozone, PAEROSOL application requires neither thorough pre-cleaning of the surfaces, nor special safety measures. In addition to validating the disinfecting efficacy of PAEROSOL against HAI pathogens on interior materials characteristic of hospital inanimate surfaces, the objectives of the trial included a demonstration of PAEROSOL environmental safety, (i.e., impact to hospital interior materials and electronic equipment exposed during testing) and PAEROSOL parameters optimization for future deployment.

The trial was performed inside the MAMC Department of Clinical Investigation Animal Surgical Suite. The volume of the test room (post-mortem room) was approximately $2000 \mathrm{ft}^{3}$ and it contained the normal fixtures of an animal post-mortem room: a surgical table, washing facilities, shelves, cabinets, a table, chairs, surgical ceiling lamp, and posters. The room also contained electronic equipment, such as a pH meter, a STEL electrochemical device, humidity/temperature sensors, and a peristaltic pump. 
Based on a published MAMC antibiogram (2009), Klebsiella pneumoniae, Pseudomonas aeruginosa, and Staphylococcus aureus, were selected for this trial and provided by MAMC. In addition, MAMC recommended using Bacillus subtilis spores to mimic Clostridium difficile spores. MAMC also selected and supplied the samples of flooring tile and carpet, countertop material, and privacy curtain fabric that are in use at MAMC. Upon MAMC recommendation, PAEROSOL bactericidal efficacy was tested against pathogen load on the samples of hospital materials in the range of $10^{7}$ to $10^{9}$ colony-forming units (CFU) per square meter.

The study used approximately $350,1-$ in. $^{2}$ coupons of each material. Each coupon was inoculated with $100 \mu 1$ of bacterial suspension in tryptic soy broth (which acted as an organic soil load) to achieve $1 \times 10^{3}$ to $1 \times 10^{5} \mathrm{CFU}$ per coupon. The spores were suspended in distilled water to prevent spore germination during coupon inoculation. The inoculated coupons were allowed to dry at room temperature while resting in uncovered sterile Petri dishes (each coupon in an individual Petri dish) for approximately 1-2 hours in a biological safety cabinet (BSC). Once the coupons were dry, the Petri dishes were covered with lids and stored in BSC before use. Before the experiment, inoculated coupons in Petri dishes were moved to the test room in special containers. In the test room, uncovered Petri dishes were positioned according to the use of the coupon material: the tile and carpet coupons were placed on the floor; the countertop coupons were placed on the tables, and the curtain cloth coupons were pinned to ribbons and positioned vertically, as curtains would hang in a hospital cubicle. In addition to the coupons, each tested culture $\left(1 \times 10^{4}\right.$ to $\left.1 \times 10^{5} \mathrm{CFU}\right)$ was inoculated on tryptic soy agar (TSA) or on TSA containing 5 percent sheep blood (TSA-5\% SB), which acted as heavy organic soil load. To assess PAEROSOL decontaminating efficacy under realistic conditions, each microbial culture inoculated on each material was positioned in three locations inside the room, including locations where PAEROSOL dispersion might be obstructed (e.g., under the table, and under the shelves). Petri dishes with the cultures inoculated directly on TSA and TSA-5\% SB were positioned near the coupons.

The PAEROSOL disinfecting microaerosol (particle size in the range of $0.5 \mu-10 \mu$ ) was generated inside the test room using a portable vortical aerosol generator (VAG) connected to an in-room compressed air line (35 psi) through a simple ASCO solenoid, which allowed the remote control of the VAG through the closed door. The liquid disinfectant for PAEROSOL production was prepared onsite from an aqueous solution of table salt $(\mathrm{NaCl})$ using a portable STEL-electrochemical device.

During the experiment, the furniture and electronic equipment remained inside the test room as they would under normal-use circumstances. The door to the rest room was closed but not sealed, and the airhandling system was turned off.

Analysis of inoculated coupons and Petri dishes pre-positioned in the test room determined the decontaminating efficacy of the PAEROSOL dispersed inside the test room.

Immediately after the PAEROSOL treatment of the room, the microbes were extracted from the coupons, appropriately diluted, plated on TSA or TSA-5\% SB, grown for 18-30 hours (depending on microbial culture), and grown colonies were counted to assess the CFU that remained on the coupons. Exposed Petri dishes were placed in an incubator, grown during the time appropriate for each culture, and then colonies were counted. Multiple control scenarios were applied. Each sample was used once and disposed after the experiment was complete, in accordance with best laboratory practice. 
To optimize PAEROSOL parameters for future deployment, three independent variables were tested and observed in the trial: 1) sodium chloride concentration for PAEROSOL production; 2) the volume of PAEROSOL dispersed inside the room; and 3) the time necessary to complete disinfection. Room temperature, humidity, and the concentration of oxidative species inside the test room were monitored but not controlled during each experiment. To evaluate the interactions between these variables and PAEROSOL efficacy, we ran serial experiments altering these variables. Outcome analyses assessed the effectiveness of the PAEROSOL application on reducing the number of viable microorganisms on test samples compared to the natural loss of viability of microbes on test coupons that were not exposed to PAEROSOL.

The most optimal conditions for reducing bacterial viability were applied to prepare a final protocol for a PAEROSOL application that met MAMC requirements. The final protocol provided a $3-5 \log _{10}$ per coupon reduction (to the detection limit) in viable microbial cells and spores, which were initially inoculated on the coupons of tile, countertop (Formica), and fabric. On coupons of carpet, the log reduction depended on the microbe type and was in the range, $1-4 \log _{10}$. The most effective protocol required the following:

1. PAEROSOL generation inside the test room over 30 minutes from $2.0 \mathrm{~L}$ of neutral $\mathrm{pH}$ solution of $2.5-5 \mathrm{~g} / \mathrm{L}$ table salt that has been electrochemically activated

2. The test-room remaining vacant for 4.0 hours total, including 30 minutes of PAEROSOL generation.

No additional safety precautions were taken other than shutting off the air handing system and closing the door.

The final protocol required little manpower, left no waste other than micrograms of easily wiped-off salt on horizontal surfaces, and posed no risk to those running the trial or the building occupants. No visible deterioration was observed on the furniture and electronics exposed to PAEROSOL daily, or sometimes twice a day, during the months of testing. All electronic devices remained fully operational throughout the trial. 



\section{Acknowledgments}

COL David E. McCune, MD

Chief, Department of Clinical Investigation

Consultant to the Surgeon General, Hematology/Oncology

Madigan Army Medical Center

COL James E. Cook, MD

Chief, Department of Preventive Medicine

Madigan Army Medical Center

MAJ Michele A. Soltis, MD

Associate Director, Public Health Residency Program

Chief, Epidemiology and Disease Control Service

Department of Preventive Medicine

Madigan Army Medical Center

CPT Samandra Demons, PhD

Chief, Research Microbiology

Department of Pathology

Madigan Army Medical Center

Special thanks to Joseph H. Weston, Emergency Management Planner (Madigan Healthcare System / MCHJ-CSO-E) for his unfailing assistance through the trial. Also, special thanks to the staff from the laboratory of microbiology in the Department of Pathology, and staff from the laboratory of microbiology at Department of Clinical Investigation, for their support. 



\section{Acronyms and Abbreviations}

ASTM

AOAC

$\mathrm{BD}$

BSC

$\mathrm{CDC}$

CFU

DCI

DTRA

DTRA IST

ECA

FAC

FEM

HAI

HPV

MAMC

MDR

MDRB

MRSA

MSSA

ORP

psi

$\mathrm{RH}$

ROS

PBS

SARS

TSA

TSB

VAG

VHP

VRE
American Society for Testing and Materials

Association of Official Agricultural Chemists

Becton, Dickinson and Company

biological safety cabinet

Centers for Disease Control and Prevention

colony-forming unit

MAMC Department of Clinical Investigation Animal Surgical Suite

Defense Threat Reduction Agency

Defense Threat Reduction Agency Office of Innovation Science and Technology

electrochemical activation

free available chlorine

flow-through electrochemical module

healthcare-associated infection

hydrogen peroxide vapors

Madigan Army Medical Center on Joint Base Lewis-McChord in

Tacoma, Washington

multidrug resistance

multidrug-resistant bacteria

methicillin-resistant Staphylococcus aureus

methicillin-susceptible Staphylococcus aureus

oxidation-reduction potential

pounds per square inch

relative humidity

reactive oxygen species

phosphate buffer saline

severe acute respiratory syndrome

tryptic soy agar

trypt soy broth

vortical aerosol generator

vapor hydrogen peroxide

vancomycin resistant Escherichia coli 



\section{Contents}

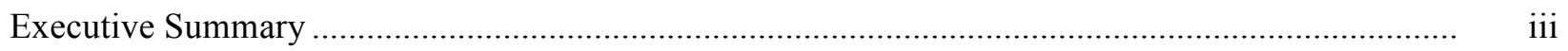

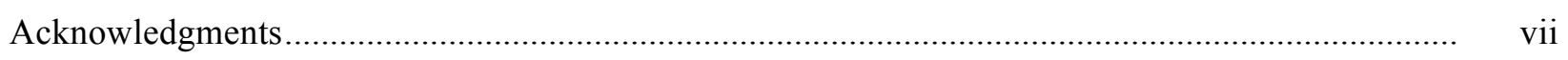

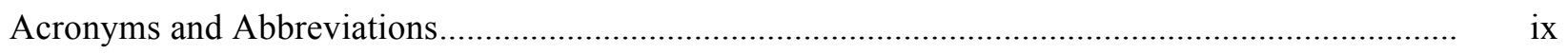

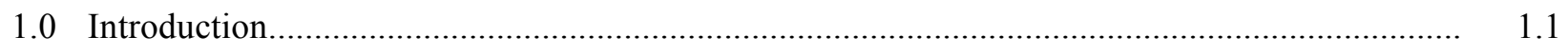

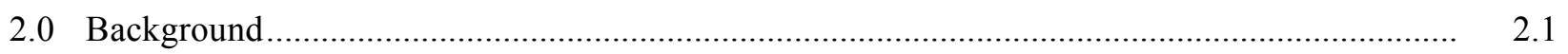

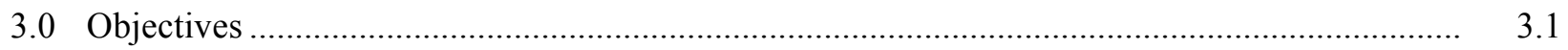

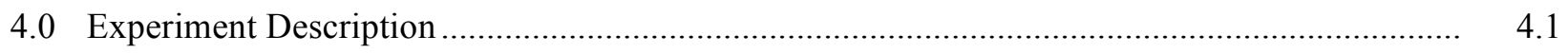

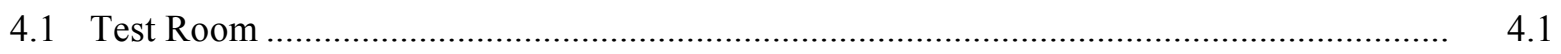

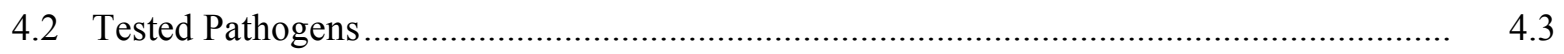

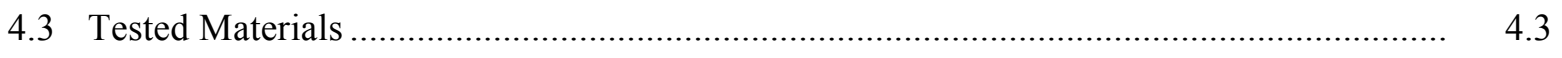

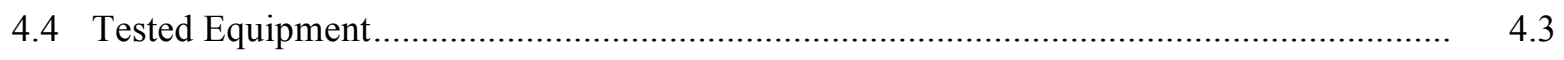

4.4.1 STEL Electrolyzer Model 10N-120-01 ............................................................ 4.3

4.4.2 Proprietary Vortical Aerosol Generator …......................................................... 4.3

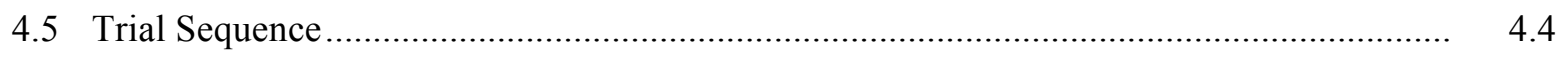

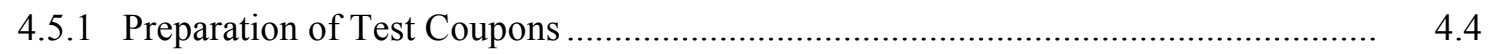

4.5.2 Preparation of Microbial Cells and Spores......................................................... 4.5

4.5.3 Spiking Test Coupons with Cells or Spores .......................................................... 4.5

4.5.4 Recovery of Cells and Spores from the Test Coupons............................................ 4.6

4.5.5 Preparation of a Liquid Disinfectant for PAEROSOL Test .................................... 4.7

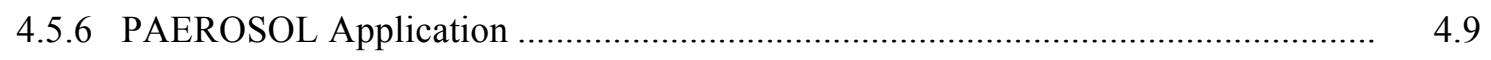

4.5.7 PAEROSOL Efficacy Assessment.................................................................... 4.10

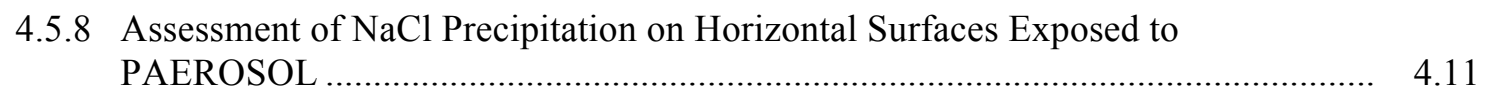

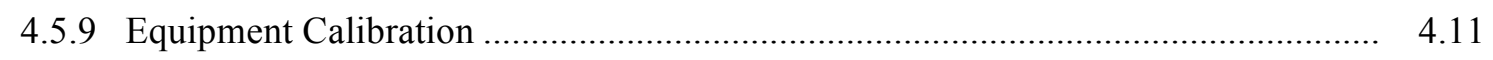

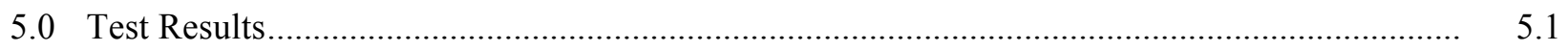

5.1 Efficacy of Microorganisms' Recovery from Contaminated Materials ............................. 5.1

5.2 Natural Decline in Viability in the Test Room Environment in the DCI at MAMCpositive Control Test (Coupons Contaminated but not Exposed to PAEROSOL) .............. 5.3

5.3 Evaluation and Optimization of PAEROSOL Application ................................................ 5.6

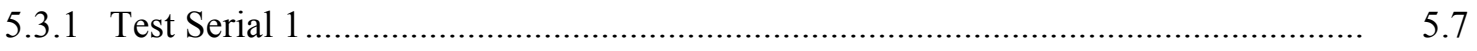

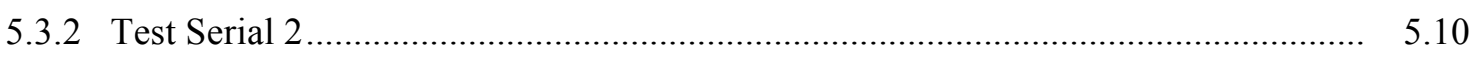

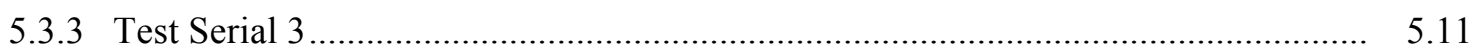

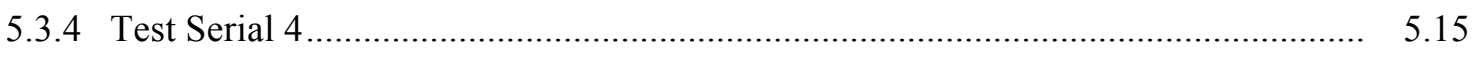

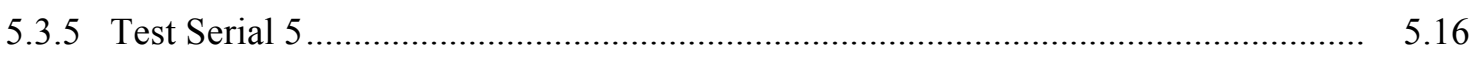

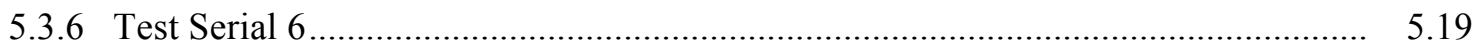

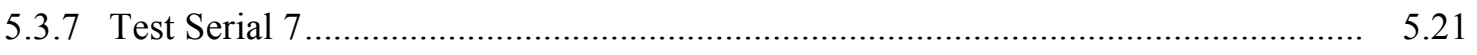

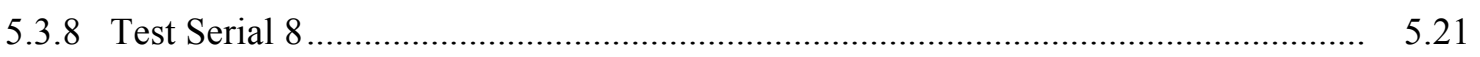




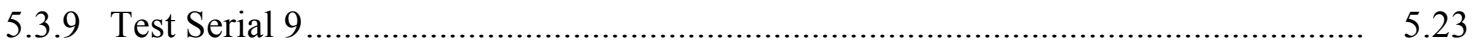

5.3.10 Damage to Coupons, Inanimate Surfaces and Test Room Equipment.................... 5.24

5.3.11 Observations and Ease of Use ........................................................................... 5.25

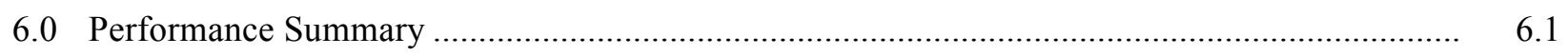

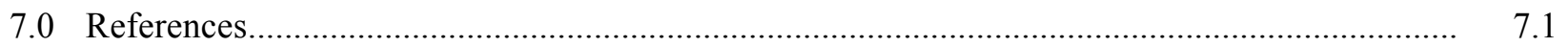




\section{Figures}

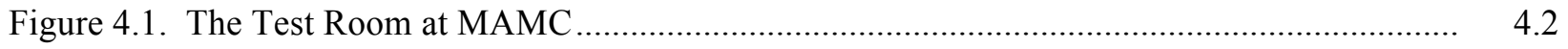

Figure 4.2. Schematic of the Test Room with Designated Areas for Coupon Placement .................. 4.2

Figure 4.3. Examples of Designated Areas in the Test Room on Countertop and Under the Table .. 4.2

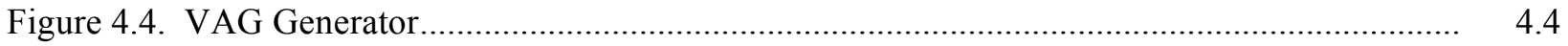

Figure 4.5. Coupon Preparation and Extraction........................................................................... 4.6

Figure 4.6. Photograph of STEL Device Connected to the Peristaltic Pump .................................... 4.7

Figure 4.7. Arrangement of the Coupons in the Test Room ........................................................ 4.9

Figure 4.8. Illustration of PAEROSOL Dispersion Inside the Test Room ....................................... 4.10

Figure 5.1. Recovery of Air-dried Microorganisms from Different Materials ................................... 5.2

Figure 5.2. K. pneumoniae Survival on Different Materials in the Control Experiment................... 5.4

Figure 5.3. S. auerous Survival on Different Materials in the Control Experiment .......................... 5.5

Figure 5.4. B. subtilis Survival on Different Materials in the Control Experiment .......................... 5.5

Figure 5.5. P aeruginosa Survival on Different Materials in the Control Experiment...................... 5.6

Figure 5.6. PAEROSOL Performance During 24 hours after Fumigation of $700 \mathrm{ml}$ of Anolyte ....... $\quad 5.8$

Figure 5.7. Percent Reduction for each Microorganism on each Material in Position 1 ................... 5.8

Figure 5.8. Percent Reduction for each Microorganism on each Material in Position 2 .................. 5.9

Figure 5.9. Percent Reduction for each Microorganism on each Material in Position 3 .................. 5.9

Figure 5.10. Kinetics of In-room RH Change after PAEROSOL Dispersion .................................. 5.10

Figure 5.11. PAEROSOL Performance During Four Hours after Dispersion of $1340 \mathrm{ml}$ of Anolyte

Figure 5.12. PAEROSOL Performance During Two Consecutive Dispersals of $1 \mathrm{~L}$ of Anolyte, with a 2-hour Break in Between ........................................................................................ 5.12

Figure 5.13. Percent Reduction for each Microorganism on each Material in Position 1 ................ 5.13

Figure 5.14. Percent Reduction for each Microorganism on each Material in Position 2 ................. 5.13

Figure 5.15. Percent Reduction for each Microorganism on each Material in Position 3 ................. 5.13

Figure 5.16. Kinetics of In-room RH Change after PAEROSOL Dispersion .................................. 5.14

Figure 5.17. PAEROSOL Performance During Two Consecutive Dispersals of $0.8 \mathrm{~L}+0.4 \mathrm{~L}$ of Anolyte with a 2-hour Break in Between

Figure 5.18. PAEROSOL Performance During Two Consecutive Dispersals of 1.0L + 1.0L of Anolyte (from $0.25 \%$ of $\mathrm{NaCl}$ ) with 2 hours Break in Between ........................................... 5.16

Figure 5.19. Percent Reduction for each Microorganism on each Material in Position 1 ................. 5.17

Figure 5.20. Percent Reduction for each Microorganism on each Material in Position 2 ................. 5.17

Figure 5.21. Percent Reduction for each Microorganism on each Material in Position 3 ................. 5.17

Figure 5.22. PAEROSOL Performance During Two Consecutive Dispersals of 1.0L + 01.0L of Anolyte (from $0.125 \% \mathrm{NaCl}$ ) with 2 Hours Break in Between ............................................... 5.18

Figure 5.23. Organisms' Reoccurrence on Tile after PAEROSOL Exposure (Decontamination

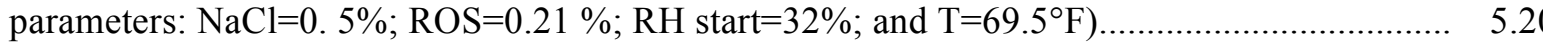


Figure 5.24. Organisms' Reoccurrence on Cloth after PAEROSOL Exposure (Decontamination parameters: $\mathrm{NaCl}=0.5 \%$; ROS $=0.21 \%$; $\mathrm{RH}$ start $=32 \%$; and $\mathrm{T}=69.5^{\circ} \mathrm{F}$ ).

Figure 5.25. Organisms' Reoccurrence on Formica after PAEROSOL Exposure

(Decontamination parameters: $\mathrm{NaCl}=0.5 \%$; $\mathrm{ROS}=0.21 \%$; $\mathrm{RH}$ start $=32 \%$; and $\mathrm{T}=69.5^{\circ} \mathrm{F}$ )......

Figure 5.26. Organisms' Reoccurrence on Carpet after PAEROSOL Exposure (Decontamination parameters: $\mathrm{NaCl}=0.5 \%$; $\mathrm{ROS}=0.21 \%$; $\mathrm{RH}$ start $=32 \%$; and $\left.\mathrm{T}=69.5^{\circ} \mathrm{F}\right)$.

Figure 5.27. $\mathrm{NaCl}$ Collected in the Test Room after PAEROSOL Fumigation

Figure 5.28. K. pneumoniae (left) and S. aureus (right) in Petri Dishes Covered with Lids During 4 hours of PAEROSOL Treatment

Figure 5.29. K. pneumoniae (Inoculated on TSA with 5\% Sheep Blood) in the Control (left) and in the Petri Dish Exposed to PAEROSOL for 4 Hours 


\section{Tables}

Table 2.1. Disinfecting Efficacy of PAEROSOL Toward Microorganisms Dried Off on Different Materials and Airborne 2.5

Table 2.2. Efficacy of PAEROSOL Produced with Different Waters .......................................... 2.6

Table 4.1. Physical Chemical Parameters of the Anolytes Produced with Different Brines .......... 4.8

Table 4.2. Physical-chemical Parameters of Diluted Anolytes.................................................... 4.8

Table 5.1. Efficacy of Microorganisms' Recovery from Contaminated Materials ${ }^{\mathrm{a}}$........................5.3

Table 5.2. $\mathrm{NaCl}$ Deposited Compared to $\mathrm{NaCl}$ Used in the Anolyte.......................................... 5.22 



\subsection{Introduction}

Infections in humans involve an exposure of a susceptible host to an infecting organism in an appropriate environment. Confined environments where large heterogeneous groups of people are crowded together-healthcare settings, college dormitories or military barracks, schools, nursing homes, cruise ships, and aircraft - serve as reservoirs for a variety of microorganisms. Therefore, an enclosed environment is often the risk factor that leads to the acquisition of the infection. In 2007, schools across the country reported outbreaks of staph infections that forced districts to call off classes, cancel sporting events, and disinfect entire buildings (Urbina 2007). On-board influenza transmission and the emergence of severe acute respiratory syndrome (SARS) in 2003 demonstrated the potential of a disease to spread globally in the air (European Centre for Disease Prevention and Control 2010; Wilder-Smith and Leong 2004). A comprehensive review of infections transmission in cruise ships, military barracks, and college dormitories was published by Kak (2007).

Whether people exposed to infective material then develop disease depends on the dose they receive and their general state of health. If the body's defenses are weakened by infection, disease, surgery, chemotherapy, or natural aging, the body is more susceptible to further infection. In this regard, the hospitals represent unique confined environments where the majority of occupants (patients) are ill to higher or lesser degree, and by definition, their intrinsic immune defense is significantly reduced. Additionally, there are vast number of patients with chronic diseases and elderly people in the hospitals and nursing homes whose immune systems are seriously compromised. All these factors make healthcare settings the most vulnerable enclosed environment where the occupants are at highest risk of crosscontamination, compared to the other confined environments.

Nosocomial, or healthcare-associated infections (HAIs), are among the leading causes of death in the United States. An HAI is defined as an infection developing in hospitalized patients, neither present nor in incubation at the time of their admission. The Centers for Disease Control and Prevention (CDC) estimate that in the United States, as many as one in ten patients, or approximately 2 million patients a year, acquire a nosocomial infection. Of those patients, about 90,000 die as a result of an HAI. Estimates of the resulting annual cost range from $\$ 4.5$ billion to $\$ 11$ billion. Therefore, in today's healthcare setting, minimizing a patient's risk of HAI is of great concern (Centers for Disease Control 2002)

Klebsiella pneumoniae, methicillin-resistant Staphylococcus aureus (MRSA), methicillin-susceptible Staphylococcus aureus (MSSA), vancomycin resistant Escherichia coli (VRE), Acinetobacter spp, Clostridium difficile, Proteus spp, and Pseudomonas aeruginosa are among the most common causative agents of HAIs, and all of them are prone to multidrug resistance (MDR). Multidrug-resistant bacteria (MDRB) are often difficult to treat because of their innate or acquired resistance to multiple classes of antimicrobial agents. A primary reason for concern about these MDRB is that options for treating patients with these infections are often extremely limited, if any, and MDR infections are associated with increased lengths of stay, costs, and increased mortality. MDRB have emerged as a public health problem. Extensive use of broad-spectrum antibiotics in general, and particularly for controlling HAIs in the hospital environment, significantly contributes to MDRB appearance and promotes emergence of newer antibiotic-resistant organisms (Jain and Singh 2007; Flanders et al. 2006).

The roles of medical devices (such as catheters and stethoscopes) and personnel and patient hygiene in the transmission of HAIs have long been recognized. However, the role of hospital inanimate surfaces 
in the transmission of HAIs has been unclear for a long time. For the last decade, numerous studies have shown that hospital inanimate surfaces, including medical equipment, are contaminated by a variety of microorganisms. The pathogens that have already been linked to transmission via contaminated surfaces, both environmental and medical equipment, include MRSA, VRE, C. difficile, Acinetobacter spp., and norovirus (Hayden et al. 2006; Carling et al. 2008a). Except for norovirus, these organisms pose clinically important antimicrobial resistance problems and are among the most common causes of HAIs in intensive care units (Hidron et al. 2008; McDonald et al. 2006).

The organisms can be expelled from infected or colonized patients either through direct contact, aerosol droplets, or feces. While direct contacts play an important role in cross-contamination via "high touch" surfaces in the immediate vicinity of a patient, aerosols exhaled from talking, sneezing, and coughing could be a source for air contamination and for microorganisms landing on floor, walls, privacy curtains, window sills, and other inanimate surfaces not considered "high touch" (Wainwright et al. 2009; Carvalho et al. 2007). Talking for 5 minutes and coughing each produce 3,000 droplet nuclei, and sneezing can generate approximately 40,000 droplets, which then evaporate to particles in the size range of $0.5-12 \mu \mathrm{m}$. If the droplets are large enough, bioaerosols can fall to surfaces because of gravity. Droplet nuclei of $2 \mu \mathrm{m}$ will take 4.2 hours to fall 6 feet and can remain suspended in the air for several hours and travel over long distances with airflow before being deposited on surfaces or mucous membranes (Memarzadeh 2012).

A team from Yale University recently reported that a person's mere presence in a room can add 37 million bacteria to the air every hour - material largely left behind by previous occupants and stirred up from the floor. The team also claimed that human microbiota in airborne particulate matter in an occupied setting demonstrated that the aerosol route can be a source of exposure to microorganisms emitted from the skin, hair, nostrils, and mouths of other occupants (Qian et al. 2012). Environmental contamination through aerosols produced by high-speed cutters in lumbar spine surgery was confirmed in the study performed by Nogler et al. (2001). Rautemaa et al. (2006) demonstrated that high-speed rotating instruments in restorative dentistry create aerosols containing microbes from the oral cavity of the patients, and these aerosols spread microbes across the room at all distances. Moreover, the contamination level was found to be higher in the more remote sampling points.

Being deposited on inanimate surfaces, common HAI pathogens were shown to be quite resistant to the hospital environment. With a survival time from a few days to many months, these pathogens remained a viable source of cross-contamination (Kramer et al. 2006; Sexton et al. 2006; Huang et al. 2006).

Manual cleaning and disinfecting that have been adopted in healthcare settings worldwide are very challenging procedures. While seemingly simple, manual cleaning is very laborious, and requires a welltrained staff, and significant time and effort for a cleaning crew to achieve a desirable level of disinfection. Manual cleaning focuses on "high-touch" surfaces in patient-care areas, but the HAI pathogens were found on walls, furniture, windowsills, and chairs. Because they are deposited on the surfaces, HAI pathogens could be re-aerosolized by in-room activities, including room cleaning and ventilation. It was shown that routine bedmaking prompted the appearance of MRSA in the air, followed by re-deposition of the pathogens on the various surfaces inside the room (Shiomori et al. 2002; Carvalho et al. 2007). Air-borne dissemination of microbes from flushing a toilet resulted in contamination of surfaces in the toilet cubicle and beyond (Barker and Jones 2005). 
Recent studies have focused on evaluating cleaning protocols, actually measuring the thoroughness of cleaning to assess the effectiveness of its use as a basic step in ensuring the removal of potential pathogens. Hundreds of hospital wards and many thousands of "high touch" surfaces were examined. High to moderate rates of cleaning of traditional sites, but poor cleaning of many sites that have significant potential for harboring and transmitting microbial pathogens, was demonstrated. The overall thoroughness of terminal cleaning, expressed as a percentage of "high touch" surfaces examined, was 45 to 49 percent. The frequency with which various individual sites were cleaned varied widely but was similar in all hospitals (Carling 2006; Carling 2008a; Carling 2008b). Inadequate cleaning of surfaces has been shown to actually increase the sporulation of $C$. difficile and to increase its spread during the manual cleaning process (Wilcox and Fawley 2000).

It is a fact that the efficacy of manual cleaning and disinfecting protocols closely depends on human factors and on cleaning/bactericidal chemicals. Environmental workers are usually the least-educated, lowest-paid personnel, and have very high turnover rates. Therefore, improving cleaning crew skills is not often achievable. Efficacy of germicides applied in healthcare settings and growing HAI resistance to conventional germicides also contribute to unsatisfactory efficacy of HAIs disinfection. The relationship between microbial resistance to antibiotics and germicides is still being debated; however, regular rotation of disinfectants to prevent an appearance of disinfectant-resistant pathogens is required in healthcare settings (Maillard 2005).

The revelation that manual disinfecting in hospitals has significant drawbacks and that HAI pathogens, including drug-resistant organisms, were recovered from inanimate surfaces even after terminal cleaning, has brought about an awareness of the necessity for new disinfecting methods and protocols for healthcare settings. There has long been interest in disinfecting healthcare settings with a universal fumigant that is capable of eradicating all HAI pathogens in the air, and from all room surfaces, including those surfaces that are difficult to access. In the 1960s, in addition to the standard terminal cleaning, the use of the spray-fog technique was a relatively common method for decontaminating patient rooms. In this practice, quaternary ammonium compounds, phenolics, paraformaldehyde, and/or hypochlorite solutions were dispersed into sealed hospital rooms by a fog generator placed in the room. In 1972, the CDC published a review that concluded that fogging had no role in the terminal disinfection of hospital room (Centers for Disease Control 1972). Subsequently, the U.S. hospitals eliminated the use of fogging, and concern about decontaminating environmental surfaces abated.

Because of new evidence that contaminated environmental surfaces contribute to the transmission of HAIs (Drees et al. 2006; Hayden et al. 2006; Huang et al. 2006) greater attention is now being given to the methods that could clean the surfaces beyond "high touch" areas (Boyce 2009). The contamination of the U.S. government buildings with the anthrax bacillus in 2001 also generated new interest in the methods for decontaminating rooms and other large spaces in buildings. Vapor-phase fumigants, including formaldehyde, chlorine dioxide $\left(\mathrm{ClO}_{2}\right)$, and hydrogen peroxide, were considered to eliminate Bacillus anthracis from government buildings. In spite of well-proven formaldehyde efficacy toward Bacillus spores, its use in building remediation and sensitive equipment decontamination was concluded as undesirable because of its serious health, environmental, and operational disadvantages (McAnoy 2006). Although chlorine dioxide was shown highly effective and was extensively used in the decontamination of government buildings, multiple environmental, health, operational, and logistics complexities were observed that require further study and modifications (McAnoy 2006). 
The use of hydrogen peroxide vapor decontamination has gained considerable interest in defense (and other industries) as a wide ranging decontaminant for use in multiple scenarios and against multiple biological and chemical contaminants. The technology has already been used successfully in real building remediation scenarios following the 2001 anthrax attacks in the United States (EPA 2005). Although effective, an application of hydrogen peroxide vapors also required strong isolation/insulations of the decontaminating environment because of the peroxide toxicity and the operational necessity to control special environmental conditions in decontaminating environment to support peroxide vapors efficacy.

In spite of recognized logistics and operational drawbacks, ozone, $\mathrm{ClO}_{2}$, and specifically hydrogen peroxide vapors have been studied broadly over the years to promote their application in hospitals. However, the transition of logistically complex disinfection technologies from the field of threat reduction to healthcare settings was not a simple task. While strict isolation/insulation of the entire environment contaminated with bio-threat agents is imperative to exclude further dissemination of highly dangerous pathogens, routine disinfection of hospital facilities takes place in the midst of a busy environment where the necessity of rigorous insulation of each ward or surgery room becomes a real burden for hospital management.

Ozone was shown effective against Gram-negative and Gram-positive bacteria, molds, and bacterial spores (Sharma and Hudson 2008; de Boer et al. 2006; Moat et al. 2009). However, because of its toxicity and environmental impact, ozone has been investigated in the hospital environment on a limited basis and the results were not consistent (Berrington and Pedler 1998). Recently, the combination of ozone with hydrogen peroxide vapor was examined in coupon-based chamber-scale experiments, and in an isolated laboratory room. $A \geq 6 \log _{10}$ reduction in the bacteria and spores was achieved with HAI pathogens. However, to use an ozone-peroxide system within the occupied laboratory building, the entire test-room except for the cement floor was sealed with 3-mil polyethylene plastic sheeting and polypropylene tape to make it airtight. During experimental runs, the door was taped shut and the room's air-handling system was turned off to protect against ozone-peroxide gas spread outside the room while a fan inside the room was needed to provide disinfectant distribution in the room (Zoutman et al. 2011).

Gaseous $\mathrm{ClO}_{2}$ has been shown to be an effective disinfectant especially related to the use in the medical science areas. Rosenblatt et al. developed the use of gaseous $\mathrm{ClO}_{2}$ to sterilize gas-impermeable surfaces of implements commonly employed in medical sciences, such as those made of porcelain, ceramics, metal, plastics, and glass (Rosenblatt et al. 1985; Rosenblatt et al. 1987). Jeng and Woodworth (1990) reported the sporicidal activity of $\mathrm{ClO}_{2}$ gas under square-wave conditions within an experimental sterilizer used for medical implements. Luftman et al. (2004) reported very successful $\mathrm{ClO}_{2}$ application in large animal intensive care and neonatal intensive care units contaminated with Salmonella newport. Evidently, $\mathrm{ClO}_{2}$ is very potent disinfectant; however, its toxicity, necessary insolation of decontaminating arena, and strong requirements to support certain humidity during entire disinfecting makes its application difficult for hospital routine protocols. Chlorine dioxide-based disinfecting of a hospital contaminated with mold required removing the entire contents of the hospital before the building was covered with a large tarpaulin and then fumigated for 24 hours with chlorine dioxide, at a cost of \$25 million (Davies et al. 2011).

Gaseous hydrogen peroxide is the most extensively studied fumigant during the last 30 years. The high efficacy of hydrogen peroxide vapors (HPV) on practically all important HAI pathogens was confirmed in laboratory conditions, in hospital settings, in food processing, and pharmaceutical industries 
(Bioquell 2011). HPV is generally regarded as less toxic than many other gaseous decontaminants, since it breaks down to water and oxygen. Over the years, many variations have been applied to the HPVbased decontamination process. Currently, there are two commercially available methods for using hydrogen peroxide vapor as a disinfectant: HPV by Bioquell (2012) and vapor hydrogen peroxide (VHP) by STERIS (STERIS 2012). Both systems have been shown to be effective (Fisher and Caputo 2004).

Although hydrogen peroxide is proposed as the most suitable and effective gaseous decontamination technology, it has significant limitations for use in hospital environments. The limitations include strong requirements for thorough room-pre-cleaning, facility insulation, well-trained personnel, and expensive equipment that require maintenance and control. Additionally, compatibility of VHP and HPV with various interior materials and electronics remains questionable. The Showcase Hospital Report on the VHP system application was published in 2009 (Department of Health/NHS Purchasing and Supply Agency) and reported,

"Here was no clear evidence linking reduced levels of environmental contamination to lower levels of infection, though it was a reasonable expectation that there should be some effect, and there was some limited evidence, particularly in relation to $\mathrm{C}$. difficile, that HPV disinfection through elimination of the spores, may lower subsequent incidence of disease."

Whether or not HPV and VHP decontamination is feasible in busy United States hospitals for decontamination of routine rooms, or at least for terminal disinfection after a patient is discharged, remains a big question. The Showcase Hospital Report (Department of Health/NHS Purchasing and Supply Agency 2009) states,

"Best value can be derived from the Bioquell system by placing items of equipment, which have been identified as needing disinfection, such as electronic equipment, drip stands, commodes and wheelchairs, in rooms which need to be disinfected."

This recommendation speaks for itself.

Although, VHP and HPV, and in lesser degree $\mathrm{ClO}_{2}$, might be useful to combat serious infections outbreaks within large confined environment, their utilization for routine control of HAIs in healthcare settings is not practical, yet. The application of these technologies is too complex and expensive for incorporation into the daily routine to reduce the risk of cross-contamination. Although these methods could be implemented in crisis scenarios, such as significant outbreaks, they are not appropriate to prevent outbreaks in the hospitals. At the same time, because of the multifactorial nature of HAIs, the tendency to affect immunosuppressed patients, and often multi-drug resistant organisms, the primary method to avoid morbidity/mortality and costs associated with these infections is through prevention.

In general, gas or vapor provides the type of disinfection that increases the probability of an effective reduction of viable pathogens in the air and on inanimate surfaces. However, a new approach needs to be developed around this delivery method. To be practical, the methods have to be effective against various pathogens, be compatible with interior materials and electronics, be environmentally safe, have minimal impact on logistics and manpower, and have low cost. 
The Defense Threat Reduction Agency Office of Innovation Science and Technology (DTRA IST) evaluated a new disinfecting approach entitled PAEROSOL that has been developed by the U.S. Department of Energy's Pacific Northwest National Laboratory (PNNL) in collaboration and cooperation with the Institute of Highly Pure Bio-Preparation and the World Health Organization (WHO) Center "Institute of Influenza" (in St. Petersburg, Russia). Gas-like PAEROSOL decontamination is based on atomized electrochemically energized solutions of table salt. It was previously shown to be highly effective in the eradication of $\geq 6 \log _{10}\left(10 \log _{10} / \mathrm{m}^{2}\right)$ microbial and viral pathogens, spores, and mold on various interior materials and in the air. PAEROSOL is also compatible with electronic equipment, not toxic to warm-blooded animals, simple to use, and inexpensive (Sventitskyi et al. 2011). DTRA IST sponsored PNNL to perform a series of studies in the Madigan Army Medical Center (MAMC) on Joint Base Lewis-McChord in Tacoma, Washington, to validate the previously observed high efficacy of PAEROSOL bactericidal performance in the U.S. hospital setting. 


\subsection{Background}

PAEROSOL is a semi-dry microaerosol composed of anolyte that has been produced by electrochemical activation of 0.25 to 0.5 percent table salt.

Electrochemical activation (ECA) technology as a specialized discipline of electrochemistry was developed in the 1970s in the former Soviet Union (Bakhir 2012). The electrochemical activation is a combination of electrochemical and electro-physical exposure of water-containing ions and molecules of dissolved substances. The electrochemical process occurs in the area of spatial charge close to either the anode or cathode of the electrochemical system in conditions of non-equilibrium charge transferred by electrons through the "electrode-electrolyte" interface. Water activated near the cathode (catholyte) possesses increased electron activity and has well-pronounced reductant properties. Correspondingly, water activated near the anode (anolyte) is characterized by inhibited electron activity and demonstrates oxidant properties. Electrochemical activation enables the synthesis of chemical reagents (oxidants and reductant) from water and substances dissolved in it, to a metastable state (changing readily and having more free energy). Electrochemically activated solutions are generated by passing a dilute salt solution through an electric field in a flow-through electrochemical module (FEM), separating the ions formed, and producing two oppositely charged solutions possessing altered physical and chemical properties (Bakhir and Zadorozhny 1997). After electrochemical activation, ECA solutions possess gradually transitioning physical-chemical properties (parameters) from a metastable state into a state of thermodynamic equilibrium.

An ECA negatively charged catholyte possesses very interesting properties as a degreasing/cleaning agent (Marais 2000; Marais and Brozel 1999; Marais and Williams 2001; Solovyeva and Dummer 2000), but it is not bactericidal, and therefore it will not be discussed here.

The positively charged solution (anolyte) has outstanding bactericidal property, typically has a redox value of $>+900 \mathrm{mV}$, and is composed of a mixture of unstable oxidants in a physically excited state. In accordance with Bakhir et al. (2003) oxygen-chlorine compounds resulting from electrochemical activation have the highest bactericidal activity in the range of $\mathrm{pH}$ from 7.0 to 7.6, where concentrations of hypochlorite-ions and hypochlorous acid are close to each other. Bakhir et al. (2003) explained it: the chemicals mentioned are conjugate acid and base

$$
\left(\mathrm{HClO}+\mathrm{H}_{2} \mathrm{O}{ }^{\circledR} \mathrm{H}_{3} \mathrm{O}^{+}+\mathrm{ClO}^{-} ; \mathrm{ClO}^{-}+\mathrm{H}_{2} \mathrm{O}{ }^{\circledR} \mathrm{HClO}+\mathrm{OH}^{-}\right)
$$

and they form a metastable system, which can generate further active components that possess higher than hypochlorous acid biocidal efficiency:

- ${ }^{1} \mathrm{O}_{2}$ - singlet molecular oxygen

- $\mathrm{ClO}$ - hypochlorite-radical

- $\mathrm{Cl}$ - chlorine-radical (atomic chlorine)

- $\mathrm{O}$ - atomic oxygen

- $\mathrm{OH}$ - hydroxyl-radical

- $\mathrm{O}_{3-\text { ozone }}$ 
- $\mathrm{H}_{2} \mathrm{O}_{2}$ - hydrogen peroxide

- $\mathrm{ClO}_{2}-$ chlorine dioxide.

$\mathrm{H}^{+}$and $\mathrm{OH}^{-}$are the catalysts of reactions with participation of oxi-chlorine ions and radicals. Concentrations of $\mathrm{H}^{+}$and $\mathrm{OH}^{-}$ions are similar in water at a $\mathrm{pH}$ close to neutral. The variety and metastable concentration of reactive oxygen species (ROS) in anolytes depends on $\mathrm{pH}$ of the resultant anolyte, the module used for electrochemical activation, concentration of sodium chloride in brine, current, voltage, electrolyzer engineering design, and possibly other factors that have not yet been observed. In all bactericidal anolytes, free available chlorine (FAC) is the primary analyzable oxidant constituent. A major problem to determining additional oxidant species present in anolytes is the lack of analytical techniques for differentiating oxidants. Oxidant analyses for $\mathrm{FAC}, \mathrm{O}_{3}, \mathrm{H}_{2} \mathrm{O}_{2}$ and $\mathrm{ClO}_{2}$ are continuingly improving. Almost invariably, each of the methods is based on the total oxidizing capacity of the solution being analyzed and is readily subject to interferences from the presence of other potential oxidizing agents or intermediates from associated chemical reactions. This is especially the case for analyzing additional oxidants in the presence of free chlorine. Though theoretically there could be various $\mathrm{ROS}$ in anolytes at $\mathrm{pH}$ 7, practically neither the variety of ROS nor their real concentrations in anolytes are known because of the imperfection of current analytical tool and because of its intrinsic metastability. Recent research has provided evidence that stronger-than-chlorine oxidants are present in the anolytes. In addition to the hypochlorous acid and hypochlorite ion, $\mathrm{O}_{3}, \mathrm{H}_{2} \mathrm{O}_{2}$, and hydroxyl free radicals $(\mathrm{HO} \bullet$,$) were detected at the point of generation (MIOX 2011; Jeong et al. 2006).$

During relaxation time (the metastable solution relaxes back to the absolute energy minimum), characteristics of anolyte are gradually changing as compared to the initial (immediately after electrochemical/electro-physical exposure) value, and bactericidal efficacy of the anolyte is reduced. The longevity of anolyte relaxation time (so-called anolyte bactericidal stability) analyzed in different laboratories ranges from 48-72 hours (Prilutsky and Bakhir 1997) to 7 weeks (Grosser 2001) to 1 year (Puricore 2012; Envirolyte 2012). The reason of such significant difference is not yet understood.

Previous studies have recognized that various ECA anolytes are easily produced, environmentally safe, and non-corrosive super-disinfecting agents against various bacterial and viral organisms (Trustwater 2011; Aggarwal et al. 2010; Rogers et al. 2006; O'Donnell et al. 2009; Cloete et al. 2009; Venkitanarayanan et al. 1999; Deza et al. 2003; Marais and Brözel 1999; Marais and Williams 2001). The U.S. Marine Corps began testing ECA anolyte (ECASOL) as a highly effective biocidal agent solution in 1998 to assess safety, efficacy and the potential to scale-up field units for use with first or secondary response personnel (Grosser 2001). Overall, anolyte products have been tested by many institutions nationally and internationally and received regulatory approvals for application in different fields (Activated Environmental Solutions Inc. 2012)

Electrochemically activated anolytes were proved to be outstandingly effective, environmentally safe biocides that are well comparable with, or even exceeding efficacy of conventional germicides (Sigua et al. 2011). However, as a liquid germicide ECA anolyte has almost the same disadvantages for decontamination of beyond "high-touch" inanimate surfaces and air, as all other liquid disinfectants.

Despite projections for use of ECA anolytes as a fog in the 1970s (Prilutsky and Bakhir 1997), minimal results of anolyte application as fumigant were reported. In 2002, Electro-Chemical Technologies Ltd. announced that the U.S. Department of Defense began testing the fog forms of its 
biological decontaminant known as ECASOL (The Free Library by Farlex 2012). However, no results of that testing became publicly available. The first results on super-oxidized water fogging for environmental decontamination were published by Clark et al. (2006). In that study the decontamination efficacy of aerosolized particles of Sterilox pH $5.8(\varnothing 5-50 \mu \mathrm{m})$ was examined with MRSA and Acinetobacter dried on ceramic tiles positioned horizontally. The experimental set-up was specifically designed in such a way that helped aerosol droplets to settle down on the coupons and thus, to contact the bio load as liquid. A $6 \log _{10}$ reduction of $8 \log _{10}$ initial bio load was achieved for both pathogens twice exposed to Sterilox. Although, the results were positive, the study once more confirmed efficacy of liquid Sterilox "delivered" to the surfaces as fog droplets.

Park et al. (2007) performed a very similar study with Sterilox-fog for the decontamination of carriers inoculated with human norovirus, and again the efficacy of aerosolized Sterilox resulted from liquid Sterilox that eventually accumulated on contaminated materials. In this study, contaminated carriers were positioned horizontally and vertically, and it was shown that decontaminating efficacy on horizontal carriers was higher than that on vertical ones. Observed variation of the effectiveness of fogging was logically attributed to the differences in the volume of Sterilox fog that was deposited on vertical and horizontal surfaces. After Sterilox settled on the coupons, FAC concentration and $\mathrm{pH}$ were analyzed in the liquid precipitate. Interestingly enough, it was observed that fogging itself reduced the concentration of FAC in Sterilox by approximately 70 percent and increased the $\mathrm{pH}$ by about $1.3 \mathrm{pH}$ units. Loss of chlorine in oxidized water under open and agitated conditions was also demonstrated by Len et al. (2002). It was shown that in open conditions, the chlorine loss was primarily through the evaporation of dissolved chlorine gas and agitation enhanced the chlorine loss through evaporation by accelerating interface mass transfer of chlorine gas. Appreciable loss of FAC during Sterilox fogging was observed as a strong negative factor that led to limited interest in using ECA anolytes as fumigant. A similar opinion was expressed by Dr. J. Rogers (Battelle Memorial Institute) whose point was that the fumigation of ECA anolyte might result in increased outgassing of radicals and, thus decrease the microbe-killing ability of the mist. ${ }^{1}$

Contrary to the opinion that outgassing of reactive species is a negative factor that reduces anolyte bactericidal efficacy during fumigation, we have perceived reactive species offgassing from aerosol droplets as a very positive and very important factor. We have built the PAEROSOL concept upon this factor.

We have assumed that each droplet of anolyte aerosol possesses intrinsic properties of metastable liquid anolyte and acts as a micro-reactor continuingly producing highly energized mixed ROS. We hypothesized that ROS are effectively offgassing from the surface of aerosol droplets through interfacial mass transfer, as was observed for chlorine (Len et al. 2002; Park et al. 2007). Such highly energized offgassing of ROS (e.g., free radicals), reach/contact microorganisms residing on inanimate surfaces and airborne, and either destroy microorganisms "from outside" by disintegrating their outer membranes, or penetrating inside to cause oxidative damage leading to microorganism death (Cloete et al. 2009). We have also hypothesized that the diameter of aerosol particles must be of an optimal size, enough small to behave like a gas providing effective uniform distribution of disinfectant within the entire confined space; however, not too small to be dried off instantly upon generation. Favorable surface-to-volume ratio of

\footnotetext{
${ }^{1}$ Erbschloe, DR. 2010. Personal communication with Dr. Donald R Erbschloe, Chief Scientist, Air Mobility Command, Scott Air Force Base from Evguenia Rainina (Pacific Northwest National Laboratory), February 23, 2010 .
} 
such microns-size aerosol particles promotes efficient ROS offgassing. We hypothesized that ROS are offgassing from droplets until all droplets become desiccated. Overall, we hypothesized that the anolyte aerosol remains germicidal until all droplets desiccate and the bulk gas phase ROS concentration falls below a critical level.

The hypothesis has been tested and resulted in a development of PAEROSOL-a new type of decontaminating approach.

PAEROSOL is a semi-dry fog (particles $\varnothing$ in the range of $0.5-10 \mathrm{~m} \mu$ ) generated by a high output pneumatic vortical aerosol generator (VAG). PAEROSOL is composed of highly germicidal neutral anolyte $(\mathrm{pH} \sim 7.0)$ that is produced by electrochemical activation of 0.25 to 0.5 percent table salt.

PAEROSOL behaves like a gas and we demonstrated that it eventually reached all areas in confined environments of $2,000 \mathrm{ft}^{3}$ to $20,000 \mathrm{ft}^{3}$, including those that are difficult to reach.

PAEROSOL was shown capable of eradicating $10^{6} / \mathrm{cm}^{2}$ live microorganisms, including Gram-positive and Gram-negative bacteria, microbial and fungal spores, and viruses on various materials and surfaces, including the surfaces of complex geometry, and those where PAEROSOL had no direct access. The array of examined bioagents includes simulates of bio-threat agents (Anthrax simulate), civilian and military hospitals pathogens, antibiotic-resistant microbes, viruses including H1N1 (swine flu), and H5N1 (bird flu) subtypes, as well as food-, and water-borne pathogens (Table 2.1).

Because water constitutes 99.5 percent of the mass of bactericidal anolyte used in PAEROSOL, we examined the influence of different waters on PAEROSOL bactericidal efficacy. All tests were conducted in identical conditions in a chamber of $\sim 100 \mathrm{ft}^{3}$ with $B$. cereus spores dried on glass (Table 2.2). 
Table 2.1. Disinfecting Efficacy of PAEROSOL Toward Microorganisms Dried Off on Different Materials and Airborne

\begin{tabular}{|c|c|c|c|c|c|c|}
\hline Test microbe & $\begin{array}{c}\text { Materials or } \\
\text { Airborne }\end{array}$ & $\begin{array}{l}\text { Organism, } \\
\mathrm{CFU} / \mathrm{cm}^{2} \\
\mathrm{CFU} / \mathrm{M}^{3}\end{array}$ & $\begin{array}{c}\text { Exposure to } \\
\text { PAEROSOL, } \\
\text { hours }\end{array}$ & $\begin{array}{c}\text { Organism } \\
\text { survived } \\
\mathrm{CFU} / \mathrm{cm}^{2} / \mathrm{m}^{3}\end{array}$ & $\begin{array}{c}\text { Reduction } \\
\text { versus } \\
\text { inoculated, \% }\end{array}$ & $\begin{array}{c}\text { Reduction } \\
\text { versus natural } \\
\text { die off, } \%\end{array}$ \\
\hline S. aureus & $\begin{array}{c}\text { Glass, Tile, } \\
\text { Fibrous } \\
\text { cotton } \\
\end{array}$ & $3 \times 10^{6}$ & 0.5 & $<3$ & 99,9999 & 99,9999 \\
\hline$M R S A$ & $\begin{array}{l}\text { Glass, SS, } \\
\text { hospital } \\
\text { curtains }\end{array}$ & $1.5 \times 10^{6}$ & 1.0 & $<3$ & 99,9999 & 99,9999 \\
\hline S. enteritidis & $\begin{array}{l}\text { Green leafs, } \\
\text { egg shell, } \\
\text { polyethylene }\end{array}$ & $0.5 \times 10^{6}$ & 1.0 & $<1$ & 99,9999 & 99,9999 \\
\hline A. baumannii & $\begin{array}{c}\text { Hospital } \\
\text { curtains, } \\
\text { glass, SS, } \\
\text { fibrous } \\
\text { cotton }\end{array}$ & $2.4 \times 10^{6}$ & 1.5 & $<1$ & 99,9999 & 99,9999 \\
\hline E. coli & $\begin{array}{c}\text { Plastic, } \\
\text { glass, brick, } \\
\text { latex wood }\end{array}$ & $3 \times 10^{6}$ & 0.5 & $<1$ & 99,9999 & 99,9999 \\
\hline M. tuberculosis & \multirow{2}{*}{$\begin{array}{l}\text { Tile, oilcloth } \\
\text { both sides, } \\
\text { cotton }\end{array}$} & $2 \times 10^{4}$ & 10 & $<3$ & 99,99 & 99,99 \\
\hline $\begin{array}{c}\text { M. tuberculosis } \\
\text { MDR }\end{array}$ & & $1.5 \times 10^{4}$ & 10 & $<3$ & 99,99 & 99,99 \\
\hline S. aureus & AIRBORNE & $10^{6}$ & 0.2 & $<1$ & 99,9999 & 99,9999 \\
\hline MRSA & AIRBORNE & $10^{6}$ & 0.3 & $<1$ & 99,9999 & 99,9999 \\
\hline S. enteritidis & AIRBORNE & $10^{6}$ & 0.5 & $<1$ & 99,9999 & 99,9999 \\
\hline A. baumannii & AIRBORNE & $10^{6}$ & 0.5 & $<1$ & 99,9999 & 99,9999 \\
\hline E. coli & AIRBORNE & $10^{6}$ & 0.2 & $<1$ & 99,9999 & 99,9999 \\
\hline $\begin{array}{l}\text { B. cereus } \\
\text { Spores }\end{array}$ & \multirow{2}{*}{$\begin{array}{c}\text { Fibrous } \\
\text { cotton, tile, } \\
\text { glass }\end{array}$} & $10^{6}$ & $10-14$ & $<1$ & 99,9999 & 99,9999 \\
\hline $\begin{array}{l}\text { B.thuringiensis } \\
\text { Spores }\end{array}$ & & $10^{6}$ & $10-14$ & $<1$ & 99,9999 & 99,9999 \\
\hline $\begin{array}{c}\text { B. cereus } \\
\text { Spores }\end{array}$ & AIRBORNE & $10^{6}$ & 0.3 & $<1$ & 99,9999 & 99,9999 \\
\hline $\begin{array}{l}\text { B.thuringiensis } \\
\text { Spores }\end{array}$ & AIRBORNE & $10^{6}$ & 0.3 & $<1$ & 99,9999 & 99,9999 \\
\hline $\begin{array}{c}\text { H1N1 A/Puerto } \\
\text { Rico/8/34 } \\
\end{array}$ & \multirow{2}{*}{$\begin{array}{l}\text { Glass, } \\
\text { fibrous } \\
\text { cotton, tile }\end{array}$} & $10^{4} \mathrm{EID}_{50} / \mathrm{cm}^{2}$ & 0.5 & $<1$ & 99,99 & 99,99 \\
\hline $\begin{array}{c}\text { H5N1a/Duck/ } \\
\text { Kurgan/5/05 }\end{array}$ & & $10^{4} \mathrm{EID}_{50} / \mathrm{cm}^{2}$ & 0.5 & $<1$ & 99,99 & 99,99 \\
\hline $\begin{array}{l}\text { H1N1A/ Puerto } \\
\text { Rico/8/34 }\end{array}$ & AIRBORNE & $10^{6} \mathrm{EID}_{50} / \mathrm{m}^{3}$ & 0.1 & $<1$ & 99,9999 & 99,9999 \\
\hline $\begin{array}{l}\text { H5N1a/Duck/ } \\
\text { Kurgan/5/05 }\end{array}$ & AIRBORNE & $10^{6} \mathrm{EID}_{50} / \mathrm{m}^{3}$ & 0.1 & $<1$ & 99,9999 & 99,9999 \\
\hline
\end{tabular}


Table 2.2. Efficacy of PAEROSOL Produced with Different Waters

\begin{tabular}{|l|l|c|c|c|c|}
\hline \multicolumn{2}{|c|}{ Water } & pH & FAC, \% & \multirow{2}{*}{$\begin{array}{c}\text { B. cereus } \text { dried on } \\
\text { glass ,CFU/cm }\end{array}$} & $\begin{array}{c}\text { Reduction versus } \\
\text { control, \% }\end{array}$ \\
\hline \multirow{4}{*}{$\begin{array}{l}\text { Richland, } \\
\text { Washington }\end{array}$} & Distilled water & 7.05 & $0.2 \pm 0.02$ & & 99,9999 \\
\cline { 2 - 4 } & Well water & 7.1 & $0.19 \pm 0.035$ & & 99,9999 \\
\cline { 2 - 4 } $\begin{array}{l}\text { Pasco, } \\
\text { Washington }\end{array}$ & Columbia River & 7.0 & $0.19 \pm 0.035$ & & 99,9999 \\
\hline \multirow{4}{*}{$\begin{array}{l}\text { St. Petersburg } \\
\text { Russia }\end{array}$} & Snake River & 7.0 & $0.19 \pm 0.03$ & \multirow{2}{*}{$2.5 \times 10^{6}$} & 99,9999 \\
\cline { 2 - 4 } & Spring Water & 7.1 & $0.18 \pm 0.025$ & & 99,9999 \\
\cline { 2 - 4 } & Distilled & 7.1 & $0.19 \pm 0.15$ & & 99,9999 \\
\cline { 2 - 3 } & Drinking & 7.0 & $0.2 \pm 0.03$ & & 99,9999 \\
\hline
\end{tabular}

PAEROSOL's impact on electronics (computers, monitors, cell phones, sensors, etc.), electrical appliances and fixtures (oscillating fans, heaters, lamps, etc.) and number of interior materials, such as title, colored polyethylene and polypropylene, patterned polyvinylchloride, patterned wallpaper, patterned cotton, synthetic and woolen fabric, lacquered and bare wood, aluminum, and stainless steel were studied during 4 consecutive months in a room of approximately $3,000 \mathrm{ft}^{3}$. PAEROSOL was used to regularly fumigate inside the room. After each fumigation the door remained closed (not sealed) for 16 to 20 hours. Upon completion of each PAEROSOL test, each item in the room was observed and the operation of electronics and electrical equipment was checked. All electronic and electrical equipment remained fully operational after 4 months and no visual deterioration was observed. Of all the materials exposed to PAEROSOL, we observed a slight discoloring only on the patterned cotton and woolen fabrics. All other materials had not changed. For this 4-month experiment, we chose a very conservative protocol, using much more PAEROSOL for each fumigation than it was required to decontaminate that room. We speculated that if no adverse impact was observed after such excessive PAEROSOL application, PAEROSOL could be considered as environmentally safe.

PAEROSOL toxicity was studied with outbreed male and female mice of different ages (external validity study) and with Swiss Webster specific pathogen-free female mice (internal validity study). Multiple controls were designed for each experimental group. Some experimental groups were directly exposed to a different concentration of PAEROSOL in an aerosol chamber (PAEROSOL was fumigated in their presence), and remained there while PAEROSOL desiccated. The corresponding control group was exposed to a microaerosol of table salt solution that has not been electrochemically activated. The other groups were introduced to the chamber at different stages of PAEROSOL desiccation. PAEROSOL desiccation was constantly monitored by on-line sampling aerosol from the chamber and analyzing aerosol particles size. Results of the PAEROSOL toxicity study conducted in the conditions simulating PAEROSOL application for disinfection of a confined environment revealed no toxic effects in mice directly and indirectly exposed to PAEROSOL. We observed that animal behavior and general conditions, the state of skin and hair, and the body and the internal organs' weight were not distorted after direct and indirect exposure to PAEROSOL. Macroscopic and microscopic examination of lung tissues revealed no pathology and all hematological parameters measured in mice remained within physiological range. No toxic effect of PAEROSOL to animals' immune system was recognized. Certain blood parameters indicated a stress effect, which is common for small animals under experimental conditions. 


\subsection{Objectives}

The goal of this study was to validate the previously observed bactericidal efficacy of PAEROSOL against common HAIs in a non-human subject trial within a hospital setting of Madigan Army Medical Center on Joint Base Lewis-McChord in Tacoma, Washington.

The objectives of the study were to:

- assess the effectiveness of the PAEROSOL application on reducing microbial viability on various inanimate hospital surfaces as compared to the natural decline of viable microbes on various inanimate hospital surfaces

- demonstrate that the PAEROSOL-caused reduction in microbial viability is not followed by a later increase in viability of microorganisms exposed to PAEROSOL (in other words, demonstrate extended incubation does not increase recovery of microbial species on the surfaces exposed to PAEROSOL)

- optimize PAEROSOL parameters for future deployment

- demonstrate PAEROSOL environmental safety, (i.e., impact to hospital interior materials and electronic equipment exposed during testing)

- evaluate the ease of PAEROSOL use (i.e., reliability of the equipment and logistics). 



\subsection{Experiment Description}

The PAEROSOL efficacy validation trial was performed inside the MAMC Department of Clinical Investigation Animal Surgical Suite (DCI). The trial was coupon-based and included four materials characteristically found in MAMC setting. The trial used approximately 350, 1-in. by 1-in.-size coupons of each material that had been individually inoculated with one of four microbial species, representative of the species found within MAMC, according to the review of the current MAMC antibiogram. The microbial load on each coupon was within the ranges recommended by MAMC.

Three independent variables were tested and observed in the trial:

- sodium chloride concentration for PAEROSOL production

- the volume of PAEROSOL dispersed inside the room

- the time necessary to complete disinfection.

To evaluate the interactions between these variables and PAEROSOL efficacy, we ran serial experiments altering these variables. We applied triple control scenarios:

- multi-day quantitative assessment of dried microorganisms' natural decline in viability on different materials in the test room

- quantitative assessment of microorganisms' natural decline in viability on control coupons that had not been exposed to PAEROSOL during each experiment

- quantitative assessment of microorganisms' decline in viability from exposure to the microaerosol of a sodium chloride solution that had not been electrochemically activated.

The decontaminating efficacy of PAEROSOL was determined by PAEROSOL dispersion inside the test room, where inoculated test coupons were pre-positioned. Outcome analyses assessed the effectiveness of the PAEROSOL application on reducing microbial viability on test coupons as compared to the natural decline in viability of microbes on identical test coupons.

Room temperature, humidity, and the concentration of free chlorine inside the test room were monitored, but not controlled during each experiment.

During the experiments, the furniture and electronic equipment remained inside the test room as they would under normal-use circumstances. The door to the test room was closed but not sealed, and the airhandling system was turned off.

\subsection{Test Room}

The test room (post-mortem room) was approximately $2,000 \mathrm{ft}^{3}$ (15 ft long by $13 \mathrm{ft}$ wide by $10 \mathrm{ft}$ high) and it contained the normal fixtures of an animal post-mortem room: a surgical table, washing facilities, shelves, cabinets, a table, chairs, surgical ceiling lamp, and posters (Figure 4.1a). The room also contained electronic equipment, such as a $\mathrm{pH}$ meter, a STEL electrochemical device, humidity/temperature sensors, a chlorine sensor, and a peristaltic pump (Figure 4.1). The room was located close to the laboratories and offices where the hospital employees continued their usual routine 
while PAEROSOL was tested. There was some laboratory equipment located in the hallway right near the test room door where moderate traffic and use of the equipment continued while PAEROSOL tests were in progress (Figure 4.1b).
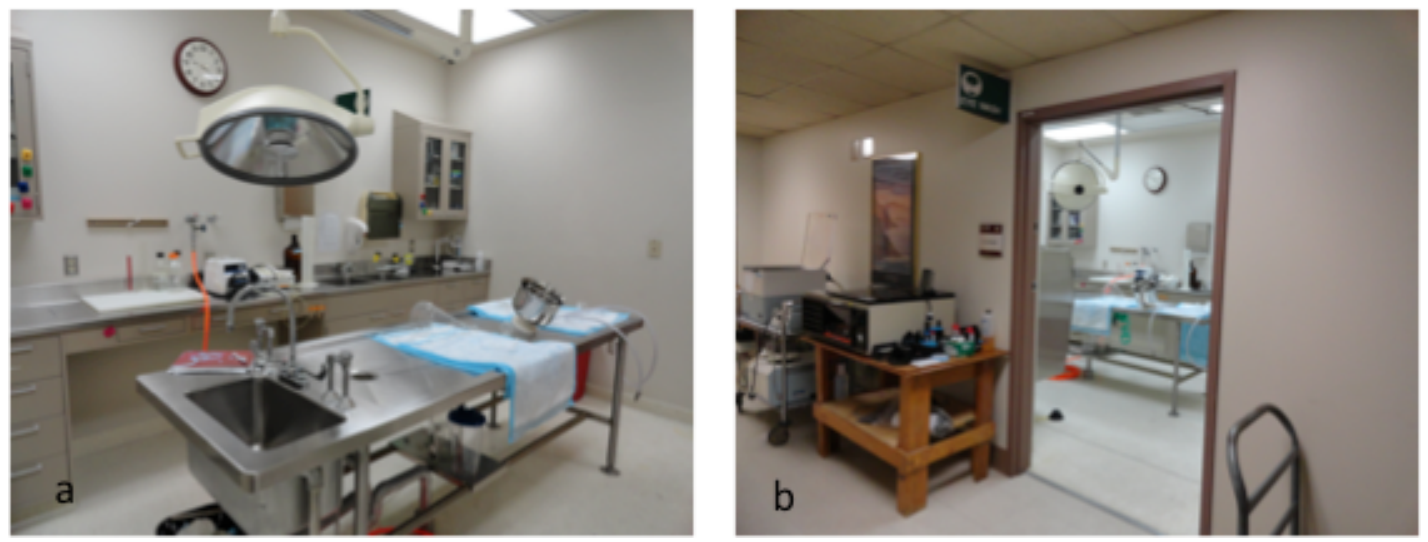

Figure 4.1. The Test Room at MAMC

For the control and PAEROSOL tests, three areas were designated and marked to realistically distribute coupons inside the room, including areas where PAEROSOL dispersion might be obstructed (e.g., under the table, under the shelves) (see Figure 4.2 and 4.3).

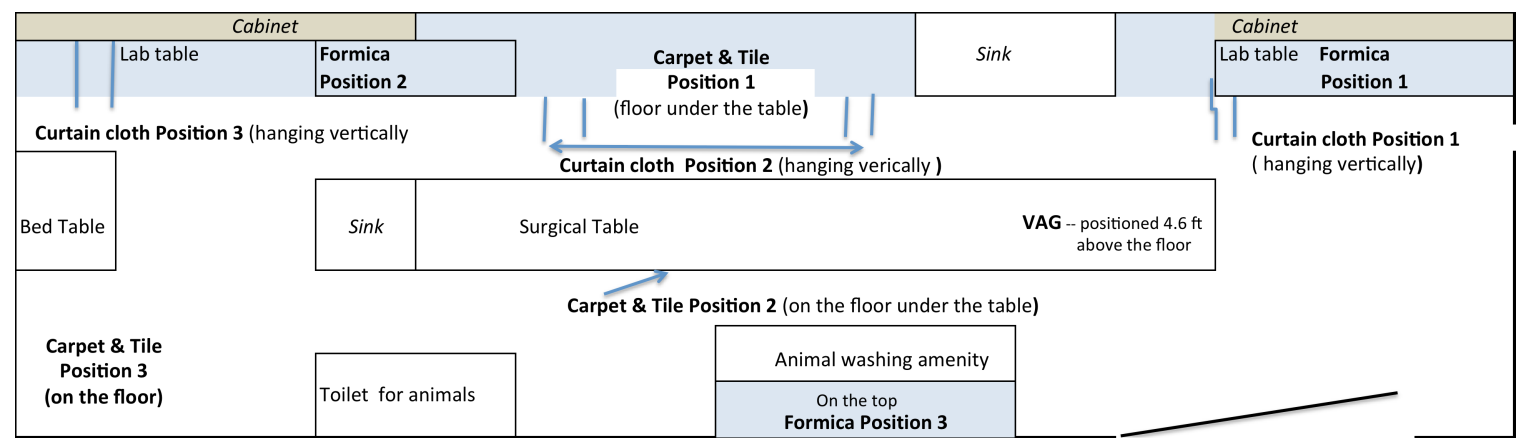

Figure 4.2. Schematic of the Test Room with Designated Areas for Coupon Placement
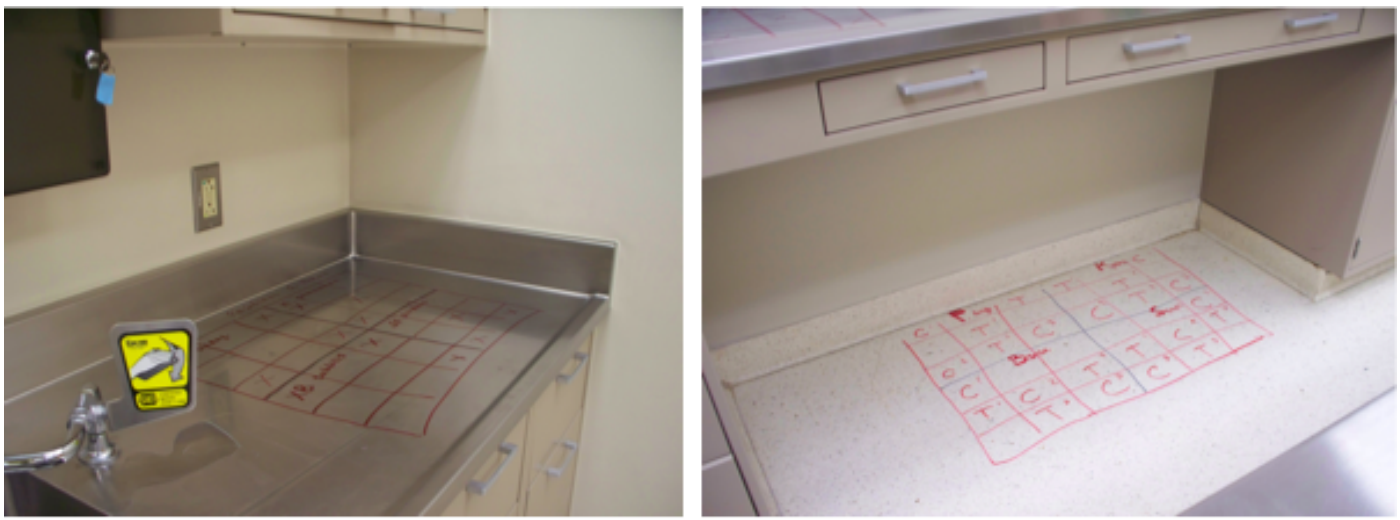

Figure 4.3. Examples of Designated Areas in the Test Room on Countertop and Under the Table 


\subsection{Tested Pathogens}

Three microbial species, representative of species found within MAMC, as per review of the MAMC 2009 antibiogram were provided by CAP Dr. Samandra Demons from the stock of the Laboratory of Microbiology of DCI, Madigan Healthcare System:

- Pseudomonas aeruginosa (Schroeter) Migula, isolated from blood culture (ATCC ${ }^{\circledR} 27853^{\mathrm{TM}}$ )

- Staphylococcus aureus subsp. aureus Rosenbach, clinical isolate (ATCC ${ }^{\circledR} 25923^{\mathrm{TM}}$ )

- Klebsiella pneumoniae subsp. pneumoniae (Schroeter) Trevisan (ATCC ${ }^{\circledR} 35657^{\mathrm{TM}}$ )

The fourth tested microorganism—Bacillus subtilis (Ehrenberg) Cohn, spores (ATCC ${ }^{\circledR} 49760^{\mathrm{TM}}$ )—was obtained from the PNNL collection to mimic Clostridium difficile spores, upon MAMC recommendation.

\subsection{Tested Materials}

MAMC provided four interior materials that are currently used in MAMC:

- Golden Mascarello Laminate Countertop Model \#: 3465-RD-30X096-000

- Armstrong Standard Excelon Imperial Texture Fortress White Vinyl Composition Commercial Tile

- Mohawk 100\% ColorStrand ${ }^{\circledR}$ Nylon. Model: 7879 Socratis

- AR Nelson fire-retardant 100\% polyester NFPA 701 Certified.

\subsection{Tested Equipment}

\subsubsection{STEL Electrolyzer Model 10N-120-01}

A STEL Electrolyzer Model 10N-120-01 (Bakhir Institute of Electrochemical System and Technologies 2012) with four FEM-3 electrochemical modules was used to produce the bactericidal anolyte. The operational stability and ease of use were examined through the trial.

\subsubsection{Proprietary Vortical Aerosol Generator}

The proprietary vortical aerosol generator (VAG) is a high-output atomizer generating 300-500 liters of PAEROSOL (particle diameter $0.5-10 \mathrm{~m} \mu$ ) per min. The VAG is stainless steel, lightweight (5-pound), portable ( $\varnothing 10$ in.; H 11 in.) device that has neither electronic, nor disposable parts. It operates through the standard hose by compressed air at 35-38 psi. The VAG operates at any temperature (where water is liquid) and humidity, and requires virtually no maintenance and no special skills to operate (Figure 4.5). Reproducibility of VAG operation was assessed as the volume of anolyte blended with air per minute. 


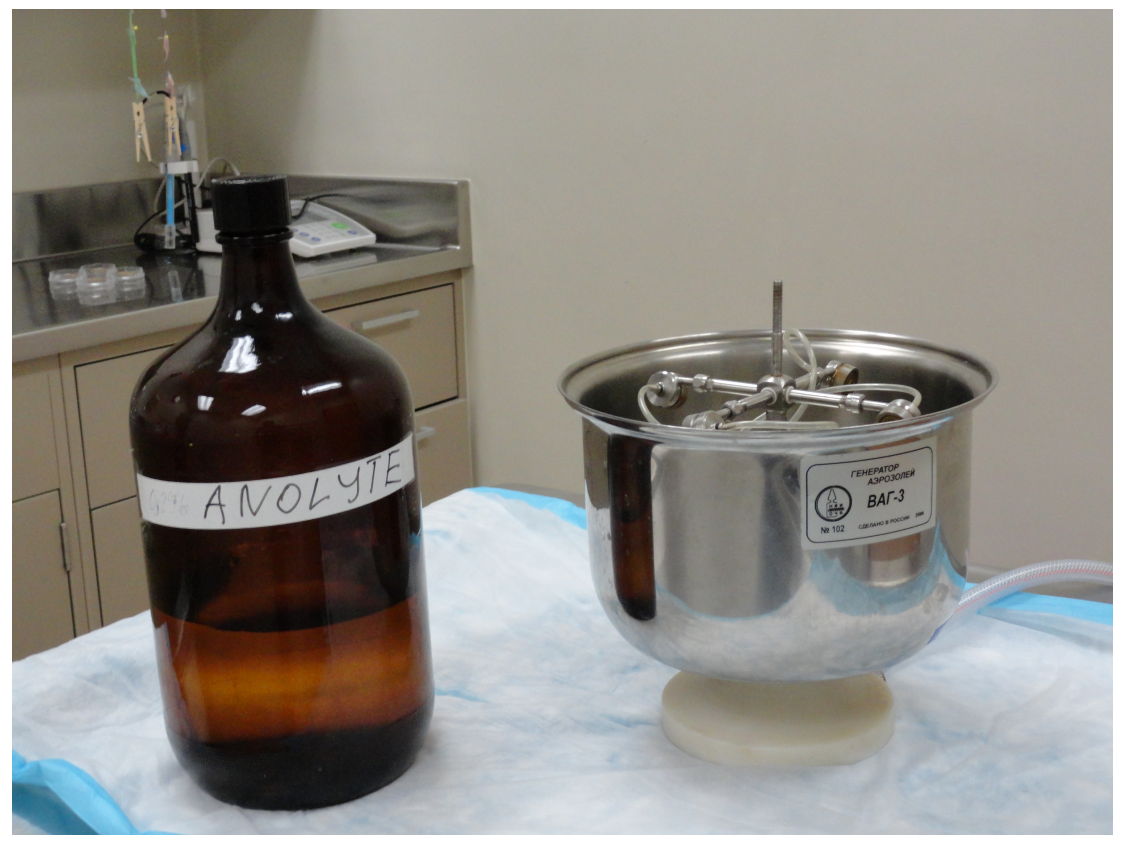

Figure 4.4. VAG Generator

\subsection{Trial Sequence}

The proposed objectives and the performance parameters dictated the sequence of the tests by which the PAEROSOL efficacy was evaluated. The PAEROSOL decontamination evaluation for this trial was limited to a quantitative assessment of its efficacy in reducing microbial viability on PAEROSOL-exposed test coupons positioned in real hospital conditions, as compared to the natural decline in viability on identical but non-PAEROSOL-exposed test coupons in the same hospital conditions.

First, we optimized the experimental protocol to achieve a reproducible recovery of each microorganism from each material. Next, we optimized the protocol to achieve the required level of contamination of each material by each microorganism to study the kinetics of their natural decline in viability in test room conditions. Finally, we optimized key parameters dictating PAEROSOL bactericidal efficacy, such as the $\mathrm{NaCl}$ concentration used for anolyte production, the volume of anolyte used for PAEROSOL production, and the time necessary to achieve a $3 \log _{10}-5 \log _{10}$ reduction in microbial load per coupon of each material positioned in the test room.

\subsubsection{Preparation of Test Coupons}

The 1-in by 1-in size of test coupons was chosen to simplify the recovery protocol and to increase recovery reproducibility by extracting microbes from the entire test coupon.

Each of the test coupons was cut to 1-in by 1-in from a large piece of test material. MAJ Michele A. Soltis, MC (the project principal investigator from MAMC) prepared approximately 150 coupons of each material. Additionally, approximately 200 coupons of each material were cut by RFP Plastics 
(of Kennewick, Washington) from large pieces of test materials supplied by Mr. Joseph Weston, Emergency Management Coordinator (MAMC).

Prior to use, all coupons were sterilized by wet heat. After wet heat sterilization, the coupons were allowed to dry completely in a biological safety cabinet (BSC). We observed no damage from the wet heat sterilization. Sterile coupons were kept in sterile containers. Before use, three randomly selected coupons of each material were extracted and plated to confirm sterility. Standard aseptic laboratory practice was exercised to prevent contamination of test surfaces during all phases of handling the test coupons. The test coupons were visually inspected prior to spiking (contaminating) with the microorganisms. Coupons with anomalies, such as chipped tile and Formica, frayed edges on carpet and curtain cloth were not used. Coupons were used once.

On each evaluation day, each coupon was placed into a sterile 47-mm plastic Petri dish and remained in the dish until extracted. The evaluation staff assigned a unique identifier code to each Petri dish, and the code was placed on the bottom and on the lid of the dish. The code indicated the material, the microorganism, and the zone in the test room where the coupon was placed. The same identifier code was placed on extraction vials and plates. Approximately 350, 1-in. by 1-in. size coupons of each material were used through the trial.

\subsubsection{Preparation of Microbial Cells and Spores}

Fresh cultures of $S$. aureus, $K$. pneumonia, and P. aeruginosa were grown on Becton, Dickinson and Company (B) trypticase soy agar with 5 percent sheep blood (TSA II), or on BD trypticase soy agar (soybean-casein digest agar) at $37^{\circ} \mathrm{C}$ for $18-24$ hours. Cultured plates were then extracted by washing cells from the plate with $5 \mathrm{ml}$ of $1 \mathrm{X}$ phosphate buffer solution (PBS). A 1:100 stock suspension of each culture was made by adding $50 \mu 1$ of stock suspension to $450 \mu 1$ of $1 \mathrm{X}$ PBS. The resultant suspension of each microorganism (approximately $10^{10} \mathrm{CFU} / \mathrm{ml}$ ) was then serially diluted, plated, incubated at $37^{\circ} \mathrm{C}$ for 18-24 hours, and enumerated to obtain the live microorganism concentration (CFU) in the stock suspension. The stock solution was either stored in a refrigerator at about $4^{\circ} \mathrm{C}$ for further use, or was further appropriately diluted with BD tryptic soy broth (TSB) for coupon inoculation. The CFU in TSB suspension was confirmed by serial dilution, plating, and enumerating after incubation at $37^{\circ} \mathrm{C}$ for 18-24 hours.

The suspension of B.subtilis spores in distilled water (approximately $10^{10} \mathrm{CFU} / \mathrm{ml}$ ) was heat-shocked at $80^{\circ} \mathrm{C}$ for 10 minutes, followed by an immediate cool down in crushed ice for 10 minutes, to kill residual vegetative cells. The spore suspension was serially diluted, plated, and incubated at $37^{\circ} \mathrm{C}$ for 18 hours to obtain the spore count in stock suspension. The spore suspension for coupon inoculation was prepared by the appropriate dilution of the stock solution with sterile distilled water. The stock spore suspension was stored at $4^{\circ} \mathrm{C}$ and was heat-shocked immediately before each spiking suspension was prepared.

\subsubsection{Spiking Test Coupons with Cells or Spores}

Each test coupon was placed in an individual Petri dish marked with an identification code and the Petri dishes were positioned flat in the BSC (Figure 4.5). Suspensions of microorganisms in TSB (which acted as an organic soil load) were used to inoculate coupons (Section 4.5.2). A 100- $\mu 1$ aliquot of a 
suspension (approximately $1 \times 10^{10} \mathrm{CFU} / \mathrm{ml}$ for the cells, and $1 \times 10^{6} \mathrm{CFU} / \mathrm{ml}$ for spores) was dispensed with a micropipette as $10 \mu 1$ droplets across the surface of the test coupon. After spiking with microorganism suspension, the test coupons remained undisturbed and uncovered until no moisture remained (1-2 hours) and then, dish lids were placed over the coupons. The dishes with coupons were carefully transferred to special plastic containers and stored undisturbed at room temperature before use. To confirm the CFU of each microorganism inoculated onto the coupons, the suspension of each microorganism used for coupon spiking was serially diluted, plated, and enumerated after incubation at $37^{\circ} \mathrm{C}$ for 18 to 24 hours.

\subsubsection{Recovery of Cells and Spores from the Test Coupons}

Microorganisms were extracted from the coupons with sterile PBS containing Tween 20 (PBST) (Valentine et al. 2008). Each coupon (not-exposed to PAEROSOL and exposed to PAEROSOL) was placed into sterile I-Chem* Field-Ready* Plastic Containers (Thermo-Scientific) pre-filled with $5 \mathrm{ml}$ PBST (Figure 4). Each container was vigorously mixed using a vortex mixer at high speed for 60 seconds to elute off viable bacteria or spores. The eluted suspension was serially diluted, plated onto TSA or TSA with $5 \%$ sheep blood, and enumerated after incubation at $37^{\circ} \mathrm{C}$ for $18-24$ hours. Longer than 24-hours incubation (48 hours) did not result in variations in CFU count as compared to 18-24 hours incubation. At least three plates for every dilution were cultured. For the test coupons exposed to PAEROSOL, undiluted extract was also plated by inoculating $100 \mu 1$ of undiluted extracts and by inoculating $4.5 \mathrm{ml}$ of undiluted extract. Following extraction of microorganism from the test, control, and blank coupons, extraction efficacy was further evaluated for each microorganism. Extracted coupons were washed with sterile water and then washed off water was inoculated to TSB and incubated at $37^{\circ} \mathrm{C}$ for 7 days. In parallel, washed off coupons were placed either onto TSA or TSA with 5 percent sheep blood (contaminated surface down) or were transferred into a liquid growth medium and incubated at $37^{\circ} \mathrm{C}$ for 7 days. Microorganisms' growth in liquid medium was visually observed daily and was analyzed spectrophotometrically at $550 \mathrm{~nm}$ on the day 7. The extraction containers were used once.
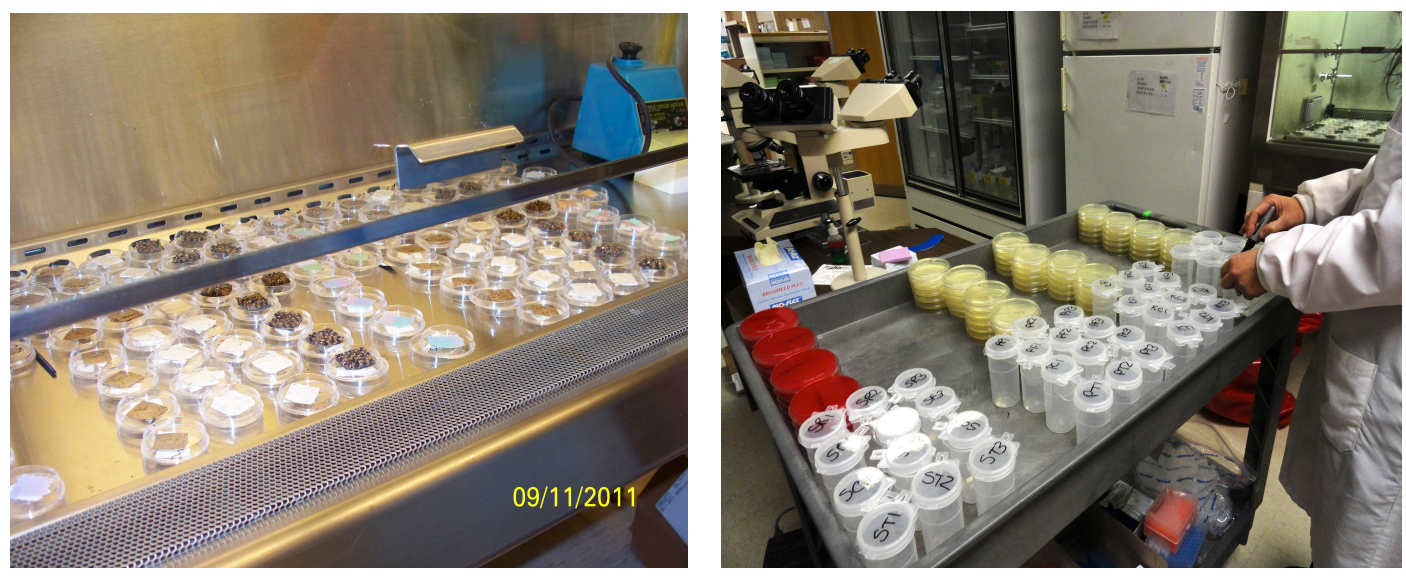

Figure 4.5. Coupon Preparation and Extraction

The efficacy of recovery of viable microorganisms from test, control, and blank coupons was ascertained in order to determine the differential number of microorganisms recovered from test coupons following initial spiking and remaining after completing the initial extraction process. The recovery of 
viable microorganisms was defined as the number of CFUs extracted from each coupon relative to the number of CFUs spiked onto each coupon. Extraction efficacy $(E x)$ was calculated for each of the triplicate coupons of each microorganism on each test material as: $E x=\log 10 \mathrm{~N} / \mathrm{N}^{\prime}$ where $N$ was the average number of viable organisms spiked on coupons and $N$ ' was the average number of viable organisms recovered from coupons.

\subsubsection{Preparation of a Liquid Disinfectant for PAEROSOL Test}

STEL Electrolyzer Model 10N-120-01 with four FEM-3 electrochemical modules was used to produce the bactericidal anolyte. This model, as well as all other STEL models, is designed to receive water directly from a water pipeline. For this standard regime, the internal STEL reservoir is filled with a high-concentration $\mathrm{NaCl}$ brine and an operator regulates the rate of brine supply to water flow to provide the required concentration of $\mathrm{NaCl}$ flowing to the FEM-3 modules for electrochemical activation. This regime is convenient when the same concentration of $\mathrm{NaCl}$ is required for a production of a large volume of electrochemically activated anolyte. However, for the production of small anolyte volumes, such as required for this trial (1.5-2 liters/per one PAEROSOL test), and for the production of the anolytes from the solutions of different $\mathrm{NaCl}$ concentrations, the STEL standard regime was inconvenient. Instead, in this trial the solution containing the required $\mathrm{NaCl}$ concentration $(0.125,0.25$, or 0.5 percent) was supplied to the STEL through a peristaltic pump (Figure 4.6).

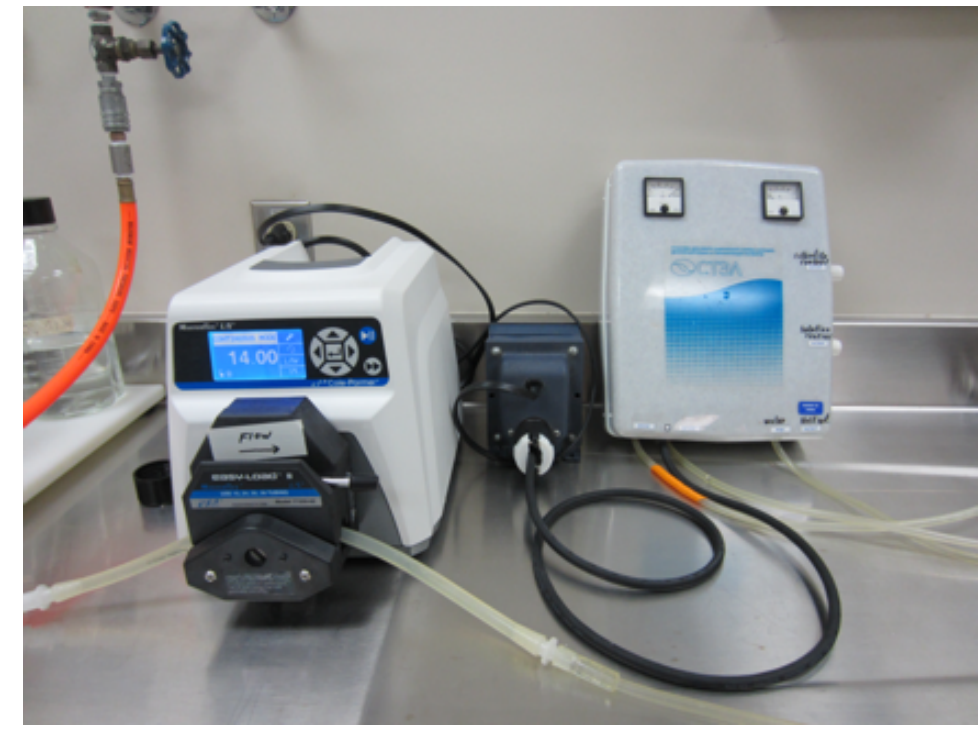

Figure 4.6. Photograph of STEL Device Connected to the Peristaltic Pump

The flow rate of $\mathrm{NaCl}$ solution through the STEL (FEM-3 modules), and the ratio anolyte/catholyte production - the factor that dictates anolyte $\mathrm{pH}$ - were adjusted once before the trial and remained unchanged through the testing in MAMC.

The concentration of $\mathrm{NaCl}$ was varied to produce anolyte with high ROS concentration from lowest $\mathrm{NaCl}$ concentration. At constant flow rate and the ratio anolyte/catholyte production, physical-chemical parameters of electrochemical activation directly depended on $\mathrm{NaCl}$ concentration in the solution introduced to the FEM-3 modules except oxidation-reduction potential (ORP) of the resultant anolyte was 
reduced by 4 percent and 7 percent when the $\mathrm{NaCl}$ concentration was decreased by a factor of two and four respectively. This fact is known for metastable electrochemically activated solutions, but not yet understood (Table 4.1). Disproportionate ORP reduction was also observed when freshly prepared anolyte was diluted by a factor of 10 and a factor of 100 (Table 4.2).

Table 4.1. Physical Chemical Parameters of the Anolytes Produced with Different Brines

\begin{tabular}{|c|c|c|c|c|c|c|c|c|}
\hline $\mathrm{NaCl}, \%$ & $\mathrm{U}, \mathrm{v}$ & $\mathrm{I}, \mathrm{a}$ & $\mathrm{Q}, \mathrm{l} / \mathrm{h}$ & $\begin{array}{l}\mathrm{V}_{\text {anol. }} / \\
\mathrm{V}_{\text {cathol. }}\end{array}$ & $\mathrm{pH}$ & ROS*, \% & ORP, mv & $\sigma, \mathrm{mS} / \mathrm{cm}$ \\
\hline 0.5 & 40 & $8.75 \pm 0.75$ & \multirow{3}{*}{14.0} & \multirow{3}{*}{$3 / 1$} & $6.97 \pm 0.13$ & $0.2 \pm 0.013$ & $942 \pm 13.4$ & $9.84 \pm 0.66$ \\
\hline 0.25 & $43 \pm 1$ & $5 \pm 0.05$ & & & $6.93 \pm 0.2$ & $0.11 \pm 0.1$ & $910 \pm 12.1$ & $5 \pm 0.43$ \\
\hline 0.125 & $46 \pm 1.5$ & $3 \pm 0.3$ & & & $6.95 \pm 0.1$ & $0.05 \pm 0.11$ & $880 \pm 12.4$ & $2.75 \pm 0.1$ \\
\hline
\end{tabular}

$\mathrm{U}=$ voltage imposed to FEM-3 modules

$\mathrm{I}=$ current in FEM-3

$\mathrm{ORP}=$ oxidation reduction potential of resultant anolyte

ROS $=$ measured concentration of reactive oxidative species in anolyte

$\mathrm{Q}=$ rate of brine flow through FEM-3 module

$\sigma=$ anolyte conductivity

Table 4.2. Physical-chemical Parameters of Diluted Anolytes

\begin{tabular}{|l|c|c|c|c|}
\hline \multicolumn{1}{|c|}{$\begin{array}{c}\text { Anolyte prepared from } \\
0.5 \% \mathrm{NaCl}\end{array}$} & ORP, $\mathrm{mV}$ & $\begin{array}{c}\text { ORP } \\
\text { Reduction, } \%\end{array}$ & ROS, \% & $\mathrm{pH}$ \\
\hline Undiluted (average of 5 different anolytes) & $950 \pm 13$ & & $0.2 \pm 0.015$ & $7.1 \pm 0.2$ \\
\hline Diluted by factor 10 & $916 \pm 14.3$ & 3.6 & $0.023 \pm 0.021$ & $7.1 \pm 0.2$ \\
\hline Diluted by factor 100 & $874 \pm 16.2$ & 8.0 & Below detection limit & $6.8 \pm 0.2$ \\
\hline
\end{tabular}

ORP $=$ oxidation reduction potential of resultant anolyte

ROS $=$ measured concentration of reactive oxidative species in anolyte

Disproportionate variation in ORP reduction and $\mathrm{pH}$ resulting from anolyte dilution was also observed by Cloete et al. (2009). One can see that the difference between ORP values in triplicate nondiluted and diluted anolytes was small (Table 4.2). A similar tendency was observed for the difference between ORP of the anolytes prepared from the solutions of higher and lower $\mathrm{NaCl}$ concentrations (Table 4.1). Contrary to ORP, ROS concentration in resultant anolytes was almost directly proportional to $\mathrm{NaCl}$ concentration and was reduced proportionally in diluted anolytes (Tables 4.1 and 4.2). Observed disproportions between ROS concentration and ORP in ECA anolytes remains unclear but raises at least two questions:

- Which of these two parameters better reflects the physical-chemical state of metastable electrochemically activated anolytes?

- Which of these two parameters determines its bactericidal efficacy (aka PAEROSOL efficacy)?

Our previous data (not published) and the results received through this trial allowed us to speculate that the ROS concentration in anolyte is the factor better reflecting anolyte/PAEROSOL germicidal efficacy. 
ORP, conductivity, and $\mathrm{pH}$ were analyzed with a $\mathrm{pH}$ meter (Seven Multi ${ }^{\mathrm{TM}}$ Mettler Toledo and corresponding probes). ROS concentration was analyzed by the thiosulfate-iodine titration method which is the redox titration often used to determine the concentration of mixed oxidizing agents (National Environmental Methods Index. Method\# 330.2). At least two aliquots of each anolyte were used, and the reproducibility of the results exceeded 99 percent. We also used perfectIONTM comb Cl Combination Electrode (Mettler Toledo) to analyze the ROS concentration in the anolyte. Electrode performance was not satisfactory possibly because of anolyte metastability. Unfortunately there is no reliable, accurate method allowing discrimination among different oxidants, specifically in the presence of chlorine.

A catholyte that was produced in parallel with anolyte in the proportion of 1:3, respectively, was collected, diluted with water, and drained. However, the catholyte may also find application in healthcare settings as an effective de-greaser and cleansing solution.

The germicidal efficacy of neutral anolyte $(\mathrm{pH}=$ approximately7) produced by the STEL electrolyzer was shown to be stable for at least 2-3 days at room temperature (or more than a week at $4^{\circ} \mathrm{C}$ ). However, in this trial fresh anolyte was prepared for each PAEROSOL test. This was done to avoid occasional "bad luck" with anolyte storage, and also, to evaluate STEL performance in frequent use. Potable and nonpotable water can be used for anolyte production (Table 2.2), but Milli-Q water was used in this trial to avoid biases that could be associated with MAMC water specifics.

\subsubsection{PAEROSOL Application}

For PAEROSOL tests, the coupons were delivered to the test room in special double containers. In the test room, covered Petri dishes were positioned according to the use of the coupon material: the floor tile and carpet coupons were placed horizontally on the floor; the countertop coupons were placed horizontally on the tables, and the curtain cloth coupons were pinned to ribbons (or clipped) and positioned vertically, as curtains would hang in a hospital cubicle (Figures 4.2 and 4.7).
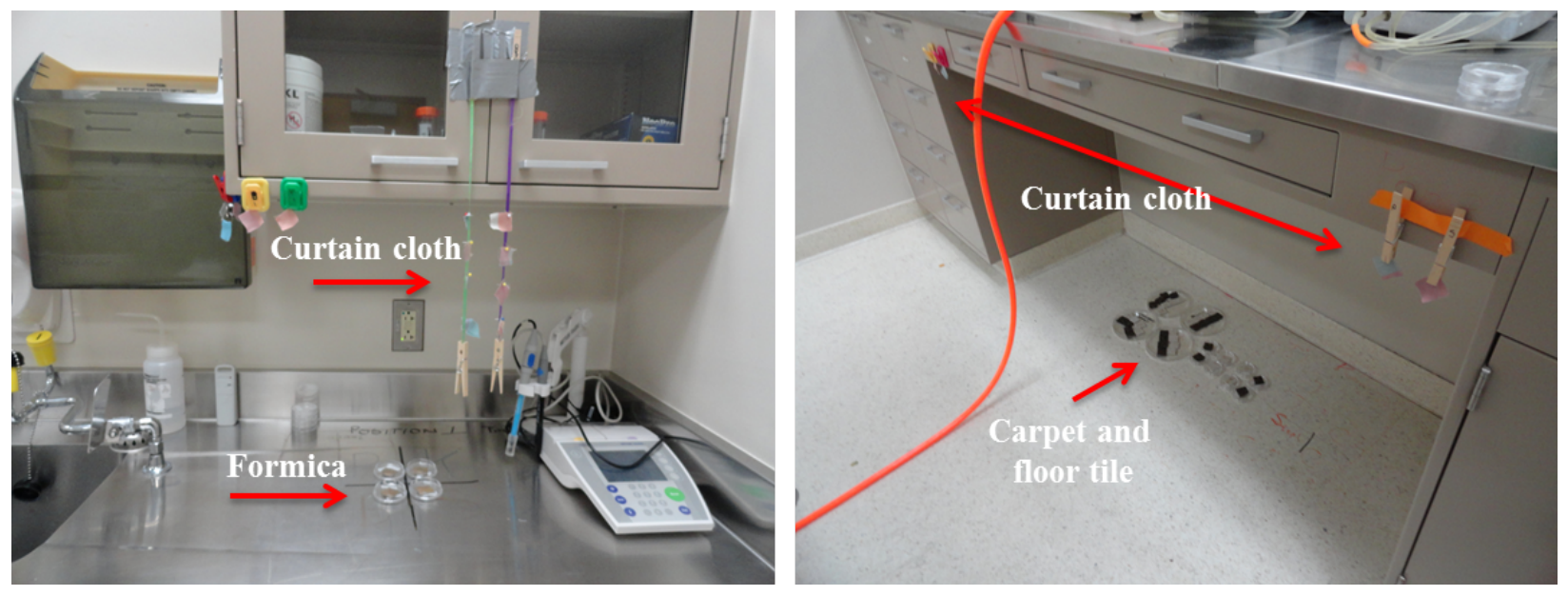

Figure 4.7. Arrangement of the Coupons in the Test Room 
The VAG PAEROSOL generator was prefilled with a pre-determined volume of freshly produced anolyte and connected to an in-room compressed air line through an ASCO solenoid, which allowed the remote control of the VAG through the closed door. Air pressure was controlled at 35 psi by a standard in-room regulator. The VAG was elevated $5 \mathrm{ft}$ above the floor to achieve quicker distribution of PAEROSOL across the test room. The lids were removed from the coupons, the door was closed (not sealed), and PAEROSOL generation was initiated remotely (Figure 4.8).

At $35 \mathrm{psi}$ and an airflow of 300-500 1/minute, the VAG atomized $60 \mathrm{ml} / \mathrm{minute}$ of anolyte and dispersed approximately $400 \mathrm{l} /$ minute of PAEROSOL containing $10^{10}-10^{12}$ reactive particles with a diameter in the range of $0.5 \mu \mathrm{m}$ to $10 \mu \mathrm{m}$. This VAG production capacity remained constant in each test, while the concentration of reactive PAEROSOL particles in the room was varied by dispersing different volumes of PAEROSOL. This parameter was optimized to achieve higher decontamination efficacy with a lower PAEROSOL volume dispersed inside the room.

Humidity and temperature inside the room were monitored, but not controlled, and the chlorine concentration was monitored in the hallway next to the room door.
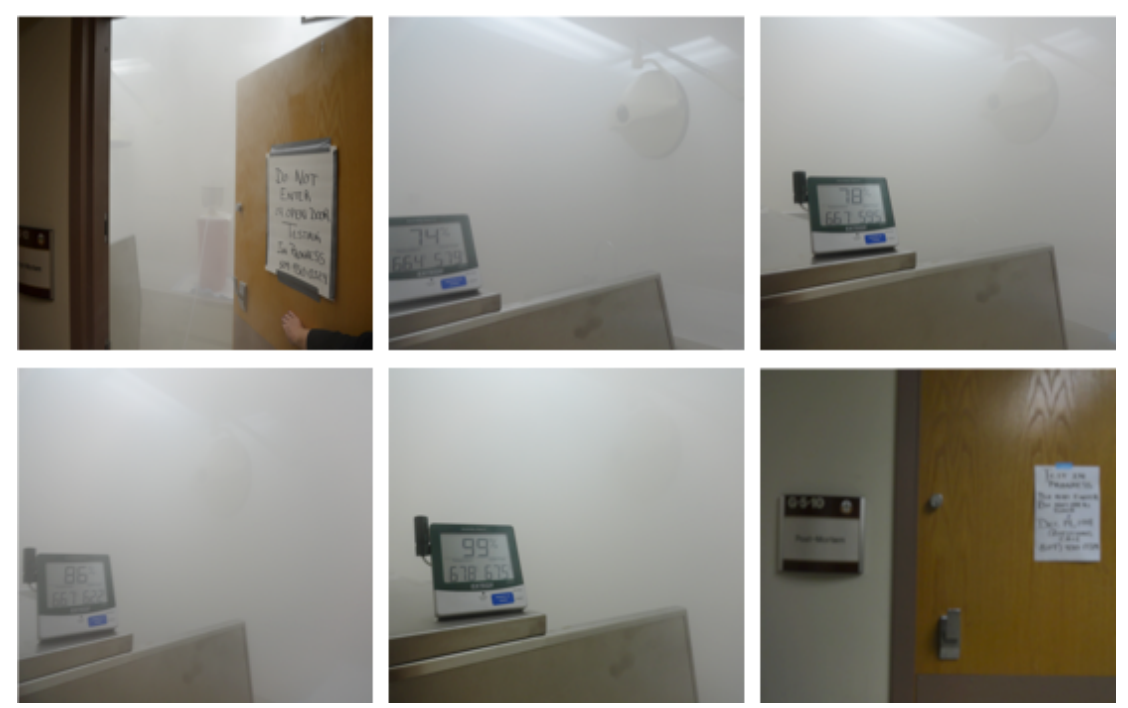

Figure 4.8. Illustration of PAEROSOL Dispersion Inside the Test Room

\subsubsection{PAEROSOL Efficacy Assessment}

For each microorganism inoculated on each material, the decontamination efficacy was calculated as the log reduction in viable organisms achieved by PAEROSOL treatment of the room. The efficacy (E), or $\log$ reduction, for all microorganisms was calculated as $\mathrm{E}=\log \left(N^{\circ} / N\right)$ where $N^{\circ}$ is the mean number of viable organisms applied to the control coupons (i.e., those not subjected to PAEROSOL treatment of the room), and $N$ is the number of viable organisms recovered from each test coupon after PAEROSOL treatment of the room. For samples where viable organisms were detected neither after plating $100 \mu 1$, nor $4.5 \mathrm{ml}$, the efficacy was calculated as the log of the mean number of viable organisms on the control coupons. Using the calculated $\log$ reduction for each test coupon, the mean log reduction (efficacy) $\pm \mathrm{SD}$ was calculated. 


\subsubsection{Assessment of $\mathrm{NaCl}$ Precipitation on Horizontal Surfaces Exposed to PAEROSOL}

To analyze $\mathrm{NaCl}$ precipitation on the surfaces after PAEROSOL application in the test room, open Petri dishes $(\varnothing 140 \mathrm{~mm})$ were positioned horizontally in different parts of the room, including nearby the coupons, before the test. Each dish had an identifier code. After the test completion, each dish was rinsed with $10 \mathrm{ml}$ Milli-Q water, and the rinsed liquid was transferred to individual vials. The conductivity in each vial was measured with an InLab®731 ISM-2m Conductivity Probe (Mettler Toledo) and $\mathrm{NaCl}$ concentration in rinsed solutions was calculated using a calibration plot.

The results of this assessment were also used to indirectly evaluate the PAEROSOL distribution through the room.

\subsubsection{Equipment Calibration}

All equipment (e.g., pipettes, incubators, and biological safety cabinets) used at the time of evaluation was verified as being certified, calibrated, or validated. The $\mathrm{pH}$ meters and corresponding electrodes were calibrated for each test according to the manufacturer's instruction. 



\subsection{Test Results}

PAEROSOL, a germicidal microaerosol, was evaluated for decontamination efficacy against three common hospital pathogens and one surrogate pathogen on four indoor surfaces in a hospital environment in DCI, MAMC. The evaluation followed a technology testing protocol approved by the MAMC Institutional Review Board (IRB) for the clinical investigation of a non-human subject trial.

PAEROSOL efficacy was assessed as the effectiveness of the PAEROSOL application on reducing microbial viability on test coupons compared to the natural decline in microbial viability on said test coupons.

\subsection{Efficacy of Microorganisms' Recovery from Contaminated Materials}

The efficacy of recovery of viable microorganisms from contaminated materials is a critical step in the evaluation of a decontamination technology. The number of microorganisms that can be recovered depends on the type of microorganism (i.e., the microorganisms' resistance to air drying), environmental conditions, and the specifics of material to which is the microorganism is applied, among other factors (Pettit and Lowbury 1968; Otter and French 2009).

The materials provided by MAMC for the PAEROSOL efficacy evaluation varied significantly. Non-porous floor tile with no pattern and lightly patterned non-porous countertop laminate were materials that were easy to cut, but their surface was hard to moisten uniformly with the microorganism suspension. Curtain and carpet coupons were easy to cut. However, both had loose fibers on the coupon edges. It was difficult to uniformly inoculate the carpet coupons because of the height of the loops (approximately $3 \mathrm{~mm}$ ), and the surface was quite hydrophobic. Of all the materials, the carpet was most difficult to inoculate. The curtain cloth was easy to inoculate because the microbial suspension immediately wet the cloth, possibly allowing spores to penetrate through the cloth to the Petri dish. We did not observe that liquid penetrated the cloth coupons to the Petri dish. Nevertheless, each Petri dish containing an inoculated cloth coupon was washed off with PBST and the resulting liquid was combined with extract from the coupon.

Consideration was given to achieve a satisfactory high load of each microorganism on each material for investigating the natural decline in viability in a hospital setting. HAI pathogens are known to be good survivors on inanimate surfaces, which might be attributed to mucus, saliva, or any other coating that accompany the pathogens naturally released from bodies. Biofoulers, such as $P$. aeruginos $a$ and K. pneumonia, easily survive in their biofilms. However, they desiccate and gradually die in environmental conditions when "artificially" inoculated on inanimate surfaces (Donlan and Costertan 2002; Anderl et al. 2000). Different strains of the same microorganism withstand desiccation differently in the environment (Pettit and Lowbury 1968).

Figure 5.1 and Table 5.1 demonstrate the cumulative results of the recovery of four microorganisms from the coupons of four materials. Based on previous practices and published data we expected B.subtilis spores to be more resistant to air drying than microbial cells. Therefore, spores were inoculated at a significantly lower concentration compared to vegetative cells. 
A significant reduction of viable microorganisms was observed for all cultures on all materials, except for $B$. subtilis spores. To discriminate between the efficacy of recovery and susceptibility to loss of viability because of desiccation caused by air drying (i.e., to understand whether or not viable microorganisms remained on the coupons after extraction), all extracted coupons were subjected to additional culturing that confirmed that no viable microorganisms remained on the coupons after extraction.

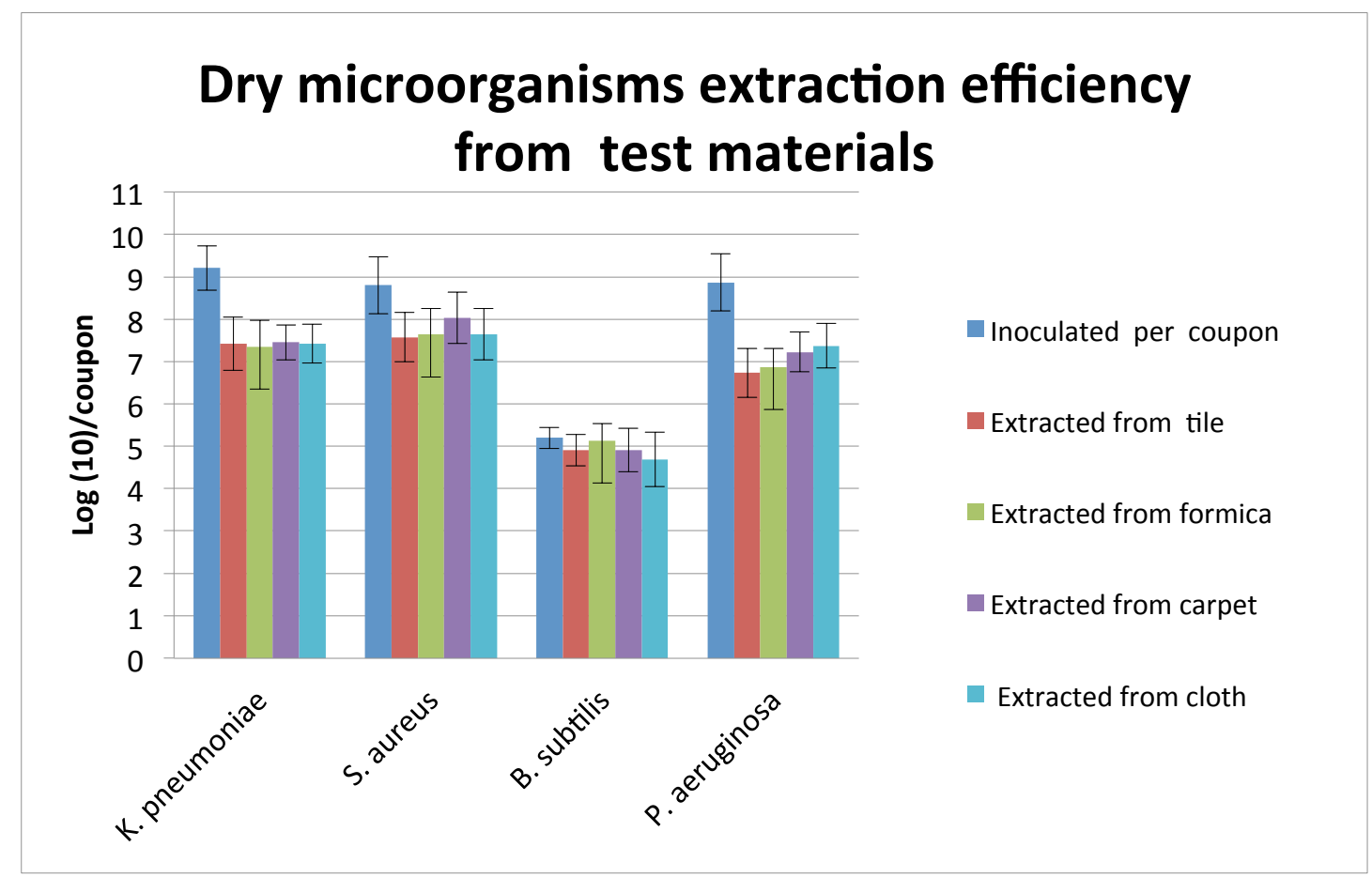

Figure 5.1. Recovery of Air-dried Microorganisms from Different Materials

From four tested cultures, $P$. aeruginosa appeared to be the most sensitive to desiccation, which is consistent with previously published data (Skaliy and Eagon 1972; Pettit and Lowbury 1968; Favero 2004). We observed no reliable variance in each microorganism's recovery from different materials and no significant difference in recovery of microorganisms (with the exception of B. subtilis) inoculated on the same material. Despite significant dissimilarity between test materials and non-controlled conditions during coupon preparation (temperature varied in the range between $21^{\circ} \mathrm{C}$ and $28^{\circ} \mathrm{C}$ and relative humidity $[\mathrm{RH}]$ ranged from 21 to 37 percent), the $\mathrm{CFU}$ of viable organisms that remained on the coupon was satisfactorily consistent and proportional to CFU inoculated on the coupons (Table 5.1). 
Table 5.1. Efficacy of Microorganisms’ Recovery from Contaminated Materials ${ }^{\mathrm{a}}$

\begin{tabular}{|c|c|c|c|}
\hline Test Material & Inoculum & Total Observed CFU & Mean $\log _{10}$ Reduction \\
\hline \multicolumn{4}{|c|}{ Klebsiella pneumoniae } \\
\hline Tile & $2.1 \times 10^{9} \pm 0.54$ & $4.2 \times 10^{7} \pm 0.37$ & 1.7 \\
\hline Formica & $2.1 \times 10^{9} \pm 0.54$ & $3.5 \times 10^{7} \pm 0.60$ & 1.8 \\
\hline Carpet & $2.1 \times 10^{9} \pm 0.54$ & $4.5 \times 10^{7} \pm 0.41$ & 1.7 \\
\hline Cloth & $2.1 \times 10^{9} \pm 0.54$ & $4.3 \times 10^{7} \pm 0.69$ & 1.7 \\
\hline \multicolumn{4}{|c|}{ Staphylococcus aureus } \\
\hline Tile & $8 \times 10^{8} \pm 0.37$ & $5.8 \times 10^{7} \pm 0.48$ & 1.1 \\
\hline Formica & $8 \times 10^{8} \pm 0.37$ & $6.3 \times 10^{7} \pm 0.43$ & 1.1 \\
\hline Carpet & $8 \times 10^{8} \pm 0.37$ & $3.0 \times 10^{7} \pm 0.61$ & 1.4 \\
\hline Cloth & $8 \times 10^{8} \pm 0.37$ & $6.6 \times 10^{7} \pm 0.61$ & 1.1 \\
\hline \multicolumn{4}{|c|}{ Pseudomonas aeruginosa } \\
\hline Tile & $8.7 \times 10^{8} \pm 0.34$ & $7.4 \times 10^{6} \pm 0.58$ & 2.1 \\
\hline Formica & $8.7 \times 10^{8} \pm 0.34$ & $8.7 \times 10^{6} \pm 0.44$ & 2.0 \\
\hline Carpet & $8.7 \times 10^{8} \pm 0.34$ & $2.2 \times 10^{7} \pm 0.47$ & 2.6 \\
\hline Cloth & $8.7 \times 10^{8} \pm 0.34$ & $3.7 \times 10^{7} \pm 0.5 .3$ & 2.4 \\
\hline \multicolumn{4}{|c|}{ Bacillus subtilis } \\
\hline Tile & $2.0 \times 10^{5} \pm 0.25$ & $9.0 \times 10^{4} \pm 0.37$ & 0.3 \\
\hline Formica & $2.0 \times 10^{5} \pm 0.25$ & $1.3 \times 10^{5} \pm 0.41$ & 1.2 \\
\hline Carpet & $2.0 \times 10^{5} \pm 0.25$ & $9.1 \times 10^{4} \pm 0.51$ & 0.3 \\
\hline Cloth & $2.0 \times 10^{5} \pm 0.25$ & $7.0 \times 10^{4} \pm 0.65$ & 0.5 \\
\hline
\end{tabular}

a. Data are expressed as mean ( \pm standard deviation) total number of microorganism (CFU) inoculated and observed, and $\log$ reduction due to air drying.

\subsection{Natural Decline in Viability in the Test Room Environment in the DCI at MAMC-positive Control Test (Coupons Contaminated but not Exposed to PAEROSOL)}

Microbial survival on inanimate surfaces depends not only on the type of microorganism, though it is an important factor, but also on the environmental conditions, such as RH and temperature (Neeley 2012). Therefore, preliminary results of microbial survival on test materials were investigated at PNNL, where contaminated coupons were placed in a BSC and observed during three consecutive days. We monitored the $\mathrm{RH}$ and temperature in the BSC daily. The RH ranged from 28 to 34 percent, and the temperature ranged from $67^{\circ} \mathrm{F}$ to $72^{\circ} \mathrm{F}$. Each day, two coupons of each material inoculated with each organism were extracted and processed (described in Section 4). The results obtained during this preliminary assessment were used to determine the appropriate number of viable organisms to be inoculated on each material to: 1) meet the MAMC recommendation on organism load per coupon $\left(10^{3}-10^{5} \mathrm{CFU}\right)$ and 2$)$ have enough viable organisms immediately after air drying to observe their natural decline in viability during extended exposure times to environmental conditions. 
To provide the foundation for the PAEROSOL efficacy assessment (positive control test), the observation of natural decline in viability of the four tested microorganisms on the four tested materials was conducted during three consecutive days in the MAMC test room. The test was conducted in compliance with the Test Protocol approved by MAMC IRB.

For the positive control test, 176 coupons (44 coupons for each culture) were simultaneously inoculated (as described earlier). The spiking suspension of each of four microorganisms were serially diluted, plated, and enumerated (described in Section 4) to accommodate the number of CFU to be inoculated on each coupon. Additionally, two coupons of each microorganism on each material were extracted one hour after no moisture remained on all coupons to account for CFUs remaining on the coupons after air drying (experiment time "0"). The extracted microorganisms were serially diluted, plated, and enumerated as described in Section 4.

One hundred and forty-four coupons, each in individual Petri dishes with assigned identifier codes were transferred to the test room in special containers and positioned in pre-determined areas as described for the PAEROSOL test. Three identical coupons of each microorganism inoculated on each material were positioned in each of the three areas designated for a particular material. The door was closed (not sealed). Every 24 hours, the door was opened, and one coupon from every coupon's locations was withdrawn (total of 36 coupons/day), covered by a corresponding lid, placed in the container, and transferred to the laboratory, where the coupons were extracted and plated. Each extracted suspension was serially diluted, and four dilutions were plated in triplicate. Overall, 432 samples were plated daily during the 3-day control trial. The results of the control trial are presented in Figures 5.2 through 5.5.

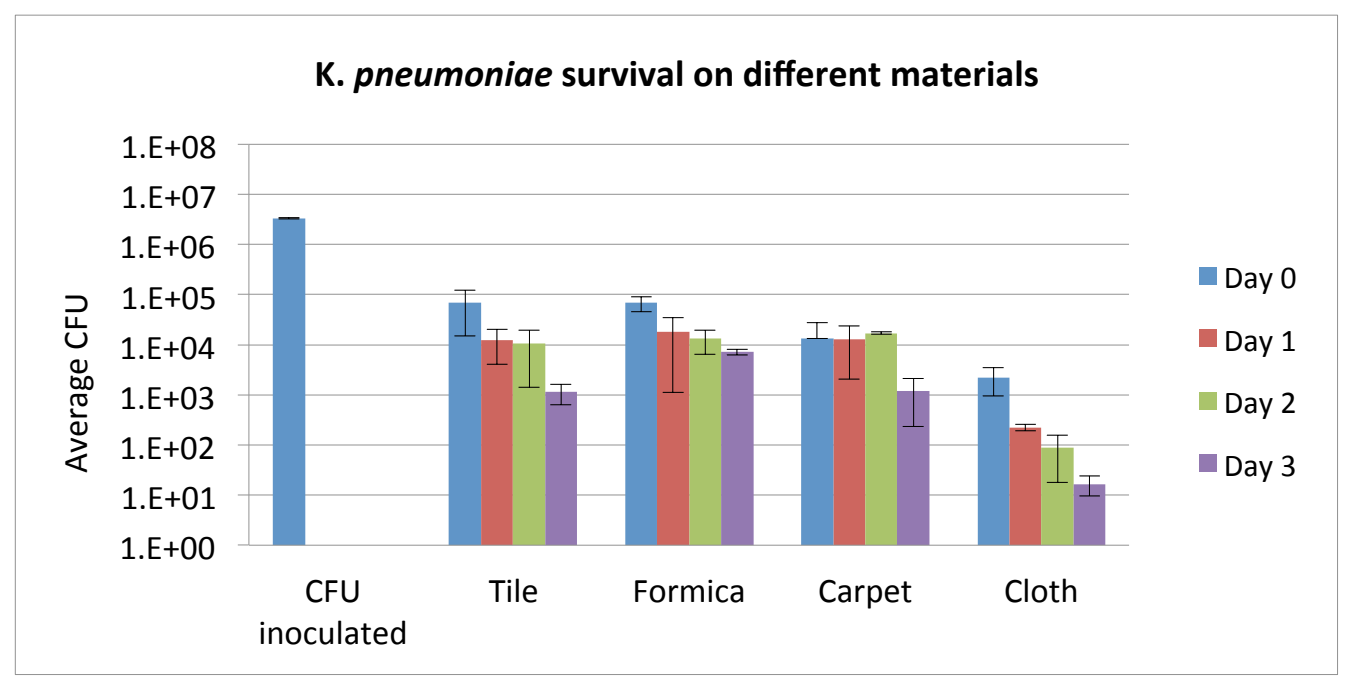

Figure 5.2. K. pneumoniae Survival on Different Materials in the Control Experiment 


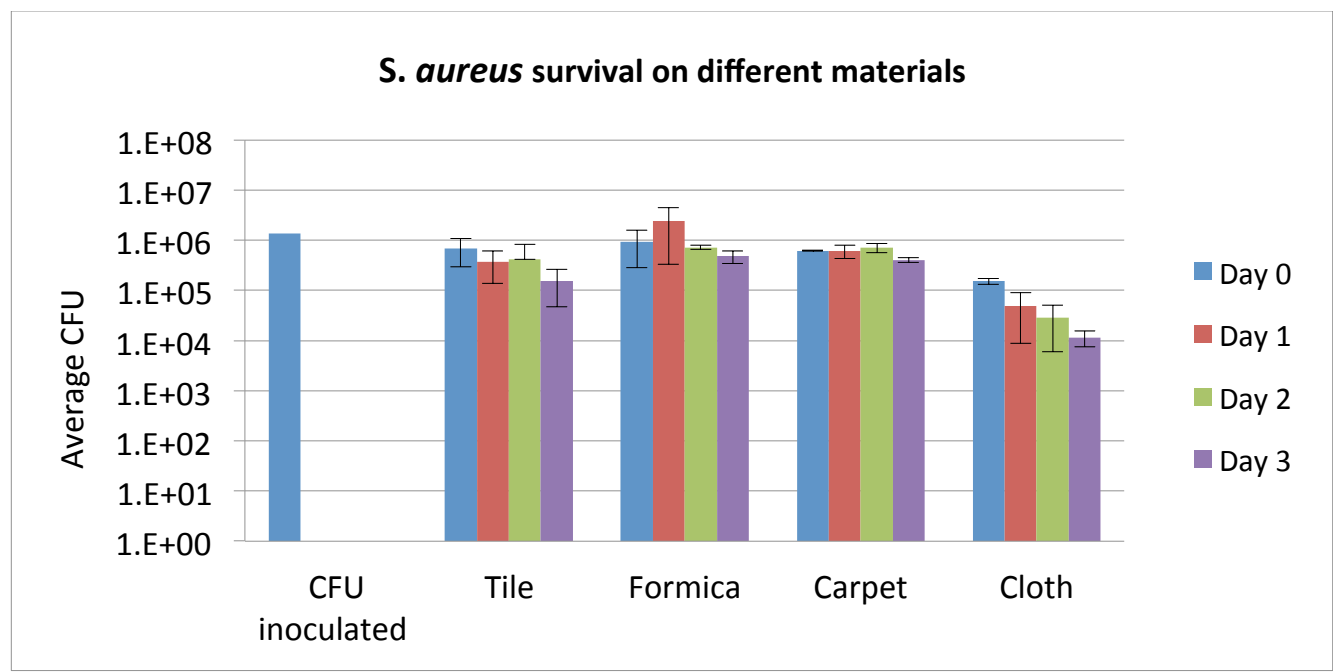

Figure 5.3. S. auerous Survival on Different Materials in the Control Experiment

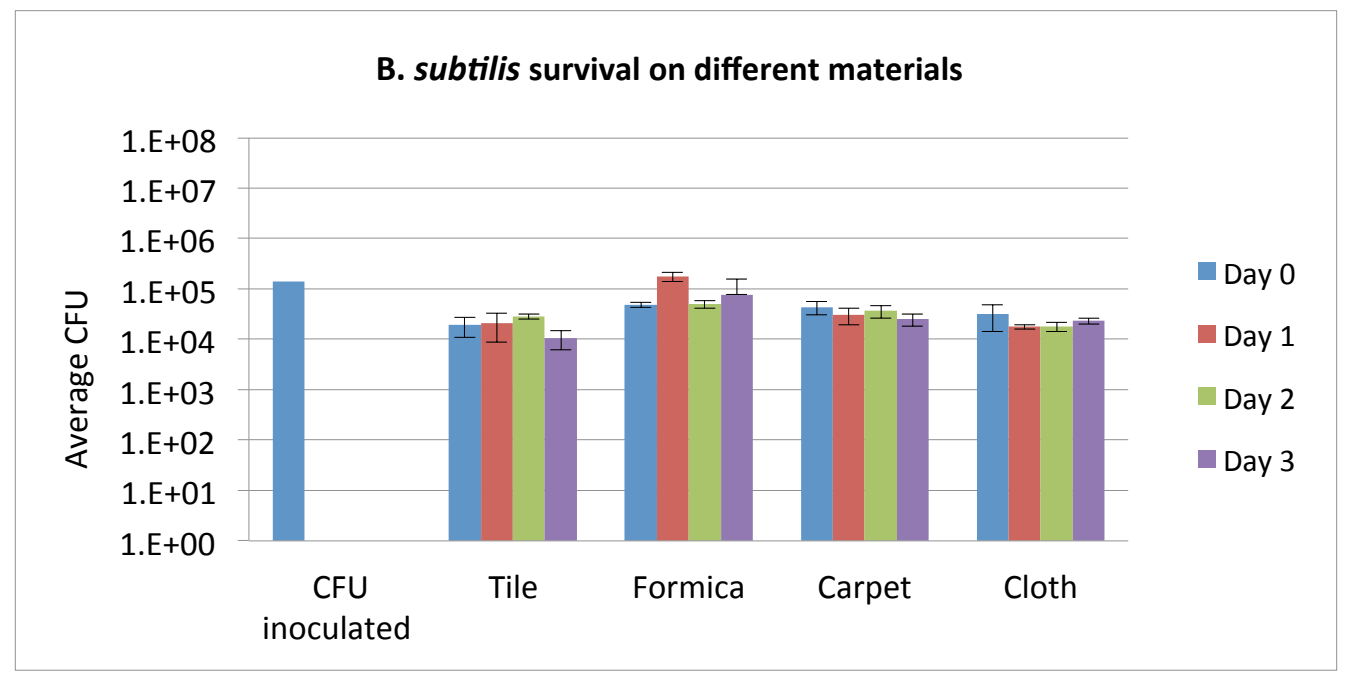

Figure 5.4. B. subtilis Survival on Different Materials in the Control Experiment 


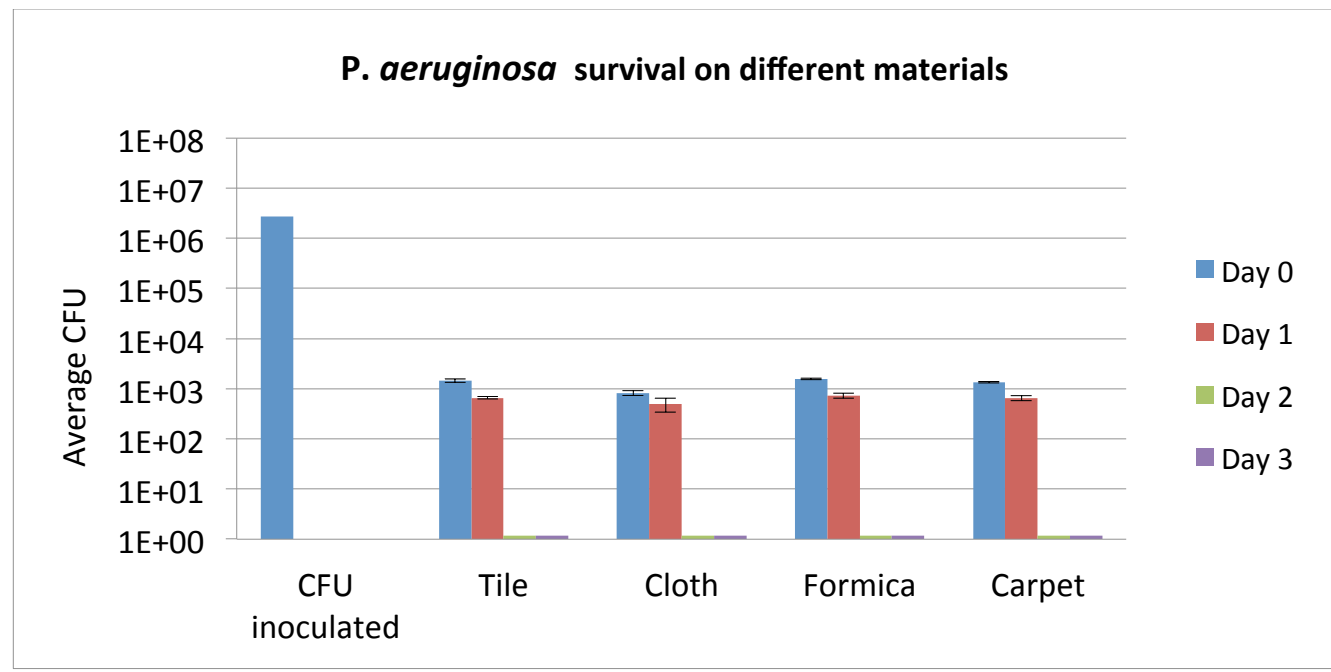

Figure 5.5. P aeruginosa Survival on Different Materials in the Control Experiment

No reliable variation was observed between coupons of the same microorganism inoculated on the same material but positioned in three different locations in the room. Therefore, the average of the number of microorganisms surviving on three identical coupons from three different positions was calculated and illustrated in Figure 5.2 through 5.5. Compared with the number of viable spores recovered from the test coupons of each material after air drying (Day 0), the number of viable B.subtilis spores declined insignificantly during three days on all tested materials. Persistence of $S$. aureus on all tested materials was quite high, except on curtain cloth, where the number of viable cells reduced steadily for $1.0 \times \log _{10}$ during three consecutive days. Significantly lower resistance to environmental conditions was observed for $K$. pneumoniae. During three days, the reduction in the number of viable K. pneumoniae equaled 95 percent on the curtain cloth, 98.6 percent on tile; and 90 percent on Formica. On carpet coupons, the reduction in viability was about 90 percent during two days and dropped to about 1 percent on the third day. The highest susceptibility to environmental conditions was observed for $P$. aeruginosa. Viable cells were recovered from the coupons only after 24 hours. On the second and third day after the test began, 1-2 colonies were observed when $100 \mu 1$ of non-diluted suspension extracted from the coupons was inoculated. P. aeruginosa's high susceptibility to desiccation was reported by Skaliy and Eagon (1972). RH and temperature in the test room were monitored once a day when the coupons were withdrawn from the room. RH variations were in the range 28 to 32 percent, and temperature ranged from $67.5^{\circ} \mathrm{F}$ to $71^{\circ} \mathrm{F}$.

\subsection{Evaluation and Optimization of PAEROSOL Application}

Optimization of PAEROSOL application aimed to reduce the concentration of $\mathrm{NaCl}$, the volume of PAEROSOL (i.e., volume of anolyte), and time required to complete the decontamination process. It was previously recognized that the combination of four factors dictates germicidal efficacy of PAEROSOL:

1. the size of PAEROSOL particles

2. concentration of ROS in the anolyte

3. volume of PAEROSOL disseminated inside a confined environment 
4. the time of contact between contaminated surfaces and PAEROSOL after PAEROSOL dispersion.

Empirical variation of these parameters brought some understanding, but not complete, of how these four factors interact with each other. For example, it was not possible to decrease ROS concentration in the anolyte by a factor of two and compensate decontaminating efficacy by dispersion of a symmetrically higher volume of PAEROSOL because of the humidity that grew proportionally to PAEROSOL volume and compromised the size of PAEROSOL droplets (e.g., caused droplets to agglomerate). It also was impossible to reduce the time required for the contact between contaminated surfaces and PAEROSOL by the distribution of higher volumes of PAEROSOL.

One factor - the size of the particles in the range of $0.5-10 \mu \mathrm{m}$ - was identified previously as providing both favorable surface-to-volume ratio (e.g., high ROS offgassing) and uniformity of PAEROSOL distribution inside a confined environment. However, humidity developed by PAEROSOL dispersion inside a confined environment also played an important role in PAEROSOL efficacy. At low humidity, PAEROSOL droplets desiccate too fast. At high humidity, which reflects a high volume of dispersed PAEROSOL, particles collide and drop down on horizontal surfaces, reducing the concentration of the particles in the gas phase. Therefore, empirical alteration of these factors in realistic environmental conditions in the test room was especially challenging.

Basically, only one factor was not changed through the trial: the size of the PAEROSOL particles produced by the VAG. In each run, it was controlled by a constant rate of anolyte conversion to PAEROSOL as $60 \mathrm{ml} / \mathrm{min}$. at the airflow rate of $400 \mathrm{~L} / \mathrm{min}$. at 35-37 psi. Also, the in-room air-handling system was shut down during each run, and the air outlet was covered with cardboard taped on the perimeter. This was done to build up PAEROSOL concentration within the room and prevent transport of PAEROSOL to other hospital facilities via the heating, ventilation, and air-conditioning (HVAC) system. Because a non-human subject trial was approved by MAMC, it was critical to prevent PAEROSOL transport to the other MAMC settings with occupants. The test room door remained closed but not sealed.

Three independent variables were observed in the trial: 1) $\mathrm{NaCl}$ concentration for PAEROSOL production, 2) the volume of PAEROSOL dispersed inside the room, and 3) the time necessary to complete disinfection. To optimize the parameters for PAEROSOL application, serial experiments were run altering these variables. Room temperature, humidity, and concentration of oxidative species in the hallway (measured as $\mathrm{Cl}$ concentration in the air) were monitored but not controlled during each experiment.

\subsubsection{Test Serial 1}

The first test was planned to observe the decontaminating performance of a low PAEROSOL volume during a very long contact time with contaminated materials. Figure 5.3 illustrates decontamination efficacy of a low volume of PAEROSOL $\left(0.35 \mathrm{ml}\right.$ of anolyte per $\left.\mathrm{ft}^{3}\right)$ during 24 hours of contact with contaminated materials.

During 11.6 minutes, $700 \mathrm{ml}$ of the anolyte was dispersed in the test room $\left(0.35 \mathrm{ml}\right.$ of anolyte per $\left.\mathrm{ft}^{3}\right)$, and contaminated coupons remained undisturbed in the test room environment for 24 hours. 


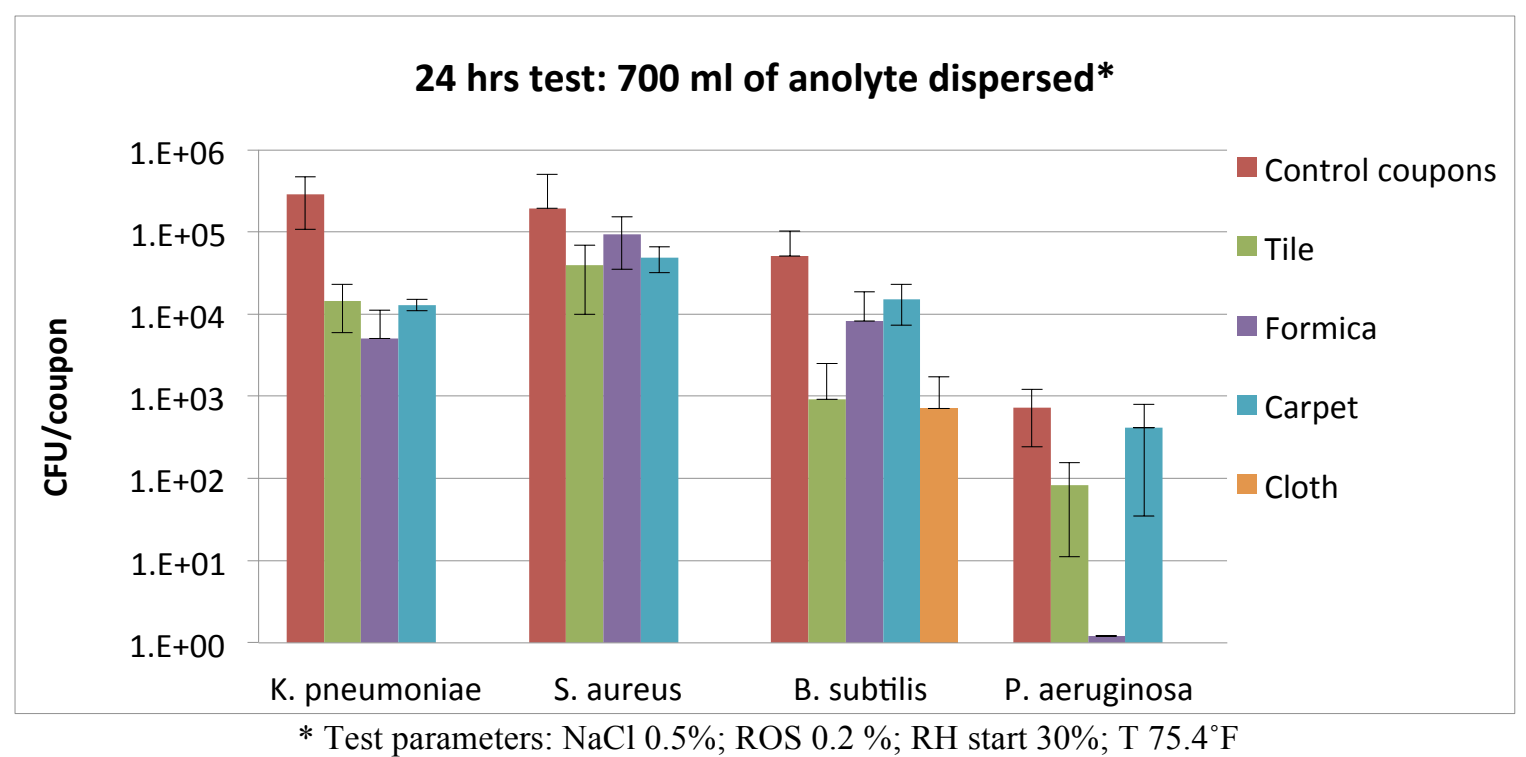

Figure 5.6. PAEROSOL Performance During 24 hours after Fumigation of $700 \mathrm{ml}$ of Anolyte

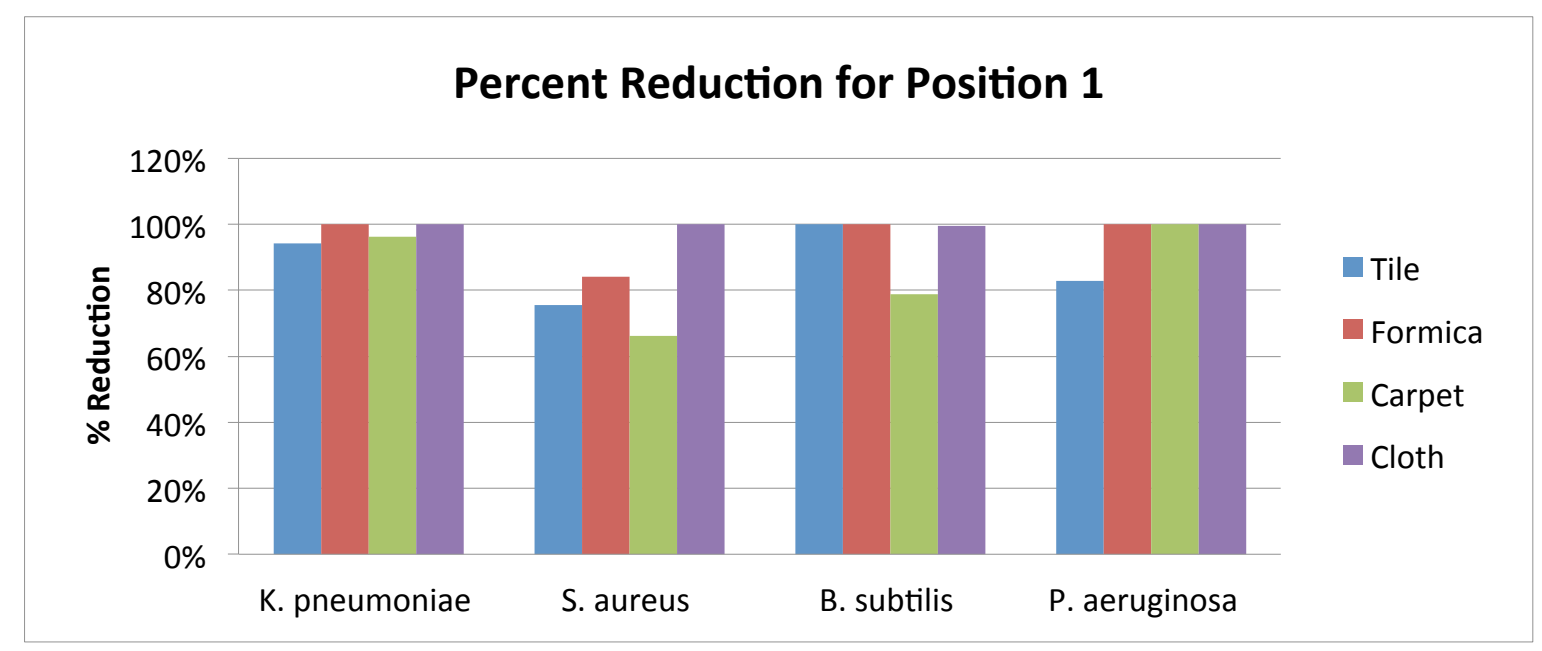

Figure 5.7. Percent Reduction for each Microorganism on each Material in Position 1 
B2

\section{Percent Reduction for Position 2}

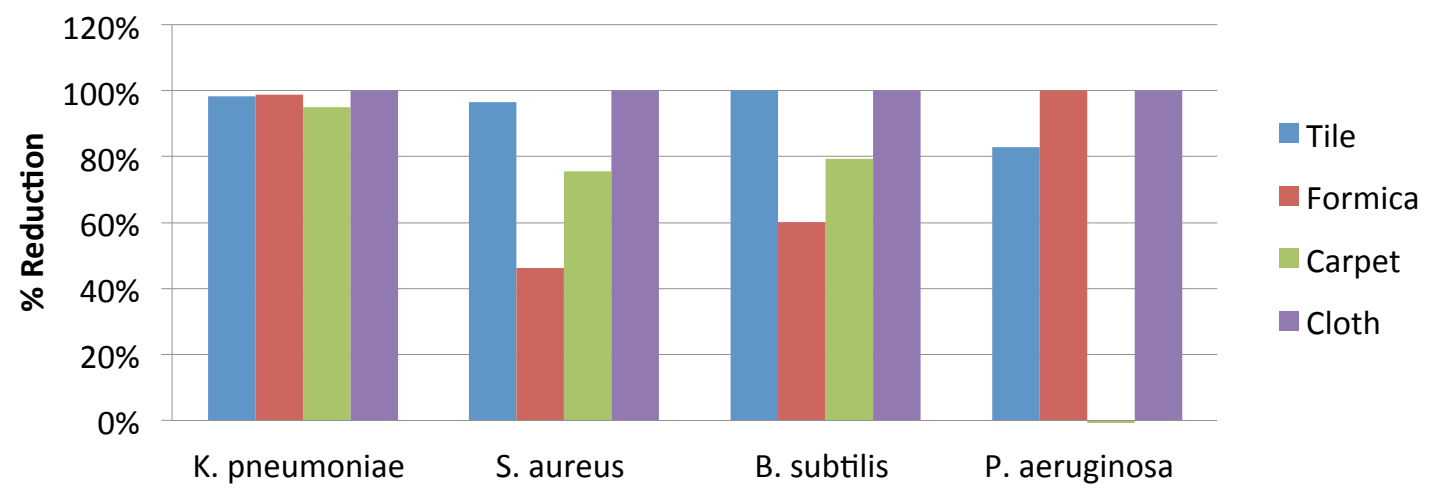

Figure 5.8. Percent Reduction for each Microorganism on each Material in Position 2

B3

\section{Percent Reduction for Position 3}

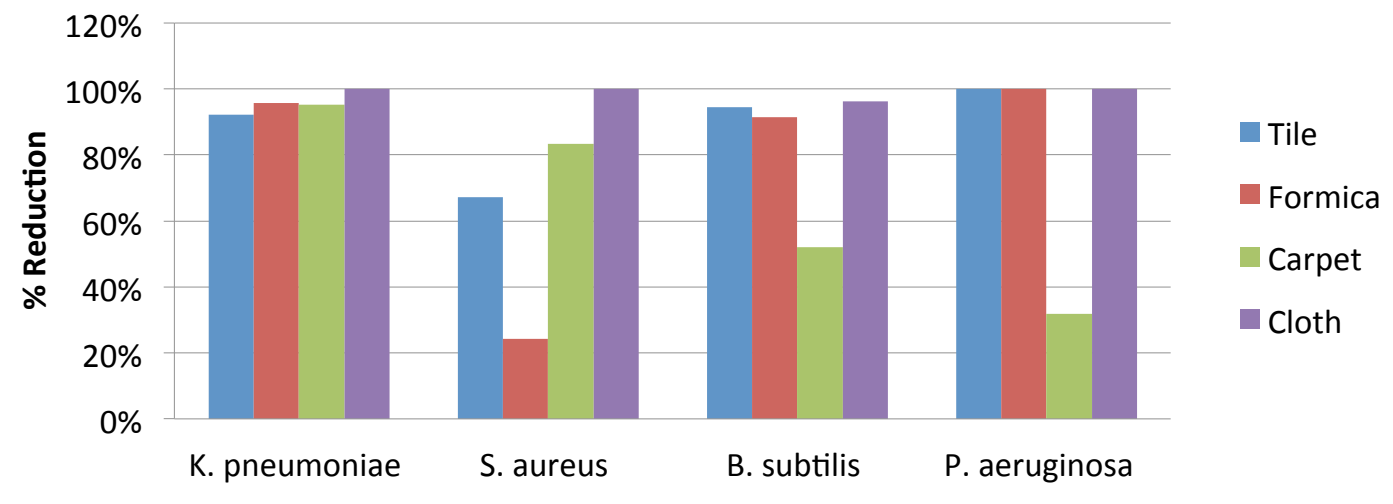

Figure 5.9. Percent Reduction for each Microorganism on each Material in Position 3 


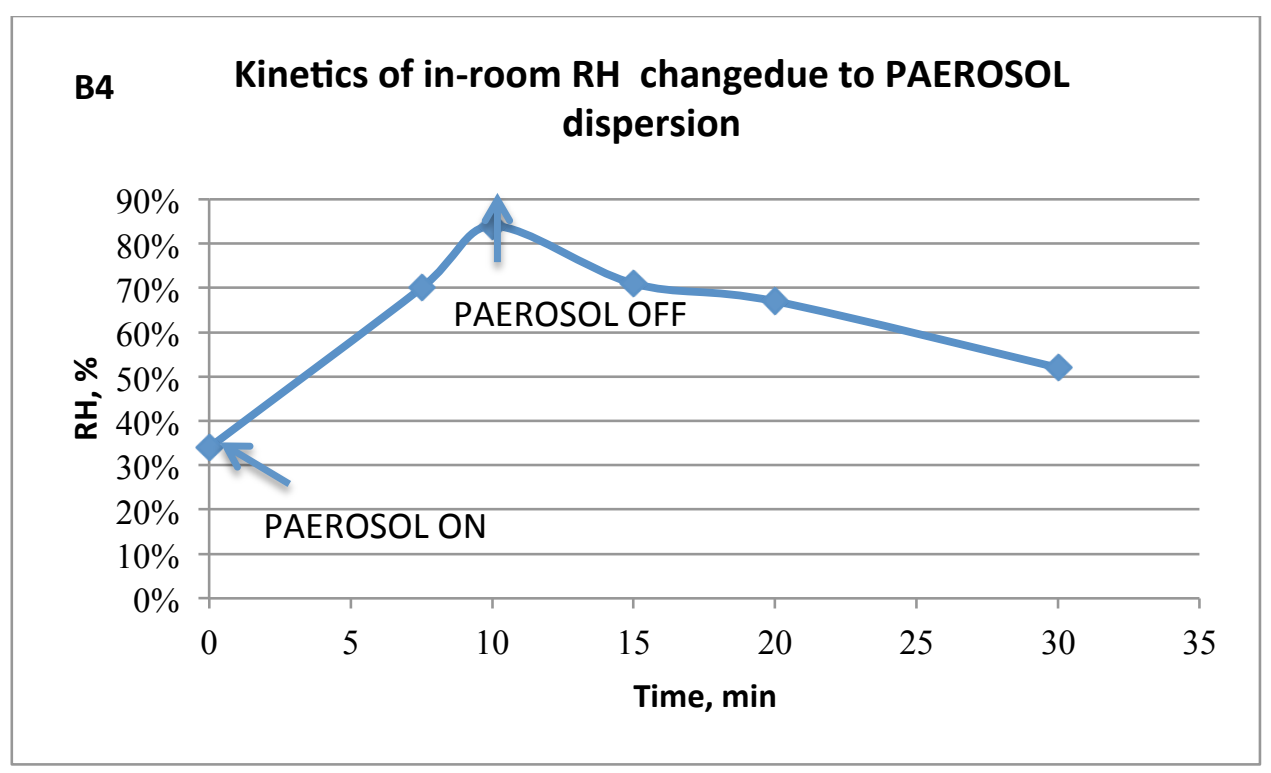

Figure 5.10. Kinetics of In-room RH Change after PAEROSOL Dispersion

Figure 5.6 represents the average of viable CFU of each microorganism that remained on identical coupons positioned in three different locations inside the test room. Figures 5.7 through 5.9 demonstrate the percent of reduction of each microorganism on each material positioned in each of three predetermined locations for each material. It should be noted that the number of the position does not mean that all coupons assigned to a particular number were located in the same place in the room. As indicated in Figure 4.2, carpet and floor tile coupons had the same positions 1, 2, and 3. Formica and curtain cloth coupons were distributed among three different positions from the ones for carpet and tile coupons (as described in Section 4).

As shown in Figure 5.6, no viable microorganisms were detected on curtain cloth coupons, except for B. subtilis, while the lowest reduction in CFU was achieved on carpet. On Formica, the average of reduction for all microorganisms except $P$. aeruginosa was in the range $1.0 \times \log _{10}-1.5 \times \log _{10}$. On tile coupons, even the supersensitive-to-desiccation $P$. aeruginosa survived. Significant differences in the CFU of the organisms reduced on the coupons of the same materials positioned in different locations was observed (Figures 5.7 through 5.9). It can be speculated that the volume of dispersed PAEROSOL was too low to provide for uniform PAEROSOL distribution (particles balance) inside the test room before the particles desiccated. Figure 5.10 shows the rapid increase of RH in the room because of PAEROSOL distribution, followed by quick RH reduction after PAEROSOL dispersion was completed. This fact indicated leakage of PAEROSOL to the hallway through the gap below the door. The air outlet inside the room was closed while PAEROSOL was produced and distributed inside the room at the rate of $400 \mathrm{l} / \mathrm{min}$. Therefore, the PAEROSOL leak through the gap under the door to the hallway was predictable. However, a PortaSens 22 Gas Detector Model C16 (Analytical Technology, Inc.) did not detect any $\mathrm{Cl}$ in the hallway.

\subsubsection{Test Serial 2}

Based on the results of the 24-hour test, two parameters for interrogation of PAEROSOL efficacy were varied. The time of PAEROSOL decontamination was reduced to 4 hours, while the volume of 
PAEROSOL was increased to $1340 \mathrm{ml}\left(0.67 \mathrm{ml} / \mathrm{ft}^{3}\right)$. The concentration of $\mathrm{NaCl}$ for anolyte production remained unchanged ( 0.5 percent). In Test Serial 2, $1340 \mathrm{ml}$ of anolyte were atomized to PAEROSOL during 22.3 minutes followed by 4 hours during which coupons remained undisturbed inside the room.

Although viability of every microorganism on every material was reduced, the overall reduction of the microorganisms in these conditions remained poor. A significant number of viable microorganisms remained on all materials positioned horizontally. On the cloth coupons that were hanging vertically, three out of four microorganisms were killed below detection level. Only viable B. subtilis spores remained on the cloth. The room's RH increased up to 99 percent because of PAEROSOL dispersion, and then it gradually dropped down to 35.2 percent over 4 hours. Despite PAEROSOL leaking from the test room to the hallway during PAEROSOL production, $\mathrm{Cl}$ concentration was not detectable in the hallway. The low performance of PAEROSOL was explained by the excessive volume of PAEROSOL distributed during the short time, which increased the incidents of particles colliding, followed by precipitation of large particles on horizontal surfaces. This resulted in a reduction of the number of active small particles in the air phase. Also, precipitated liquid prevented direct contact between microorganisms and ROS offgassing PAEROSOL particles. Previously, we observed that PAEROSOL efficacy was significantly lower when a spiking suspension of $E$. coli on glass coupons was not completely dried off before the contaminated coupons were exposed to PAEROSOL.

The volumes of anolyte and protocol for PAEROSOL dispersion were modified for later tests because $700 \mathrm{ml}$ of dispersed anolyte $\left(0.35 \mathrm{ml} / \mathrm{ft}^{3}\right)$ resulted in poor decontamination (Figure 5.6) and an almost twice as high volume of dispersed anolyte, $1340 \mathrm{ml}\left(0.67 \mathrm{ml} / \mathrm{ft}^{3}\right)$ also showed poor performance (Figure 5.11), although it was because of opposite reasons.

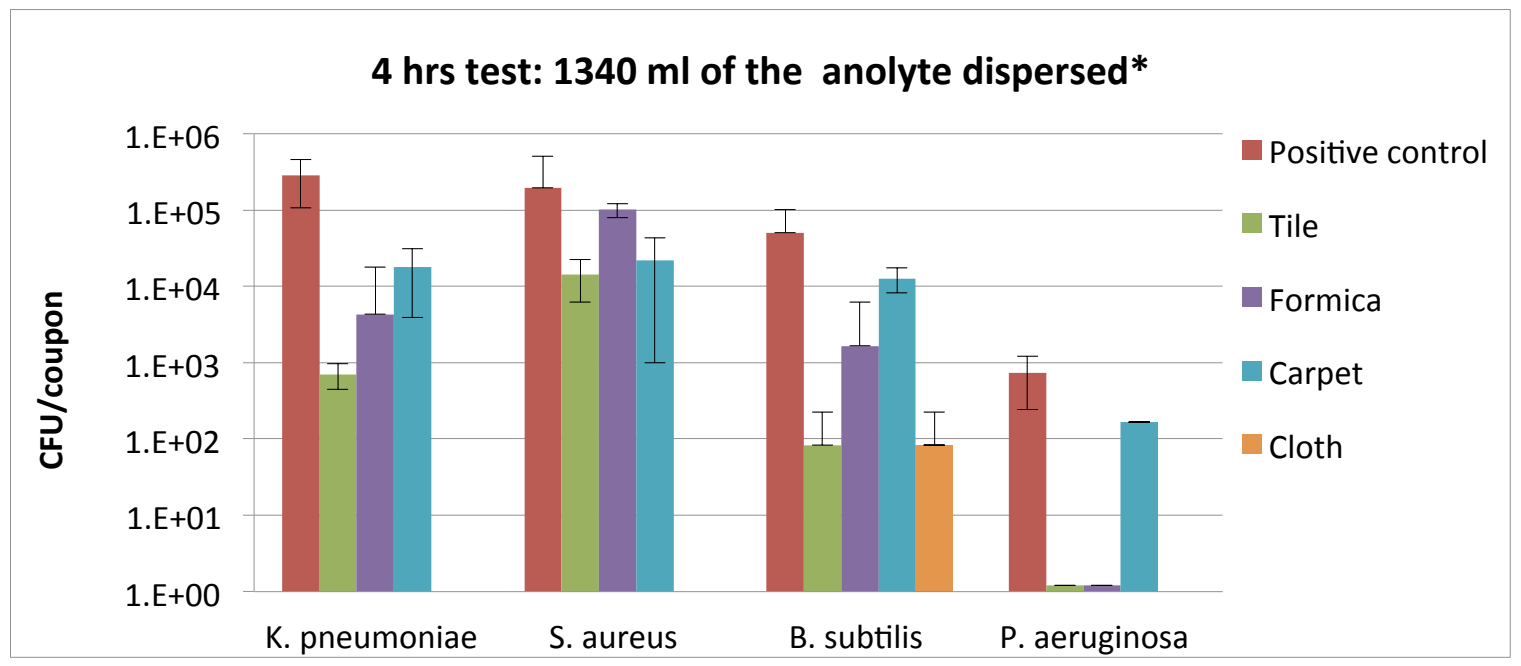

* Test parameters: $\mathrm{NaCl} 0.5 \%$; ROS $0.2 \%$; RH start $31 \%$; $\mathrm{T} 73.2^{\circ} \mathrm{F}$

Figure 5.11. PAEROSOL Performance During Four Hours after Dispersion of $1340 \mathrm{ml}$ of Anolyte

\subsubsection{Test Serial 3}

In this test, the concentration of $\mathrm{NaCl}$ for anolyte production remained unchanged ( 0.5 percent). However, the volume of atomized anolyte was increased to $2 \mathrm{~L}$. To prevent excessive humidity (e.g., colliding of PAEROSOL particles), the $2 \mathrm{~L}$ of anolyte were split in two equal portions. First, PAEROSOL was produced and distributed from $1 \mathrm{~L}$ of anolyte $\left(0.5 \mathrm{ml}\right.$ anolyte/ $\left./ \mathrm{ft}^{3}\right)$ during 16.6 minutes, 
and the coupons remained undisturbed for 2 hours inside of the room after PAEROSOL diffusion. After that, a second portion of anolyte $(1 \mathrm{~L})$ was atomized in 16.6 minutes inside the room, and the coupons remained undisturbed inside the room for the next 2 hours. During the two consecutive anolyte atomizing tests, a total of $1.0 \mathrm{ml}$ of anolyte was atomized per $1-\mathrm{ft}^{3}$ of the test room.

As shown in Figures 5.12 through 5.14, $2 \mathrm{~L}$ of anolyte atomized to PAEROSOL particles $\left(1 \mathrm{ml} / \mathrm{ft}^{3}\right)$ resulted in loss of viability of all microorganisms on all materials, except on the carpet, where only $P$. aeruginosa was reduced below the detection level. Carpet coupons were the most difficult coupons to inoculate. Any attempt to evenly distribute spiking solution over the carpet surface resulted in an agglomeration of $10 \mu \mathrm{l}$ droplets inoculated on the coupons to one big drop on the top of the carpet loops. Therefore, the droplets of spiking suspensions were inoculated into the base of the carpet loops. There was some small but insignificant differences observed in the PAEROSOL efficacy on carpet coupons positioned in different locations. Carpet coupons located in Position 1 (Figure 5.13), under the surgical table in the test room, retained more viable spores than the carpet coupons located in the other positions. However, at the same time, all microorganisms showed loss of viability on the tile coupons, which were positioned side by side with carpet coupons.

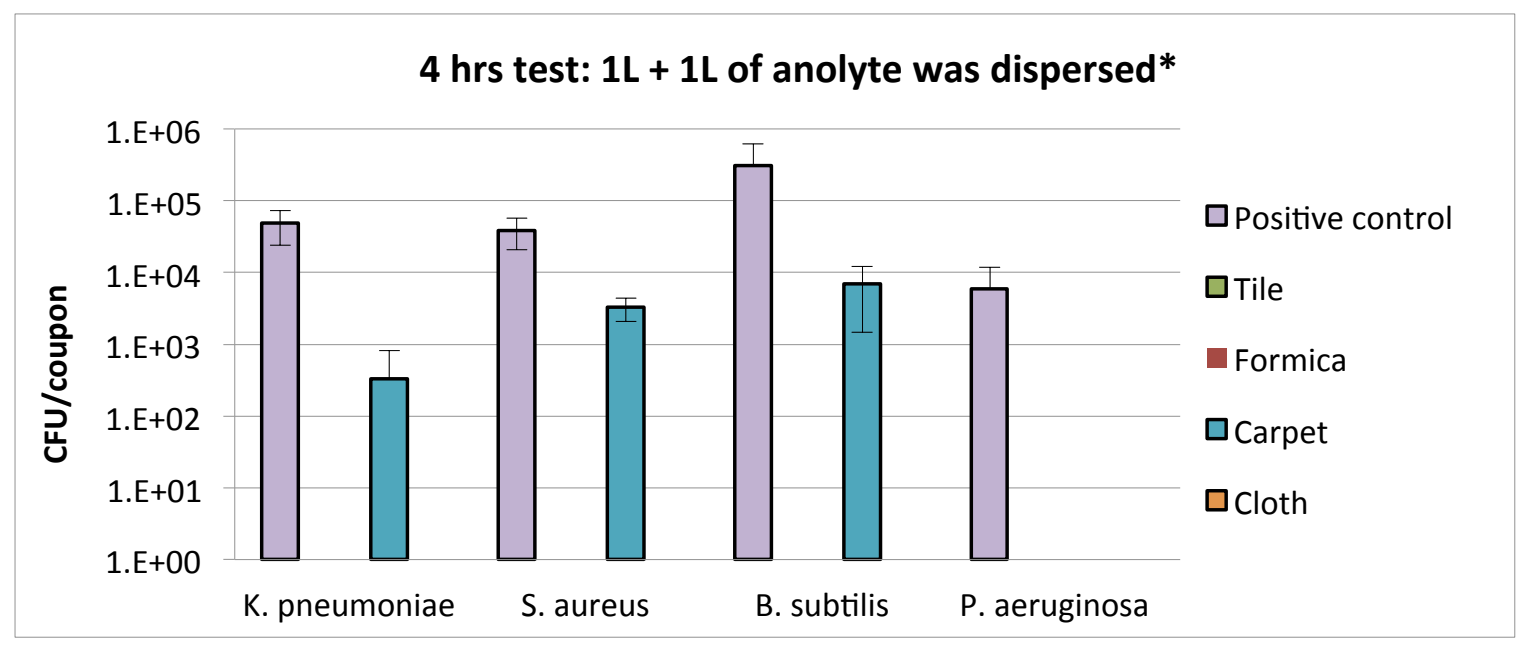

* Test parameters: $\mathrm{NaCl} 0.5 \%$; ROS $0.2 \%$; RH start $21.5 \%$; $66^{\circ} \mathrm{F}$

Figure 5.12. PAEROSOL Performance During Two Consecutive Dispersals of 1 L of Anolyte, with a 2hour Break in Between 


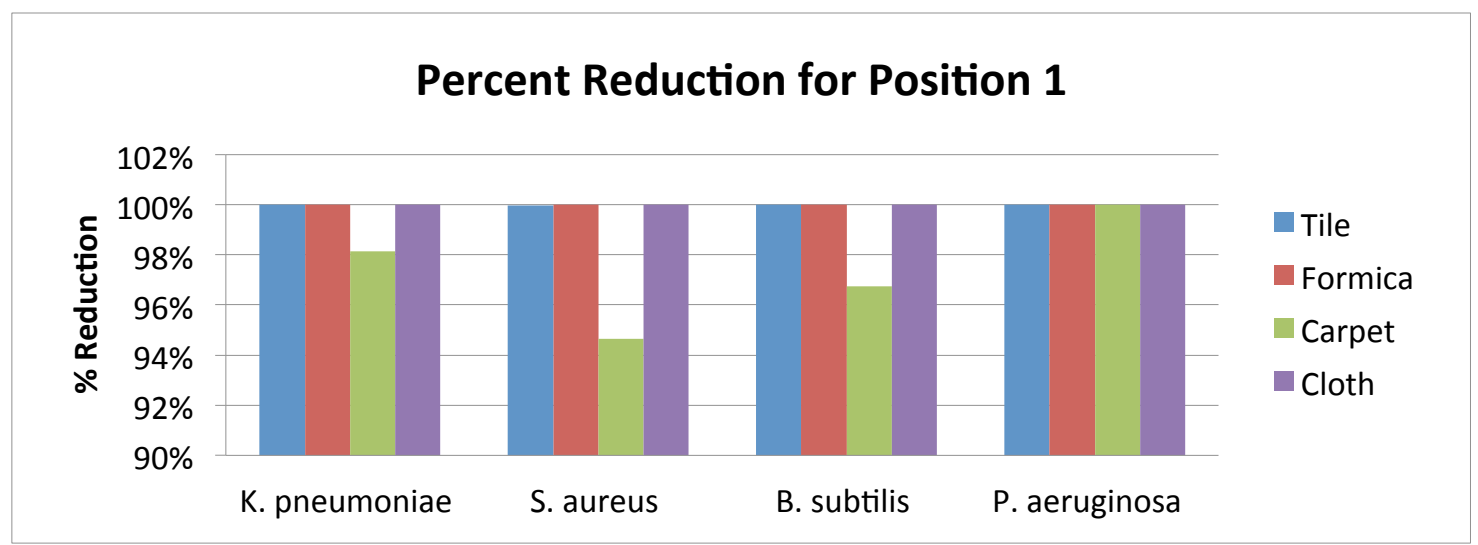

Figure 5.13. Percent Reduction for each Microorganism on each Material in Position 1

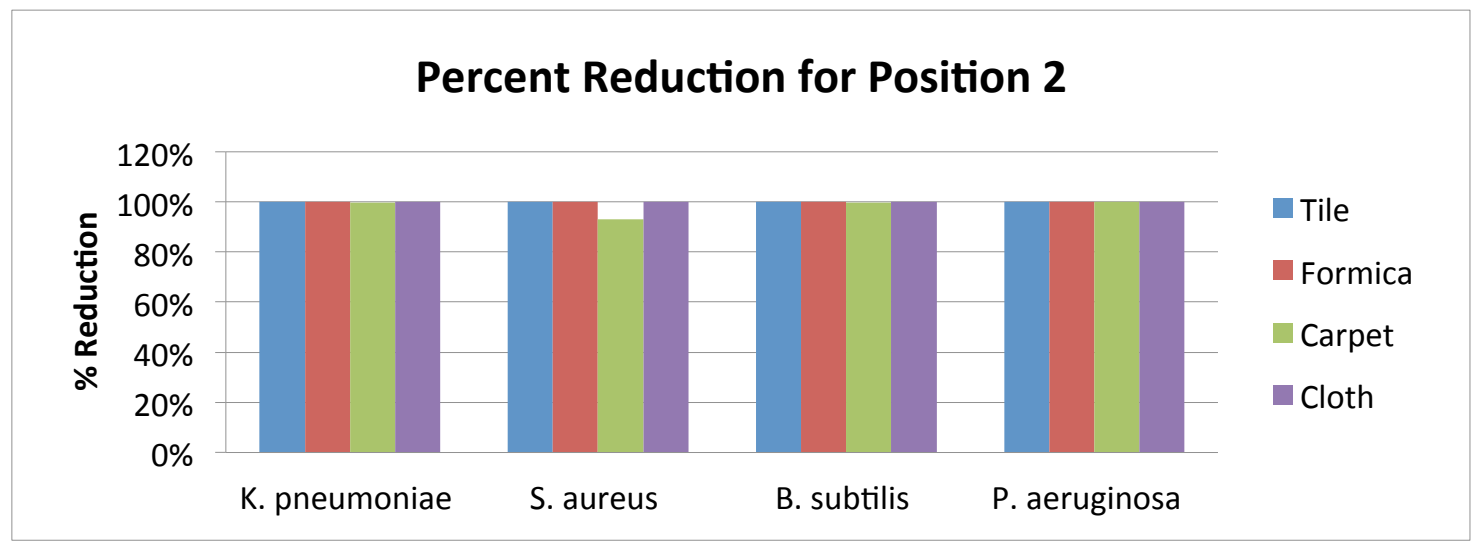

Figure 5.14. Percent Reduction for each Microorganism on each Material in Position 2

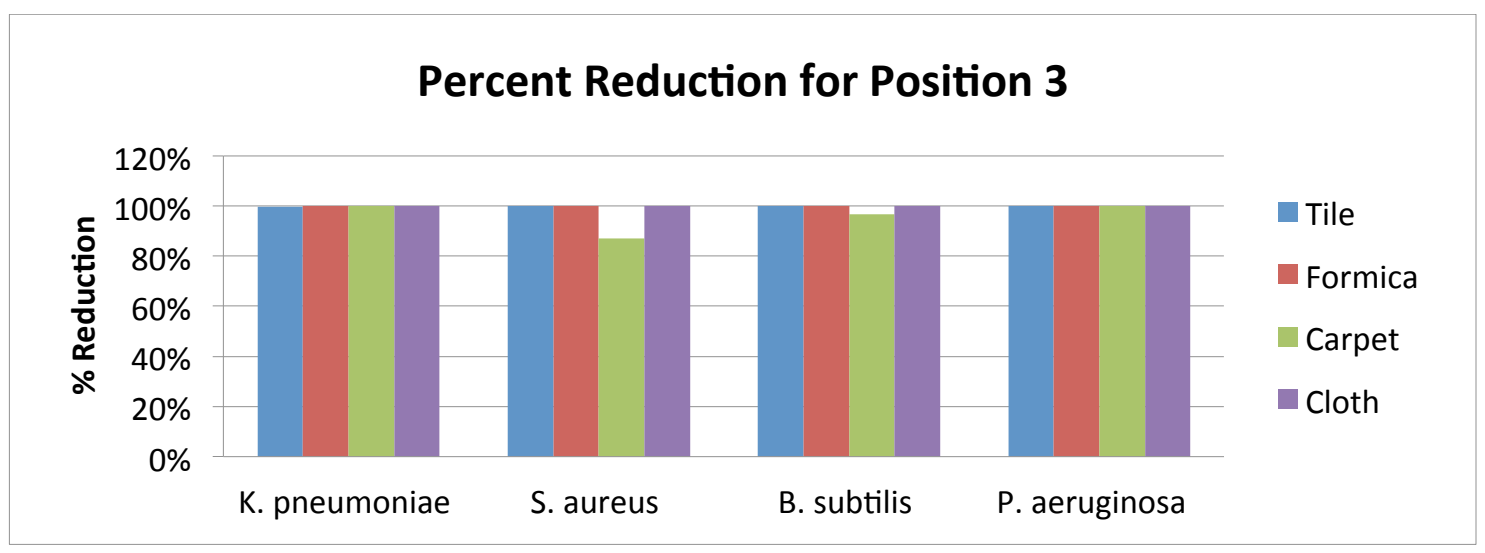

Figure 5.15. Percent Reduction for each Microorganism on each Material in Position 3 


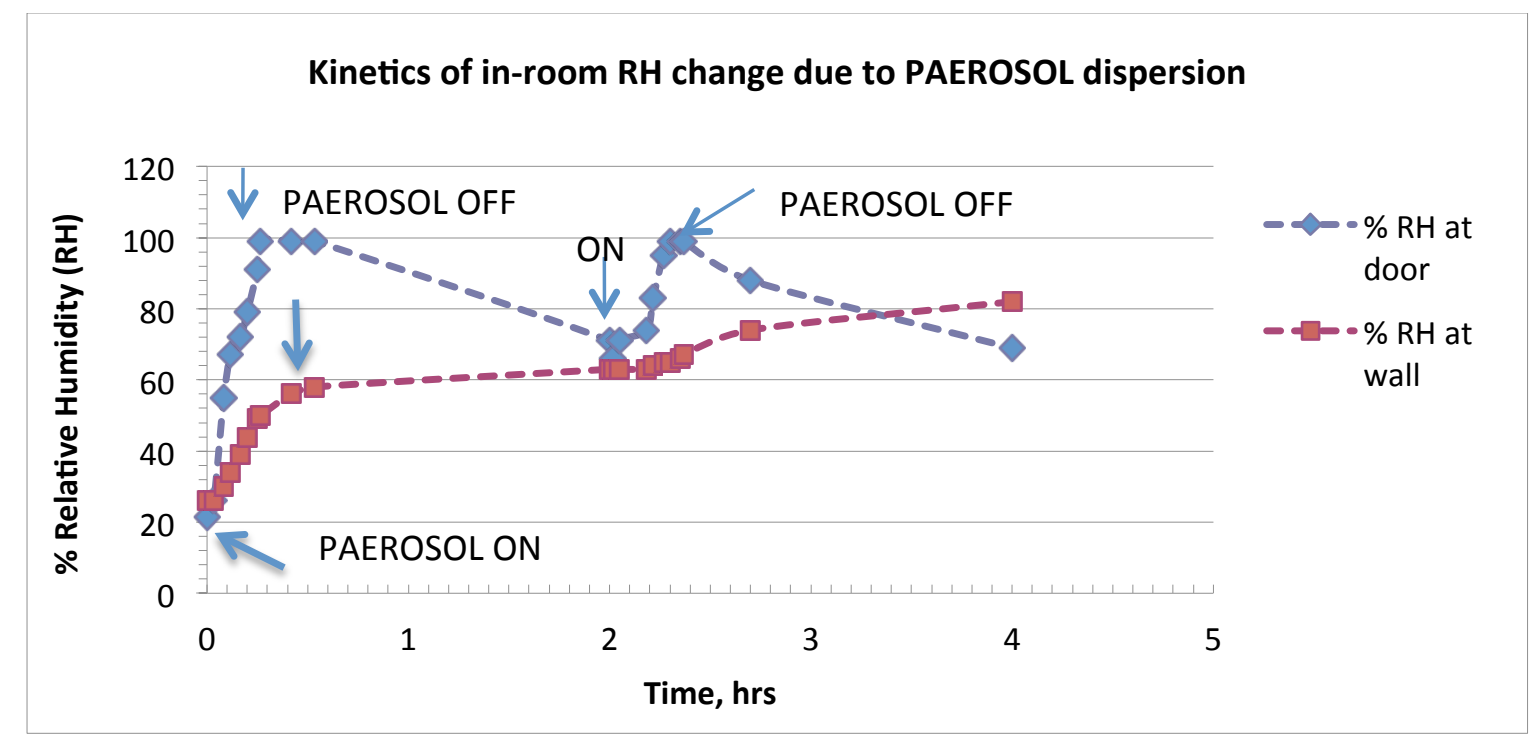

Figure 5.16. Kinetics of In-room RH Change after PAEROSOL Dispersion

Figure 5.16 illustrates variation in the in-room RH as a result of PAEROSOL dissemination. Two wireless RH-temperature sensors (Oregon Scientific THGR122NX Wireless Temperature and Humidity Sensor) were positioned inside the room. One sensor was positioned close to the door and the other was on the opposite side of the room in the corner. Per Figure 5.16, the humidity grew significantly quicker from the side of the door than in the opposite side of the room. After termination of PAEROSOL dissemination, RH near the door gradually dropped down while the RH in the opposite side of the room steadily increased, presumably reflecting continuing air turbulence inside the room. The kinetics of RH increase and decrease after the first and second PAEROSOL disseminations was similar in the door area, whereas the RH on the opposite side of the room slowly increased throughout the test. Obviously, the concentration of PAEROSOL near the door was higher because of the mass exchange through the door. Therefore, the RH near the door was higher than that in the other parts of the room. Interruption of PAEROSOL dissemination was followed by a smaller mass exchange between the room and the hallway. Consequently, the humidity near the door declined to a level approximately the same as the RH in the opposite side of the room.

The accuracy of the sensors used to monitor the temperature and $\mathrm{RH}$ in the real-world environment PAEROSOL study were adequate for the testing environment. The purpose of monitoring RH and temperature in this project was to collect environmental parameters during PAEROSOL dissemination and possibly use it for further PAEROSOL optimization.

It was assumed that PAEROSOL was sucked from under the door gap by the building's HVAC system. Also, a building exit was approximately $5 \mathrm{ft}$ from the door to the test room. This door was opened frequently, but not regularly, by MAMC employees, which also could have contributed to PAEROSOL leaking from the room. $\mathrm{Cl}$ concentration in the hallway was continuously recorded during each PAEROSOL run. Measurable $\mathrm{Cl}$ concentrated in the range of 0.5-0.9 ppm was detected only when the test room door was opened during the test or after the test. At the end of each run, $\mathrm{Cl}$ concentration was also measured in the test room, and it remained in the range of $0.6-1.6 \mathrm{ppm}$. Cl concentration inside the room dropped down to zero, 5 minutes after the door was opened at the end of each run. 


\subsubsection{Test Serial 4}

The next protocol reduced the volume of anolyte atomized to PAEROSOL particles from $1 \mathrm{ml} / \mathrm{ft}^{3}$ (refer to Figure 5.17) to $0.6 \mathrm{ml} / \mathrm{ft}^{3}$. However, the discrete PAEROSOL distribution with a 2-hour break in between and 2 hours post-exposure remained unchanged.

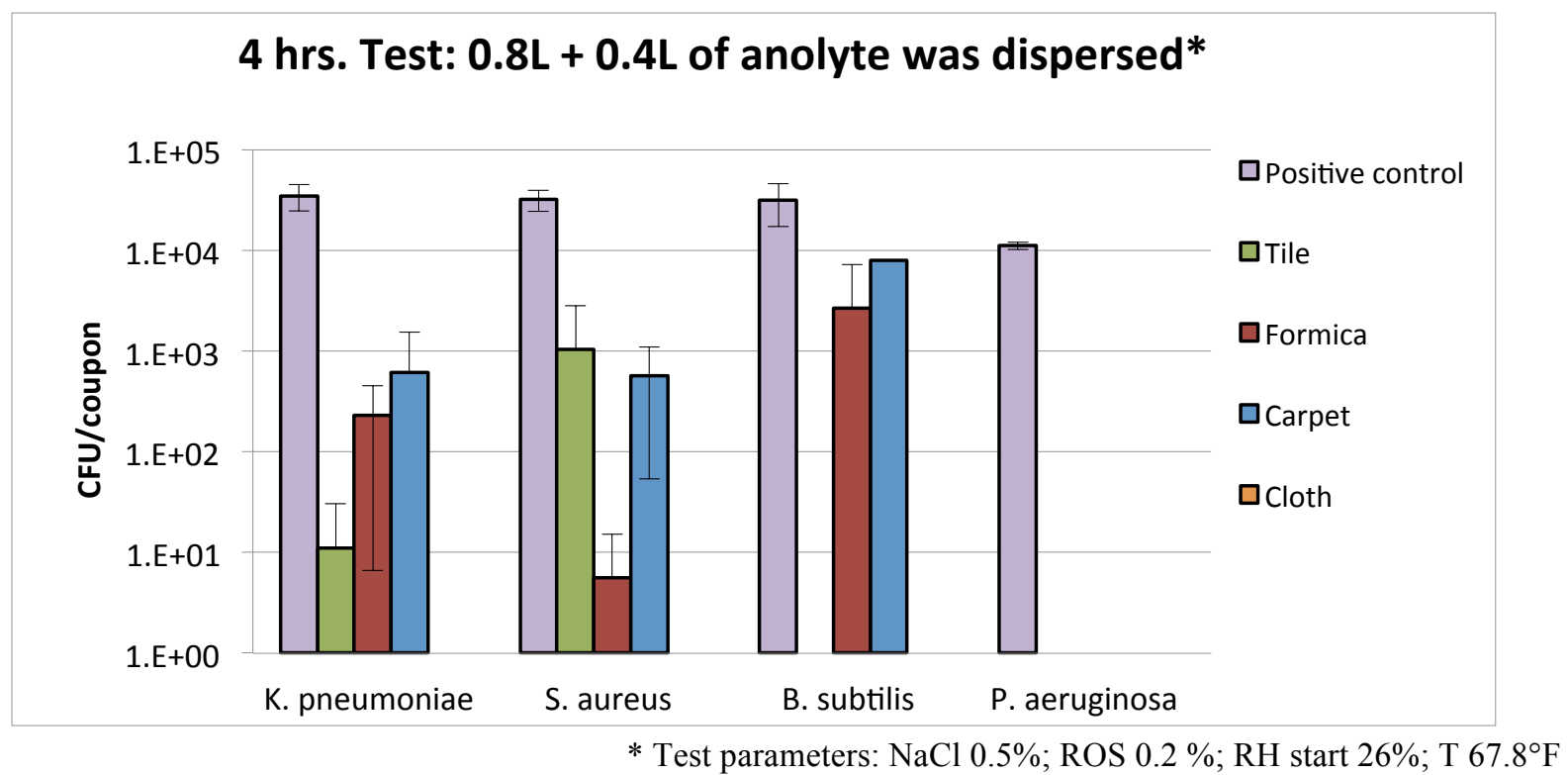

Figure 5.17. PAEROSOL Performance During Two Consecutive Dispersals of $0.8 \mathrm{~L}+0.4 \mathrm{~L}$ of Anolyte with a 2-hour Break in Between

In this test, the first part of PAEROSOL was atomized from $800 \mathrm{ml}$ of anolyte $\left(0.4 \mathrm{ml} / \mathrm{ft}^{3}\right)$ during 13.3 minutes followed by a 2-hour break, during which the coupons remained undisturbed inside the room. The next part of PAEROSOL was atomized from $400 \mathrm{ml}$ of anolyte $\left(0.2 \mathrm{ml} / \mathrm{ft}^{3}\right)$ during 6.7 minutes, followed by 2 hours while the coupons remained undisturbed inside the room. Thus, the total volume of anolyte atomized in this test was $1200 \mathrm{ml}$, providing $0.6 \mathrm{ml}$ of anolyte $/ \mathrm{ft}^{3}$. Comparison of the results presented in Figures 5.12 and 5.17 demonstrate that reduction of dispersed PAEROSOL volume by 40 percent negatively affected PAEROSOL efficacy. Also, a difference between efficacies of decontamination of the coupons positioned in different locations was observed (data not presented).

Of note, PAEROSOL is a self-distributing decontamination method. No additional forces were used in the test room to provide uniformity of PAEROSOL dispersion. The VAG operates by compressed air at an airflow rate at approximately $400 \mathrm{~L} / \mathrm{min}$ at $35-38$ psi. The volume of the PAEROSOL (air blended with micro-droplets of anolyte) is directly proportional to the volume of atomized anolyte when the rate of anolyte atomizing is constant. In this trial, the rate of anolyte atomizing was constant $(60 \mathrm{ml} / \mathrm{min})$ to provide the production of identical PAEROSOL particles in each test (as described in Section 4). Hence, the reduction of the anolyte volume by 40 percent resulted in a 40 percent reduction of the PAEROSOL volume atomized by VAG. Therefore, a total of $8000 \mathrm{~L}$ of PAEROSOL was fumigated during 20 minutes in this unsuccessful test $(13.3 \mathrm{~min}+6.6 \mathrm{~min}$ ) while $13,000 \mathrm{~L}$ of PAEROSOL was fumigated in the successful experiment illustrated in Figure 5.12. It could be assumed that the fumigation of $800 \mathrm{ml}$, and more specifically the fumigation of $400 \mathrm{ml}$ of anolyte, did not provide a satisfactory concentration of 
reactive species or a desirable equilibrium of PAEROSOL across the test room. While this experiment was unsuccessful, the results are important for further PAEROSOL optimization.

A similar experiment was performed with $1.5 \mathrm{~L}$ of the anolyte atomized to PAEROSOL in two portions: $900 \mathrm{ml}$ followed a 2-hour break and another $600 \mathrm{ml}$ of the anolyte followed by 2 hours with the coupons remaining inside the room undisturbed. The concentration of $\mathrm{NaCl}$ for anolyte production remained unchanged ( 0.5 percent). In this experiment, 10,000 L of PAEROSOL was fumigated during 25 minutes, providing $0.75 \mathrm{ml}$ of anolyte per $1-\mathrm{ft}^{3}$. The results of this test (not shown) were less successful than those achieved with atomizing $2.0 \mathrm{~L}$ of anolyte (refer to Figure 5.12), but more successful than was observed with $1.2 \mathrm{~L}$ of the anolyte (Figure 5.17).

\subsubsection{Test Serial 5}

The last variable challenged in this trial was the concentration of $\mathrm{NaCl}$ for the anolyte production.

Figures 5.18 through 5.21 illustrate the results of the test performed in accordance with the protocol used in the experiment shown in Figure 5.12, except that the $\mathrm{NaCl}$ concentration for anolyte production was reduced from 0.5 to 0.25 percent. Concurrently, the ROS concentration in anolyte was decreased from 0.2 to 0.11 percent (as described in Section 4).

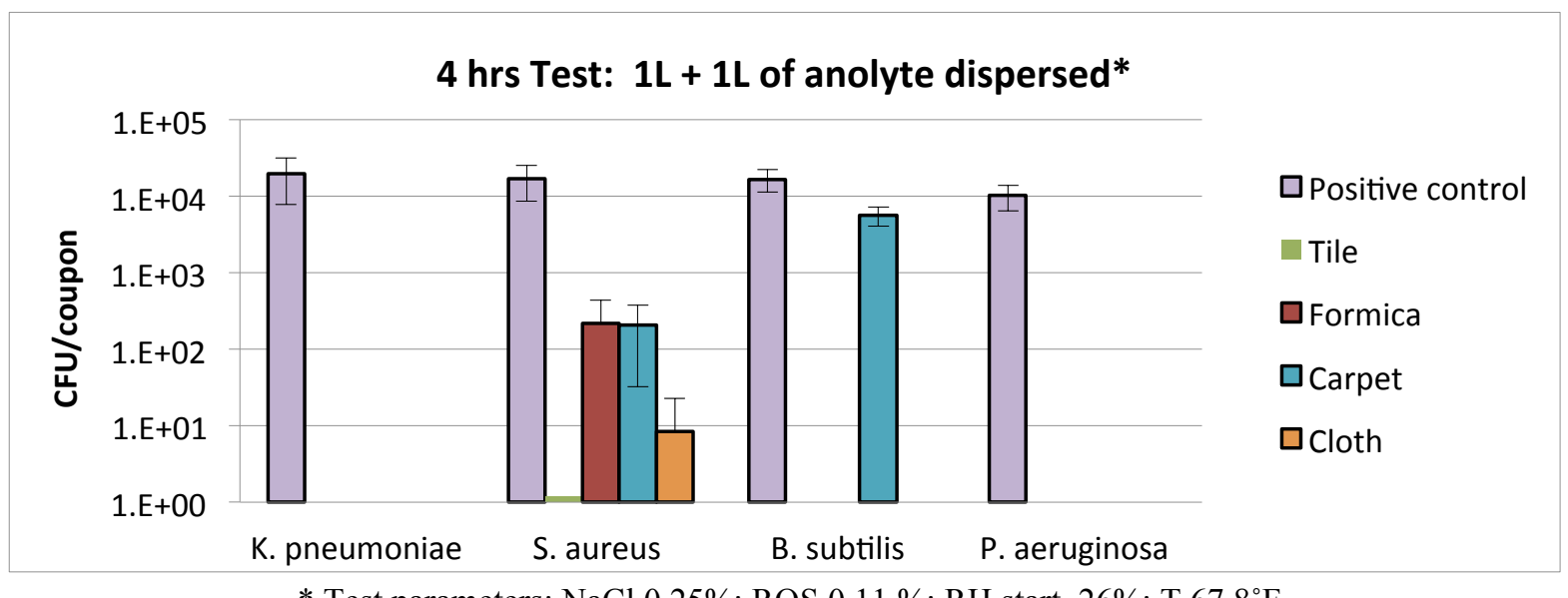

* Test parameters: $\mathrm{NaCl} 0.25 \%$; ROS $0.11 \%$; RH start $26 \%$; $\mathrm{T} 67.8^{\circ} \mathrm{F}$

Figure 5.18. PAEROSOL Performance During Two Consecutive Dispersals of 1.0L + 1.0L of Anolyte (from $0.25 \%$ of $\mathrm{NaCl}$ ) with 2 hours Break in Between 


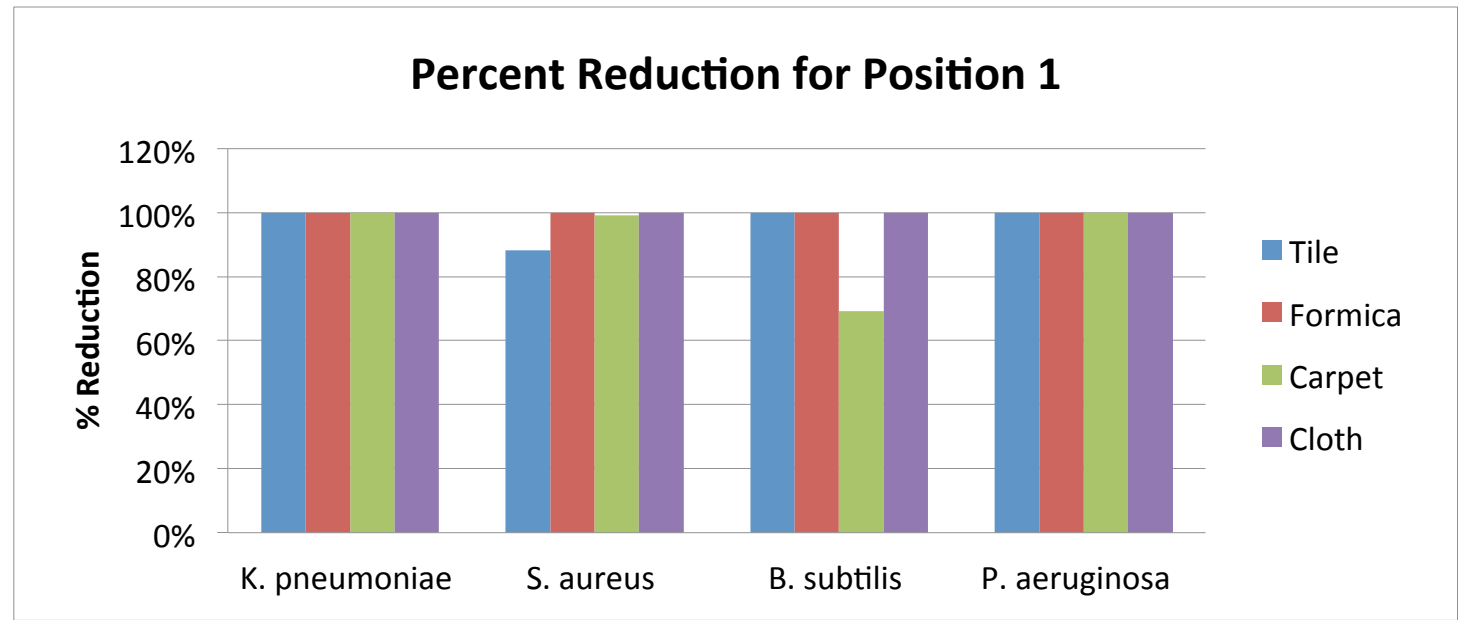

Figure 5.19. Percent Reduction for each Microorganism on each Material in Position 1

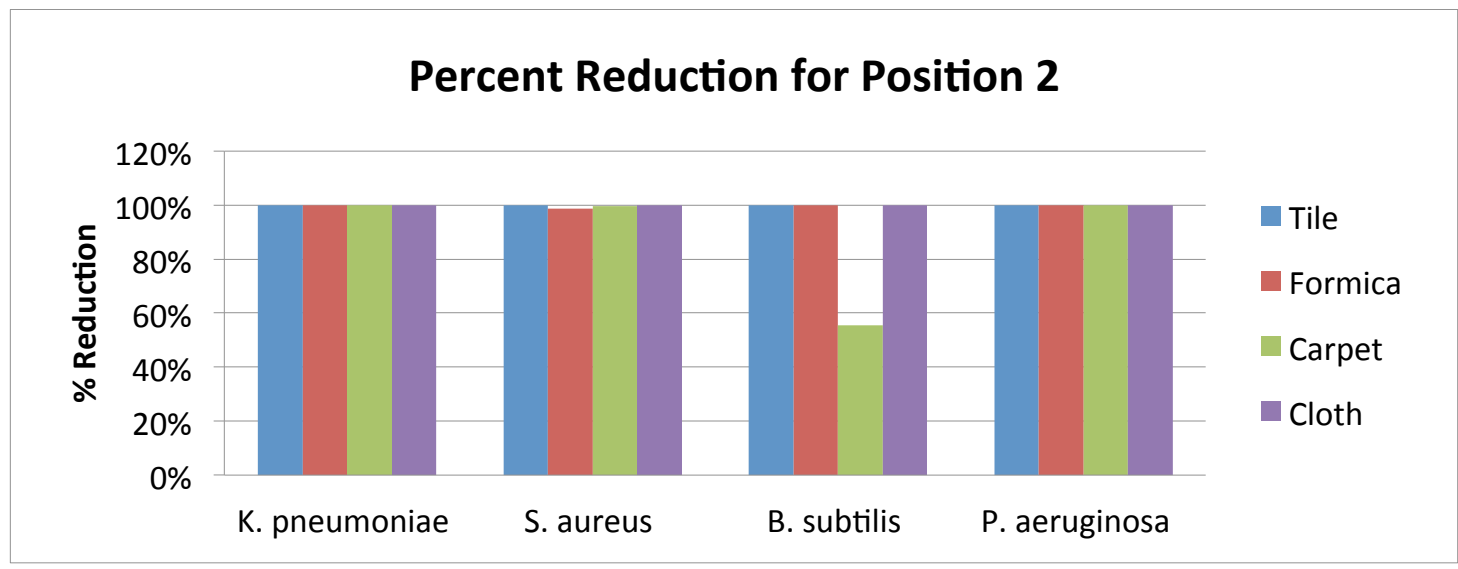

Figure 5.20. Percent Reduction for each Microorganism on each Material in Position 2

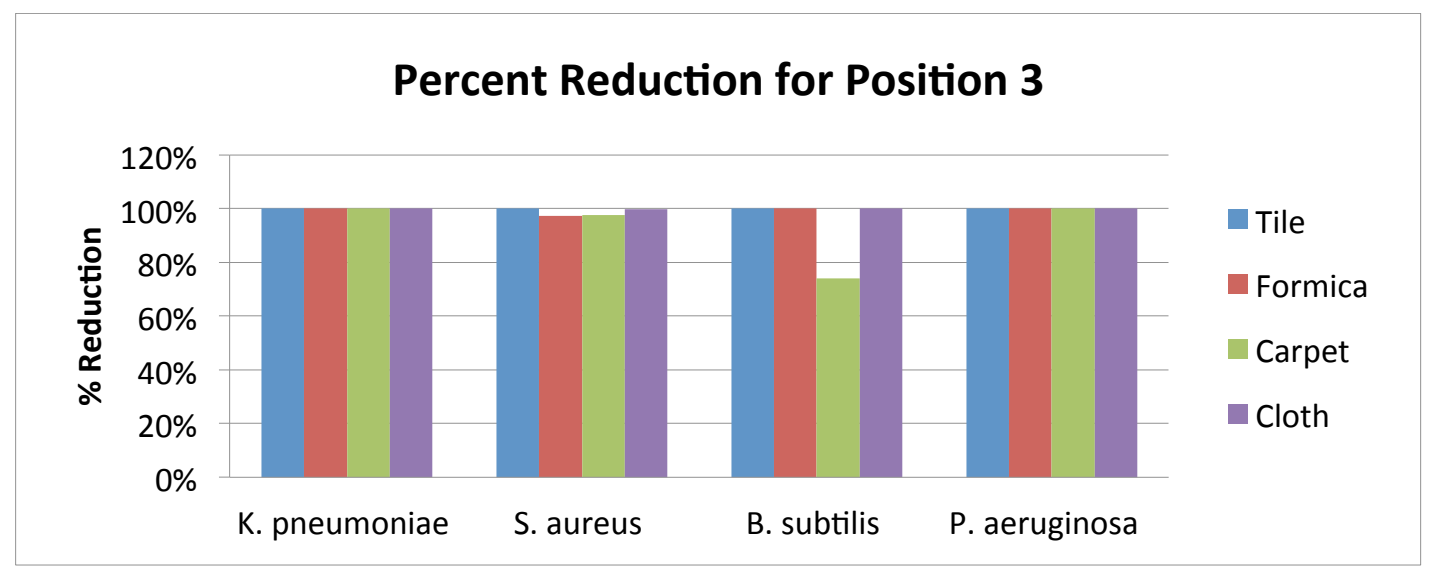

Figure 5.21. Percent Reduction for each Microorganism on each Material in Position 3

Reduction of $\mathrm{NaCl}$ concentration by a factor of two, synchronously reduced ROS concentrations in anolyte (Table 4.1). However, per the results presented in Figures 5.18 through 5.21, the efficacy of PAEROSOL atomized from anolyte containing 0.11 percent of ROS was similar in overall pattern to PAEROSOL atomized from the anolyte containing 0.2 percent of ROS (see Figures 5.12 through 5.16). 
No significant difference was observed in CFU of the microorganisms left on the coupons positioned in different locations. K. pneumoniae and $P$. aeruginosa were not viable on all materials, including carpet. B. subtilis count remained practically unchanged on the carpet, although no viable spores remained on the other materials. Viable S. aureus remained on the carpet, cloth, and Formica, although its CFU dropped down from approximately 20,000 CFU inoculated on carpet, Formica, and cloth coupons to $200 \mathrm{CFU}$ on carpet and Formica, and $10 \mathrm{CFU}$ on cloth. Overall, there was no significant difference observed between efficacies of PAEROSOL prepared from electrochemically activated 0.5 percent $\mathrm{NaCl}$ and 0.25 percent $\mathrm{NaCl}$.

Attempts to reduce the volume of PAEROSOL prepared from anolyte containing 0.11 percent of ROS ( 0.25 percent $\mathrm{NaCl}$ was used) resulted in a significant reduction of PAEROSOL efficacy, as it was observed for different volumes of PAEROSOL atomized from anolyte prepared from 0.5 percent $\mathrm{NaCl}$. As explained, the reduction of utilized anolyte volume synchronously reduced the volume of atomized PAEROSOL. Which of the two factors-less reactive particles per room volume, or a poorer distribution of the particles - should be attributed to the reduction of PAEROSOL efficacy needs to be further investigated.

These results confirmed previously received data concerning complex relationships between the factors that dictated PAEROSOL efficacy in non-controlled environmental conditions.

In the next test, the concentration of $\mathrm{NaCl}$ was further reduced, while the volume of dispersed anolyte remained 2.0 L. PAEROSOL prepared from the 0.125 percent of $\mathrm{NaCl}$ that has been electrochemically activated $(0.05$ percent ROS) was tested in accordance with the following protocol: each $1.0 \mathrm{~L}$ of PAEROSOL dissemination was followed by 2 hours when the coupons remained in the room undisturbed.

The results of the test are illustrated in Figure 5.8. PAEROSOL containing 0.05 percent ROS was not effective as compared to PAEROSOL containing 0.2 percent ROS and 0.1 percent ROS.

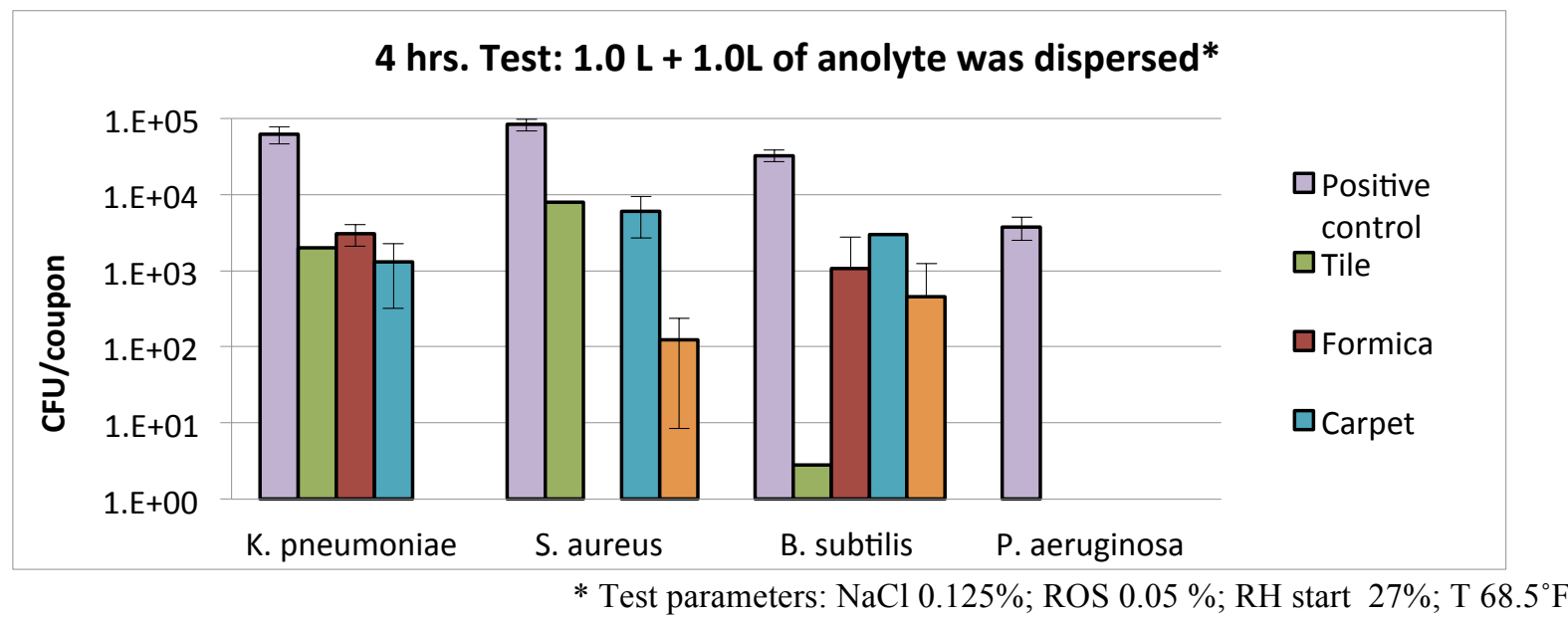

Figure 5.22. PAEROSOL Performance During Two Consecutive Dispersals of 1.0L + 01.0L of Anolyte (from $0.125 \% \mathrm{NaCl}$ ) with 2 Hours Break in Between 


\subsubsection{Test Serial 6}

Reoccurrence of the microorganisms (i.e., an increase in bacterial viability on the plates after extended incubation) on decontaminated materials also characterizes the efficacy of decontamination technology. PAEROSOL-caused reduction in microbial viability is not followed by a later increase in viability. Reoccurrence should not be confused with re-contamination of decontaminated surfaces in real environments, such as hospitals and elsewhere. If the surface was 100-percent decontaminated (no viable organisms left) and no decontaminant residue remained on the surface, recontamination of such surfaces is inevitable for example, because of new contacts with contaminated hands or via aerosol droplets exhaled by a person carrying pathogen, etc. If residues of decontaminant were left on the hospital surfaces, it could control recontamination for a certain amount of time. However, these residuals may also contribute to the appearance of chemical-resistant strains of microorganisms (Mc Cay et al. 2010).

Reoccurrence of microorganisms on decontaminated surfaces means that the same microorganisms that did not appear to be viable, became viable and grew/appeared on the decontaminated surfaces after an extended period of time. Why is this an issue, specifically in validation of decontaminant efficacy in a coupon-based study? Because, microorganisms that were air-dried on the surfaces could be temporarily shocked or slightly damaged prior to and resulting from the PAEROSOL treatment. Therefore, the inability of some (or all) cells to be cultured immediately after decontamination may provide falsenegative results. Also, if disinfectant was left on the surface, it could temporarily prevent microorganisms' reproduction and contribute to the reduction of viable microorganisms. Usually, in coupon-based validation, disinfectants are neutralized on the coupons to avoid such bias. One of the advantages of PAEROSOL application is that it does not leave germicidally active decontaminant residue on the surfaces and does not require residue neutralization. What exactly is left on the surfaces after PAEROSOL application will be discussed in Test Serial 8.

Here the results confirming no evidence of post-PAEROSOL treatment of microorganisms (no organisms' reoccurrence on the coupons after exposure to PAEROSOL) will be described. Previously, we have demonstrated that there was no reoccurrence of B. cereus spores and Salmonella eneteritidis vegetative cells after 2 weeks following their decontamination on glass and some other materials. In this test, the observation of reoccurrence of the microorganisms on the coupons after PAEROSOL application was limited to 2 days because of time and budget constraints.

The test was performed using the protocol described in Test Serial 3 and illustrated in Figures 5.12 through 5.16. Multiple coupons of each material contaminated with each microorganism were positioned

as described for all other tests. After decontamination completion, all coupons were withdrawn from the test room in closed Petri dishes. The first set of decontaminated coupons and positive control coupons (each microorganism on each type of material but not decontaminated) was extracted and processed as described. The following 2 days, sets of decontaminated coupons were extracted and processed. Limited dilutions were done, and undiluted extracts also were plated. The results of the test are presented in Figures 5.23 through 5.26. 


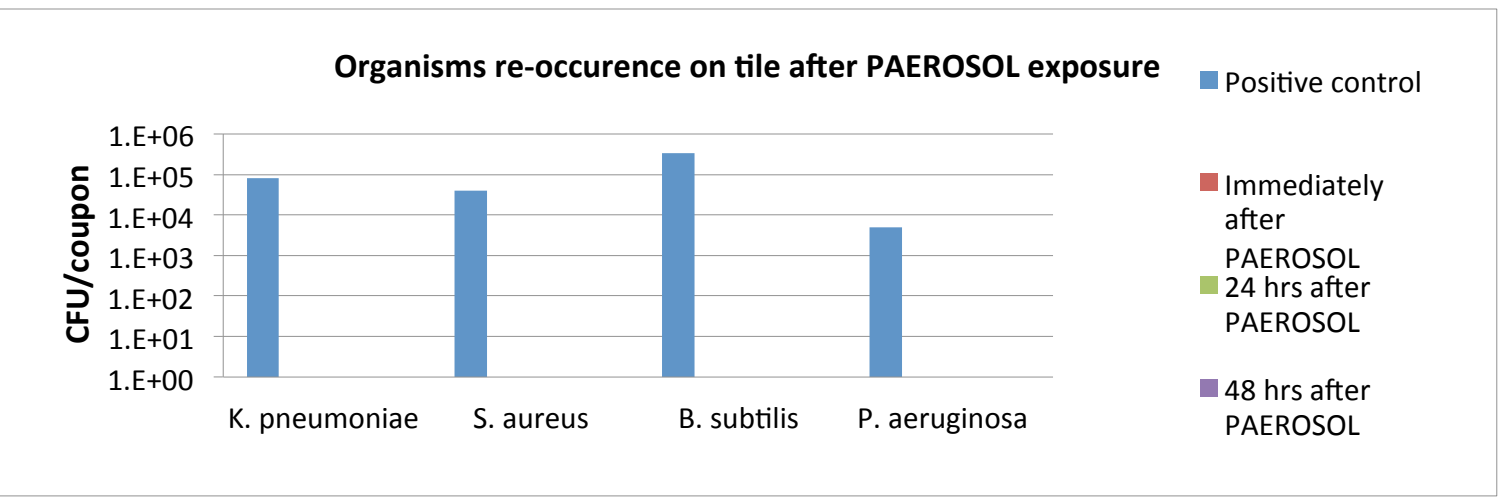

Figure 5.23. Organisms' Reoccurrence on Tile after PAEROSOL Exposure (Decontamination parameters: $\mathrm{NaCl}=0.5 \%$; $\mathrm{ROS}=0.21 \%$; $\mathrm{RH}$ start $=32 \%$; and $\mathrm{T}=69.5^{\circ} \mathrm{F}$ )

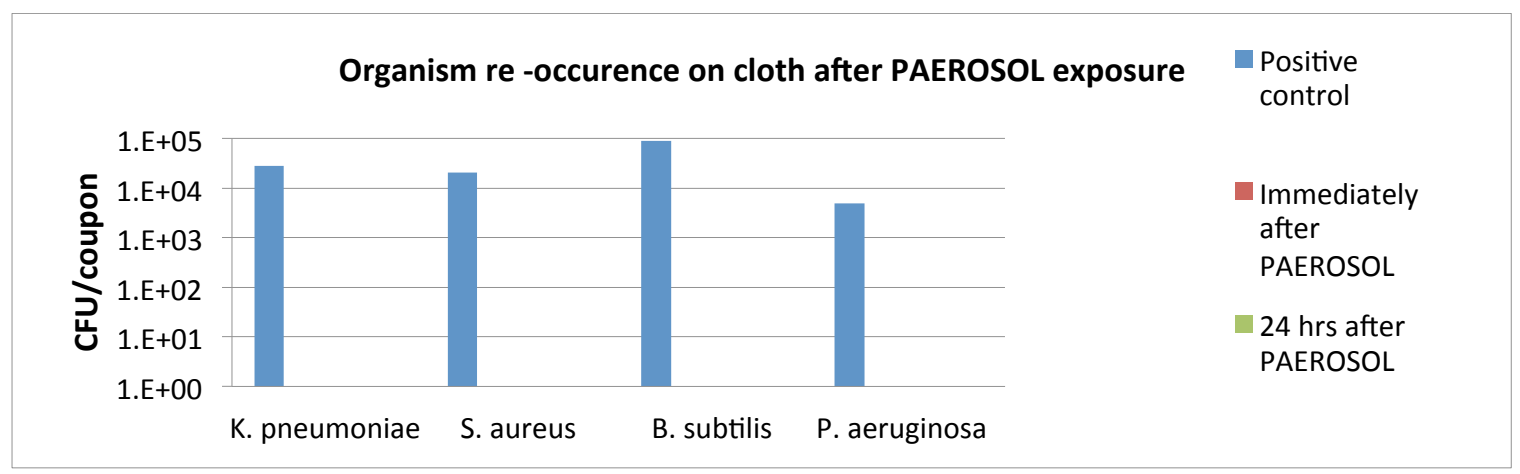

Figure 5.24. Organisms' Reoccurrence on Cloth after PAEROSOL Exposure (Decontamination parameters: $\mathrm{NaCl}=0.5 \%$; ROS $=0.21 \%$; $\mathrm{RH}$ start $=32 \%$; and $\mathrm{T}=69.5^{\circ} \mathrm{F}$ )

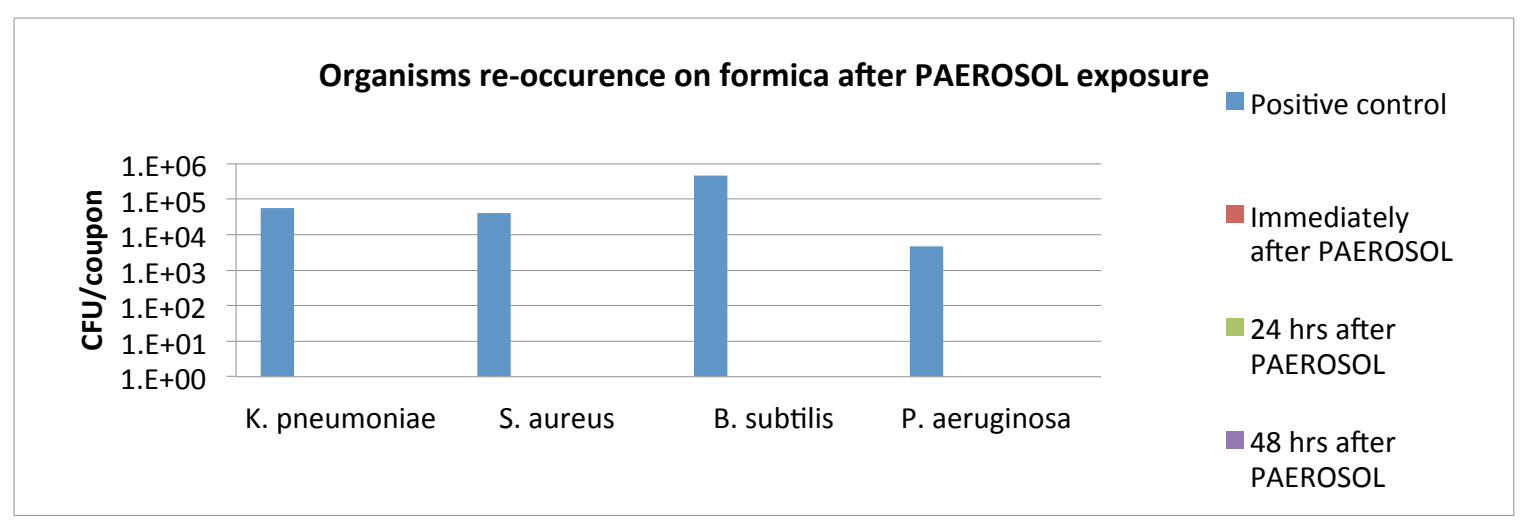

Figure 5.25. Organisms' Reoccurrence on Formica after PAEROSOL Exposure (Decontamination parameters: $\mathrm{NaCl}=0.5 \%$; $\mathrm{ROS}=0.21 \%$; $\mathrm{RH}$ start $=32 \%$; and $\mathrm{T}=69.5^{\circ} \mathrm{F}$ ) 


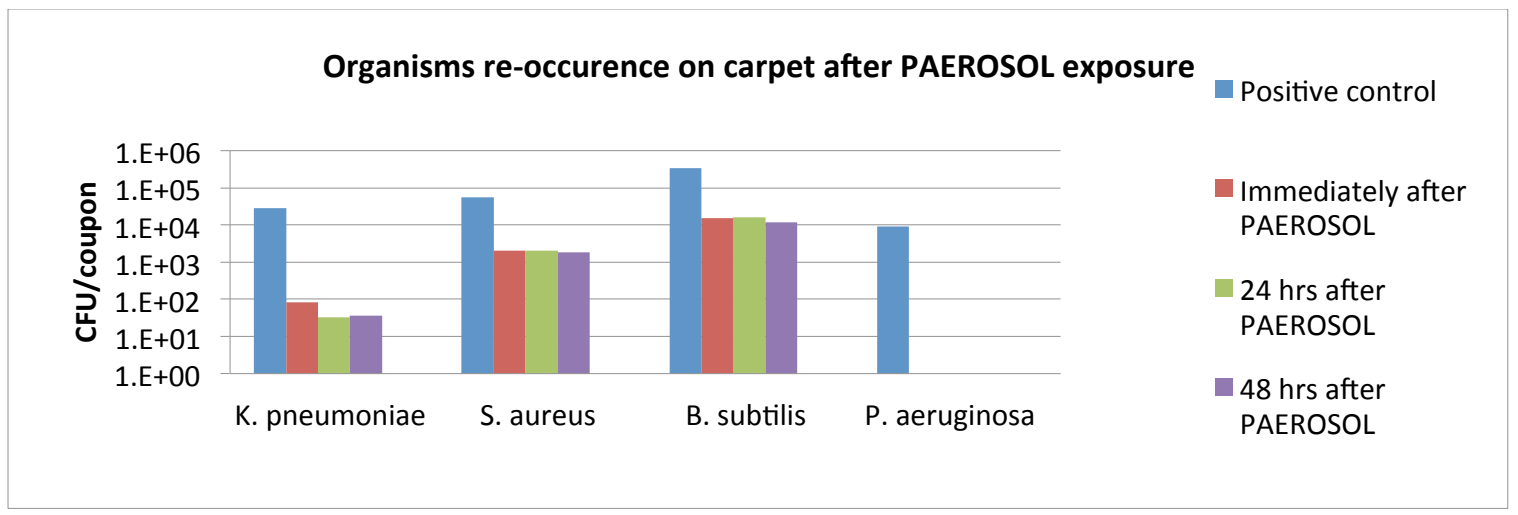

Figure 5.26. Organisms' Reoccurrence on Carpet after PAEROSOL Exposure (Decontamination parameters: $\mathrm{NaCl}=0.5 \%$; ROS $=0.21 \%$; $\mathrm{RH}$ start $=32 \%$; and $\mathrm{T}=69.5^{\circ} \mathrm{F}$ )

As depicted by Figures 5.23 through 5.26, all microorganisms on all materials showed no viability when decontaminated with PAEROSOL, except on carpet coupons. No reoccurrence of organisms on decontaminated materials was observed. On carpet coupons, the number of viable organisms remained unchanged during 48 hours compared to the number of viable organisms that survived PAEROSOL exposure.

\subsubsection{Test Serial 7}

It was previously demonstrated that atomized $\mathrm{NaCl}$ solutions that have not been electrochemically activated had no germicidal activity. We confirmed these results during the trial in MAMC. A control test was performed to compare germicidal efficacy of the microaerosol atomized from 0.5 percent $\mathrm{NaCl}$ that was not electrochemically activated with PAEROSOL. The experimental protocol was identical to that described in Test Serial 5. After experimental completion, the coupons were processed. CFU reduction was not observed for all tested organisms on all tested materials.

\subsubsection{Test Serial 8}

After the PAEROSOL microdroplets are desiccated, the only residue remaining on the horizontal surfaces was $\mathrm{NaCl}$ precipitate. The quantity of precipitated $\mathrm{NaCl}$ after PAEROSOL dispersion depends on: 1) $\mathrm{NaCl}$ concentration used for anolyte production, 2) efficacy of the electrochemical module, and 3) the volume of PAEROSOL dispersed (volume of anolyte atomized to PAEROSOL particles). Because $\mathrm{NaCl}$ is known to cause corrosion, the concentration of $\mathrm{NaCl}$ solution for PAEROSOL application and PAEROSOL volume was varied in this trial.

Electrochemical activation in modern FEM-type reactors claims 90-98 percent salt conversion. However, practical data often differs from the theoretical data. Two different volumes of anolyte produced from 0.5 percent and 0.25 percent $\mathrm{NaCl}$ were disseminated inside the room to collect experimental data on $\mathrm{NaCl}$ precipitation on horizontal surfaces in the test room. Before the test, 140-mm Petri dishes without lids were positioned in the same locations designated for coupons and in other places in the test room (see Figure 4.2). For the first test, $1.6 \mathrm{~L}$ of analyte produced from 0.25 percent $\mathrm{NaCl}$ was atomized to PAEROSOL. For the second test $2.3 \mathrm{~L}$ of analyte produced from 0.5 percent $\mathrm{NaCl}$ was 
atomized to PAEROSOL. The standard protocol with 2-hour intervals between PAEROSOL

dissemination was implemented. At the completion of the 4 hours, the dishes were collected, and each dish was rinsed with $10 \mathrm{ml}$ of Milli-Q water. Conductivity in each rinsed solution was analyzed, and the concentration of $\mathrm{NaCl}$ precipitated in each dish was calculated using a calibration plot. Figure 5.10 shows the concentration of precipitated $\mathrm{NaCl}$ recalculated per $1-\mathrm{m}^{2}$ of horizontal surface in the indicated areas.

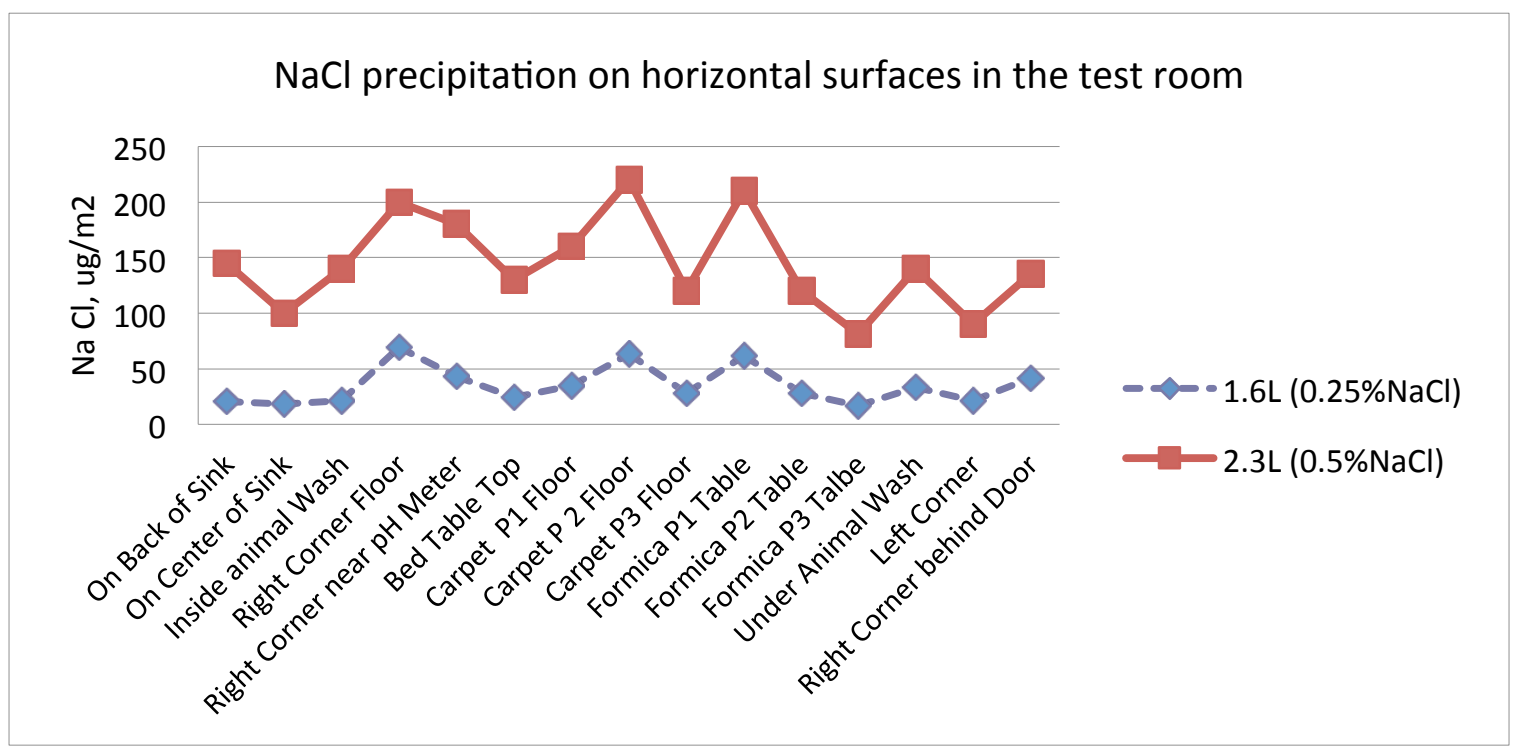

Figure 5.27. $\mathrm{NaCl}$ Collected in the Test Room after PAEROSOL Fumigation

PAEROSOL atomized from $1.6 \mathrm{~L}$ of the anolyte produced from 0.25 percent $\mathrm{NaCl}$, left residual $\mathrm{NaCl}$ on horizontal surfaces in the range of $20 \mu \mathrm{g}$ to $70 \mu \mathrm{g}$ per $1 \mathrm{~m}^{2}$; PAEROSOL atomized from $2.3 \mathrm{~L}$ of the anolyte produced from 0.5 percent $\mathrm{NaCl}$ left residual $\mathrm{NaCl}$ in the range of $120 \mu \mathrm{g}$ to $180 \mu \mathrm{g}$ per $1 \mathrm{~m}^{2}$. The amount of the salt used for $2.3 \mathrm{~L}$ anolyte production $(11.5 \mathrm{~g})$ was approximately three times higher than the amount of the salt used for $1.6 \mathrm{~L}$ anolyte production of $(4 \mathrm{~g})$ (Table 5). In the same time, the amount of deposited salt from the $2.3 \mathrm{~L}$ of atomized anolyte produced from the 0.5 percent $\mathrm{NaCl}$ was approximately four times higher, compared to deposited salt from the $1.6 \mathrm{~L}$ of anolyte produced from the 0.25 percent $\mathrm{NaCl}$. It could be concluded that 0.25 percent $\mathrm{NaCl}$ was more effectively electrolyzed and therefore less non-electrolyzed $\mathrm{NaCl}$ remained in the anolyte. However, we do not have enough data to provide such an explanation. One can see that the deposition was not uniform and it could be attributed to non-homogenous convection, though more data are required to understand this phenomenon.

Table 5.2. $\mathrm{NaCl}$ Deposited Compared to $\mathrm{NaCl}$ Used in the Anolyte

\begin{tabular}{|c|c|c|c|}
\hline Anolyte Volume & NaCL (\%) & Total NaCl used for anolyte production (g) & $\begin{array}{c}\text { Ave. NaCl Deposited } / \mathrm{m}^{2} . \\
\mu \mathrm{g}\end{array}$ \\
\hline 1.6 & 0.25 & 4.0 & $33.93 \pm 17$ \\
\hline 2.3 & 0.5 & 11.5 & $144.73 \pm 42.33$ \\
\hline
\end{tabular}




\subsubsection{Test Serial 9}

PAEROSOL efficacy was qualitatively evaluated for decontamination of microbial cells and spores freshly plated on TSA and TSA with 5-percent sheep blood. TSA and especially TSA with 5-percent sheep blood are rich nutrition media containing high concentrations of proteins and other organic compounds. Thus, in this test, PAEROSOL efficacy was challenged with a high load of organics during the PAEROSOL treatment of the room. Approximately $1 \times 10^{4}$ of freshly grown K. pneumonia and $S$. aureus vegetative cells, and B. subtilis spores, were inoculated in duplicate on TSA or TSA with 5 percent sheep blood and positioned inside the room at the locations pre-determined for the coupons (see Figure 4.2). The lids were removed from the Petri dishes. In addition to that, Petri dishes inoculated with each culture were left covered. Positive control Petri dishes were prepared for each culture in duplicate and left at room temperature in the BSC. PAEROSOL containing 0.2 percent ROS (corresponds to 0.5 percent $\mathrm{NaCl}$ used for anolyte production) was dispersed inside the test room. PAEROSOL fumigation was followed by 2 -hour breaks when dishes remained undisturbed inside the room. Overall, the dishes with inoculated microorganisms were exposed to PAEROSOL over a period of four hours. After that, the dishes were covered by lids and placed in an incubator at $37^{\circ} \mathrm{C}$ for 24-48 hours. Simultaneously, positive control dishes were transferred from the BSC and also placed in an incubator for $24-48$ hours at $37^{\circ} \mathrm{C}$. After an 18-24 hour period, non-countable quantities of the colonies (a converging "lawn" of undistinguishable colonies) were observed on all dishes not exposed to PAEROSOL. There were no microbes growing on the uncovered dishes exposed to PAEROSOL after 48 hours. A very high growth inhibition was observed on the dishes that were covered by lids during exposure of the room to PAEROSOL (Figure 5.11). This confirms our hypothesis that PAEROSOL germicidal efficacy is achieved via ROS offgassing of PAEROSOL particles during the process of particle drying. That is, if ROS offgassing was not an important factor, high growth inhibition would not have been observed on the dishes that were covered by lids (Figure 5.28).
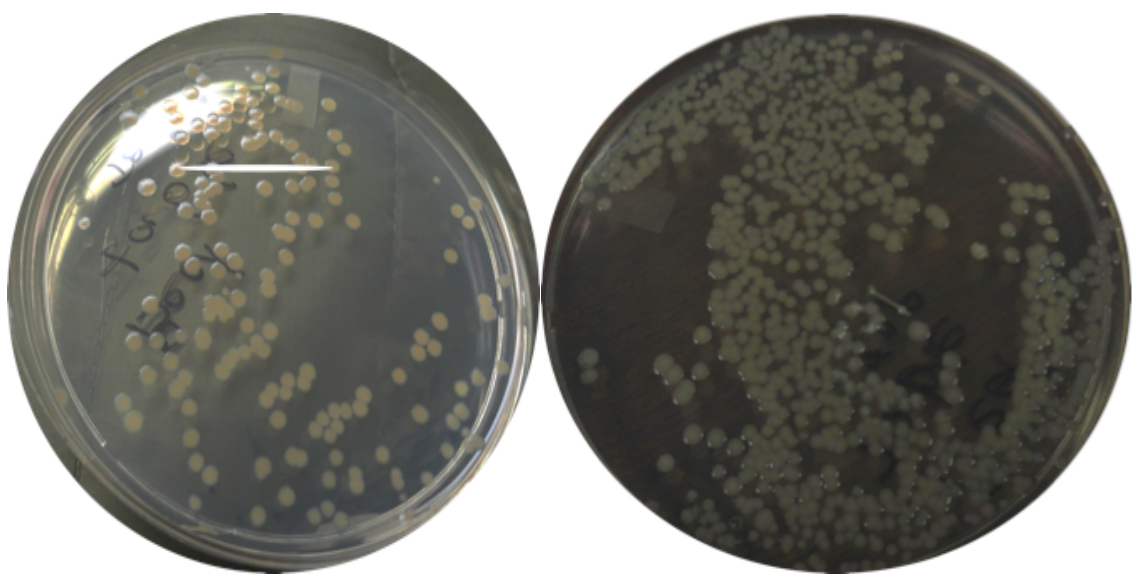

Figure 5.28. K. pneumoniae (left) and S. aureus (right) in Petri Dishes Covered with Lids During 4 hours of PAEROSOL Treatment

In the case where PAEROSOL was fumigated from the anolyte containing 0.11 percent of ROS ( 0.25 percent $\mathrm{NaCl}$ to produce anolyte), culture growth was observed in the uncovered dishes exposed to PAEROSOL, but it was almost negligible compared with the positive control dishes not exposed to 
PAEROSOL (Figure 5.29). Overall, the test results demonstrated PAEROSOL efficacy to decontaminate microorganisms in the presence of a high organic load.
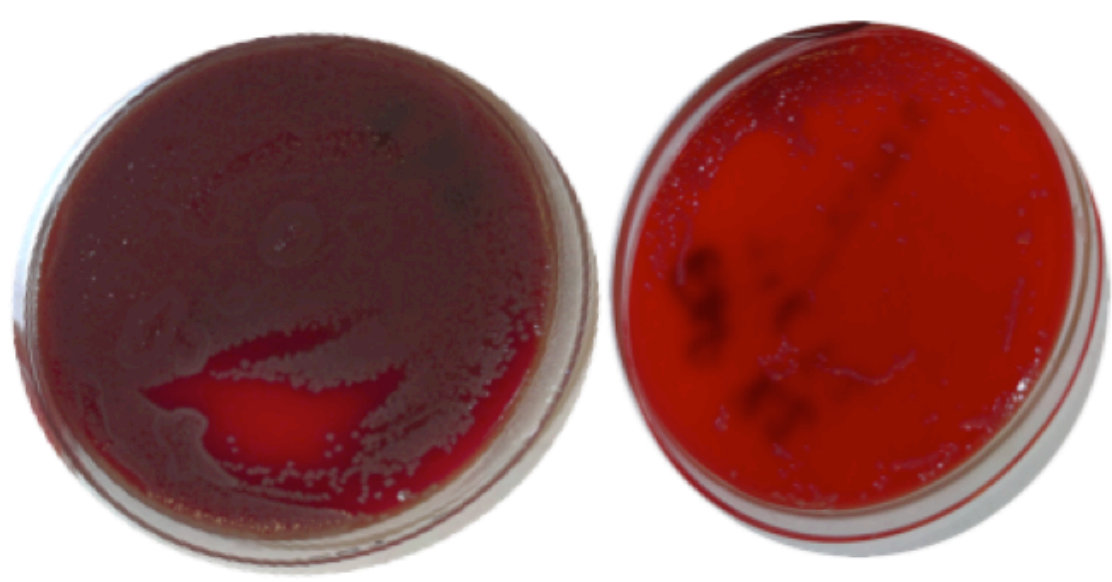

Figure 5.29. K. pneumoniae (Inoculated on TSA with $5 \%$ Sheep Blood) in the Control (left) and in the Petri Dish Exposed to PAEROSOL for 4 Hours

\subsubsection{Damage to Coupons, Inanimate Surfaces and Test Room Equipment}

After each PAEROSOL test, the test coupons were visually inspected by comparing color, reflectivity, and apparent roughness of the decontaminated coupon surfaces with control coupons that had not been exposed to PAEROSOL. No obvious damage (e.g., change in surface texture, color) or visible change was observed to any of the test coupons during PAEROSOL evaluation.

After PAEROSOL test completion, inanimate surfaces in the room were inspected, including walls, door, ceiling, floor, furniture, faucets, electronic equipment, posters, plastic containers, etc. No visual deterioration to the surfaces was observed, except for some small/light rusting spots on a metal paper towel dispenser mounted on the wall, and on the table legs (both were not stainless steel). Whether the rusting was caused by regular PAEROSOL application remains uncertain because we may have overlooked them during initial inspection of the room before testing. We also noticed that the schematics drawn with markers on the floor and on the lab table for coupon placement, gradually faded during the 4 months of the test. It is uncertain whether it was a normal time-related discoloring, or a PAEROSOL bleaching effect. No discoloring or other damage was observed on the fabric and plastic-metal frame of the chair in the room through the trial.

Electronic and electrical equipment that remained in the test room was limited to a $\mathrm{pH}$-meter with multiple probes, a peristaltic pump, a STEL Electrolyzer, timers, humidity/temperature sensors, an electronic clock, room ceiling lights, and a surgical ceiling lamp. Except for the surgical lamp, all other devices were regularly used in the tests and therefore, no special inspection of their operational fitness was necessary. At the end of the test the surgical lamp was fully operational. 


\subsubsection{Observations and Ease of Use}

Quantitative assessment was done to document the ease of PAEROSOL preparation and application. Reliability and reproducibility of the STEL Electrolyzer and the VAG PAEROSOL generator were monitored during each experiment.

The parameters of STEL electrolyzer operation were attenuated in the beginning of the test to satisfy the required production of anolytes. No additional adjustment of parameters was required through the test. Twenty to twenty-five minutes were required to prepare 2.5 liters of anolyte.

VAG productivity was adjusted to atomize microaerosol with the particles diameter in the range of $0.5 \mu \mathrm{m}-10 \mu \mathrm{m}$ at the beginning of the test. No additional adjustment of VAG parameters was required throughout the test. 



\subsection{Performance Summary}

PAEROSOL, a semi-dry, micro-aerosol decontamination technology, was evaluated against common HAI in a non-human subject trial within a hospital setting at MAMC. Upon MAMC's recommendation, PAEROSOL germicidal efficacy was validated against Klebsiella pneumoniae, Pseudomonas aeruginosa, and Staphylococcus aureus - bacteria causing significant percent of the HAIs - and Bacillus subtilis spores (as a simulant for Clostridium difficile spores), inoculated on 1-in ${ }^{2}$ coupons of four materials from MAMC settings, at the concentration range between $10^{3}$ to $10^{5} \mathrm{CFU}$ per coupon.

A protocol for PAEROSOL application that met MAMC expectations in the given MAMC test room conditions provided a 3-5 $\log _{10}$ per coupon reduction (to the detection limit) in viable microbial cells and spores, which were initially inoculated on the coupons of tile, Formica, and fabric. On coupons of carpet, the log reduction depended on the microbe type and was in the range $-1-4 \log _{10}$.

Final protocol developed in this trial required:

1. PAEROSOL being generated inside the test room over 30 minutes from $2.0 \mathrm{~L}$ of neutral $\mathrm{pH}$ solution of $2.5-5 \mathrm{~g} / \mathrm{L}$ table salt that has been electrochemically activated $(1 \mathrm{ml}$ of this solution was atomized to PAEROSOL per $1 \mathrm{ft}^{3}$ of the test room)

2. the test-room remained vacated for 4.0 hours total, including 30 minutes of PAEROSOL generation

3. the air handling system was shut down

4. the door was closed, but not sealed.

Effectiveness of the PAEROSOL application on reducing microbial viability on test samples was compared to their natural decline in viability on test coupons that were not exposed to PAEROSOL. The natural decline in viability of microorganisms was observed during 3 days in the test room and showed a generally insignificant natural decline of all microorganisms, except $P$. aeruginosa. In contrast, optimal PAEROSOL protocol killed 100 percent of the microorganisms on all materials except carpet during the 4-hour test period. It was demonstrated that there was no microbes' re-occurrence on decontaminated coupons during 48 hours after the contact with PAEROSOL.

The test room was located within a busy laboratory environment where occupants continued to work while PAEROSOL was tested inside the room. Fixtures, furniture, electrical, and electronic equipment stayed uncovered in the test room during PAEROSOL tests and remained fully operational through the trial and final observation. Neither visual deterioration nor damage resulting from their multiple exposures to PAEROSOL was observed. No visual deterioration was also witnessed on in-room inanimate surfaces, except some light rusting spots identified on two metal (not stainless steel) installations. Whether or not it was caused by regular PAEROSOL fumigation inside the room remains unclear. No discoloring or other damage was observed on wall posters, chair fabric, and on the paintedmetal frame of the chair that remained in the room through the trial. However, it was noted that the schematics drawn by markers on the floor and table surfaces, gradually faded during 4 months. It is not clear whether or not it was time-related discoloring or a PAEROSOL bleaching effect. No obvious 
damage (e.g., change in surface texture or color) or visible changes caused by PAEROSOL was exhibited in any of the test materials.

The room was disconnected from the HVAC system, and PAEROSOL penetration to the other MAMC facilities through a passive air outlet was controlled by covering outlets with cardboard. No additional safety precautions were taken, other than shutting off the air handing system and closing the door.

Because the passive air outlet was closed while the door was not sealed, PAEROSOL penetrated to the hallway underneath the door. $\mathrm{Cl}$ concentration, which constitutes total concentration of ROS in PAEROSOL, was monitored in the hallway and its concentration was below the detection limit $(<0.1$ ppm) when the test room door remained closed, though a light chlorine smell appeared in the hallway during tests. When questioned about the chlorine smell in the hallway, the DCI employees' response was always positive because the majority associated the light chlorine smell with cleanliness. Others either did not notice the smell, or it did not negatively affect them. PAEROSOL tests did not interfere with routine DCI functioning. No complaints from DCI employees were received.

PAEROSOL application required minimal equipment that was easy to operate; required little manpower, and left no waste other than micrograms of easily wiped-off salt on horizontal surfaces.

Though PAEROSOL efficacy was not evaluated by direct sampling of the inanimate surfaces within the hospital setting, using dosed coupons made from hospital surface materials and distributed within a typical hospital room provided a realistic condition for verification of the effectiveness of PAEROSOL.

We recognized certain limitations in the results received during PAEROSOL trial in MAMC. An experiment longer than 2 days is required to confirm a lack of microbes' re-occurrence on the materials decontaminated with PAEROSOL. The trial conducted at MAMC elucidated necessary modifications required for further optimization of PAEROSOL application. It would benefit PAEROSOL efficacy if $\mathrm{RH}$ in decontaminating room would not exceed 80 to 85 percent during PAEROSOL fumigation to prevent PAEROSOL particles colliding and to avoid moisture on room surfaces after decontamination completion. It could be achieved by VAG modification that would discontinue PAEROSOL production automatically when the RH in the room achieved desirable level. Computation modeling of the factors dictating PAEROSOL bactericidal efficacy is necessary to predict PAEROSOL efficacy in different environmental conditions, and differently sized facilities.

A distinction is made between preventive, current, and final disinfection. Preventive disinfection is carried out irrespective of the presence of infectious, sick people, to prevent diseases or their spread within groups. Current disinfection is performed in medical establishments to prevent spread of pathogens from sick people to those who come in contact with them. Final disinfection is done after the sick people have been discharged from the healthcare settings.

In general, in the healthcare settings preventive and current disinfections are hard to discriminate and therefore, a distinction is made between preventive (current) and final disinfections. For which of these two disinfections is PAEROSOL feasible? The results of the trial conducted in MAMC allowed us to conclude that PAEROSOL is practical for preventive and final disinfections. Minimal time and PAEROSOL volume will be required to reduce airborne pathogens (e.g., exhaled by sick person, etc., and re-aerosolized from inanimate surfaces). Such preventive PAEROSOL application will result in a 
reduced number of viable pathogens on inanimate surfaces and therefore, the final disinfection will require less time and lower volume of PAEROSOL for room treatment. The simplicity of PAEROSOL application and possibility to use it without precautions in the midst of busy hospital environment make PAEROSOL a prospective candidate to confront HAIs in the healthcare settings. 



\subsection{References}

Activated Environmental Solutions Inc. 2012. "Regulatory Approval.” Accessed April 19, 2012 at http://www.activatedenvironmentalsolutions.com/index.php/regulatory-approval.

Aggarwal R, N Goel, U Chaudhary, V Kumar, KP Ranjan. 2010. "Evaluation of microbiocidal activity of superoxidized water on hospital isolates." Indian Journal of Pathology and Microbiology, 53(4):757-759.

Anderl JN, MJ Franklin, and PS Stewart. 2000. "Role of antibiotic penetration limitation in Klebsiella pneumoniae biofilm resistance to ampicillin and ciprofloxacin." Antimicrobial Agents and Chemotherapy. 44(7):1818-1824.

Bakhir Institute of Electrochemical System and Technologies. 2012. Accessed April 23, 2012 at http://www.vbinstitute.ru/equipment/ (In Russian ).

Bakhir VM. 2012. "Electrochemical Systems and technologies." Accessed April 23, 2012 at http://www.bakhir.com.

Bakhir VM and YG Zadorozhny. 1997. "Electrochemical Cell.” US Patent 5,635,040.

Bakhir VM, BI Leonov, SA Panicheva, VI Prilutsky, and NY Shomovskaya. 2003. "Issues of chemical composition and operating properties of chlorine based inorganic liquid chemical germicides." Available at http://www.bakhir.com/publications/10-HClO-NaClO-Article.htm).

Barker J and MV Jones. 2005. "The potential spread of infection caused by aerosol contamination of surfaces after flushing a domestic toilet." Journal of Applied Microbiology, 99(2):339-347.

Berrington AW and S Pedler. 1998. "Investigation of gaseous ozone for MRSA decontamination of hospital side-rooms." The Journal of Hospital Infection, 40(1):61-65.

Bioquell. 2011. Hydrogen Peroxide Vapour (HPV) Biological Efficacy. Bioquell UK Ltd. Available at: http://www.bioquell.com/interface/downloads/069_11_HPV_Biological_Efficacy_UK.pdf.

Bioquell. 2012. "Hydrogen Peroxide Vapor Technology Decontamination Equipment and Services." Accessed April 18, 2012 at http://www.bioquellus.com.

Boyce JM. 2009. "New Approaches to Decontamination of Rooms After Patients Are Discharged." Infection Control and Hospital Epidemiology, 30(6).

Carling PC, J Briggs, D Hylander, and J Perkins. 2006. "An evaluation of patient area cleaning in 3 hospitals using a novel targeting methodology." American Journal of Infection Control, 34(8):513-519.

Carling, PC, MF Parry, and SM von Beheren. 2008a. "Identifying Opportunities to Enhance Environmental Cleaning in 23 Acute Care Hospitals." Infection Control and Hospital Epidemiology, 29(1):1-7. 
Carling PC, MM Parry, ME Rupp, JL Po, B Dick, S Von Beheren. 2008b. "Improving Cleaning of the Environment Surrounding Patients in 36 Acute Care Hospitals." Infection Control and Hospital Epidemiology, November, 29(11):1035-1041.

Carvalho, KS, MC Melo, GB Melo, PP Gontijo-Filho. 2007. "Hospital surface contamination in wards occupied by patients infected with MRSA or MSSA in a Brazilian university hospital." Revista de Ciências Farmacêuticas Básica e Aplicada, (Journal of Basic and Applied Pharmaceutical Sciences) 28(2):159-163.

Centers for Disease Control. 1972. Disinfectant fogging, an ineffective measure. NNIS report 1971 (third quarter), DHEW Publication No. CDC 72-8149, United States Government Printing Office, Washington, D.C.

Centers for Disease Control. 2002. "CDC's Campaign to Prevent Antimicrobial Resistance in HealthCare Settings.” Morbidity and Mortality Weekly Report (MMWR) 51(15)343.

Clark J, SP Barrett, M Rogers, and R Stapleton. 2006. "Efficacy of super-oxidized water fogging in environmental decontamination." Journal of Hospital Infection 64, 386-390.

Cloete TE, MS Thantsha, MR Maluleke, and R Kirkpatrick, R. 2009. "The antimicrobial mechanism of electrochemically activated water against Pseudomonas aeruginosa and Escherichia coli as determined by SDS-PAGE analysis." Journal of Applied Microbiology, 107(2):379-384.

Davies A, T Pottage, A Bennett, J Walker. 2011. "Gaseous and air decontamination technologies for Clostridium difficile in the healthcare environment." Journal of Hospital Infection, 77(3):199-203.

de Boer HE, CM van Elzelingen-Dekker, CM van Rheenen-Verberg, and L Spanjaard. 2006. "Use of gaseous ozone for eradication of methicillin-resistant Staphylococcus aureus from the home environment of a colonized hospital employee." Infection Control and Hospital Epidemiology, 27(10):1120-1122.

Department of Health/NHS Purchasing and Supply Agency. 2009. The Results, Using technology to help fight infection, HCAI Technology Innovation Programme, Showcase Hospitals report number 3, The Bioquell Hydrogen Peroxide Vapour (HPV) Disinfection System. Available at http://hcai.dh.gov.uk/files/2011/03/090817_HCAI_Technology_Innovation_Programme_Showcase_Hosp itals_Report_3_The_Bioquell_Hydrogen_Peroxide_Vapour_HPV_Disinfection_System.pdf

Deza MA, M Araujo, and MJ Garrido. 2003.’Inactivation of Escherichia coli O157:H7, Salmonella enteriditis and Listeria monocytogenes on the surface of tomatoes by neutral electrolyzed water." Letters in Applied Microbiology, 37(6):482-487.

Donlan RM and JW Costerton. 2002. "Biofilms: Survival Mechanisms of Clinically Relevant Microorganisms.” Clinical Microbiology Reviews, 15(2):167-193.

Drees M, DR Snydman, CH Schmid, L Barefoot, and K Hansjosten. 2008. "Prior environmental contamination increases the risk of acquisition of vancomycin-resistant enterococci." Clinical Infectious Diseases, 46(4):678-685.

Envirolyte. 2012. “Technology” http://www.envirolyteindia.com) 
EPA. 2005. Compilation of Available Data on Building Decontamination Alternatives. EPA/600/R05/036, U.S. Environmental Protection Agency, National Homeland Security Research Center, Washington, D.C.

European Centre for Disease Prevention and Control. 2010. Risk assessment guidelines for diseases transmitted on aircraft. 2nd ed. European Centre for Disease Prevention and Control Stockholm. Available at http://ecdc.europa.eu/en/publications/Publications/1012_GUI_RAGIDA_2.pdf.

Favero MS. 2004. "Naturally occurring microrganisms and their resistance to physical and chemical agents." In: Disinfection, sterilization and antisepsis: Principles, practices, challenges, and new research. WA Rutala, ed., 1-14, Association for Professionals in Infection Control and Epidemiology, Washington D.C.

Fisher J, RA Caputo. 2004. "Comparing and Contrasting Barrier Isolator Decontamination Systems." Pharmaceutical Technology, 28(11):68-82.

Flanders SA, HR Collard, and S Saint. 2006. "Nosocomial pneumonia: state of the science." American Journal of Infection Control, 34(2):84-93.

The Free Library by Farlex. 2012. "Department of Defense Begins Testing Electro-Chemical Technologies ECASOL Fog." Accessed April 19, 2012 at http://www.thefreelibrary.com/Department+of+Defense+Begins+Testing+ElectroChemical+Technologies...-a094808229.

Grosser M. 2001. "Statement of Mr. Mike Grosser, United States Marine Corps Technical Director, Program Manager, Nuclear, Biological and Chemical Defense Systems Marine Corps Systems Command Before the Committee on Environment and Public Work, United States Senate, 4 December 2001, Concerning Technologies Available for Remediating Buildings Contaminated by Biological Contaminants. Available at http://epw.senate.gov/107th/Grosser_1204.htm

Hayden MK, MJM Bonten, DW Blom, EA Lyle, DAMC van de Vijver, and RA Weinstein. 2006. "Reduction in Acquisition of Vancomycin-resistant Enterococcus after Enforcement of Routine Environmental Cleaning Measures." Clinical Infectious Diseases, 42 (11):1552-1560.

Hidron AI, JR Edwards, J Patel, TC Horan, SM Sievert, DA Pollock, SK Fridkin, the National Healthcare Safety Network Team, and Participating National Healthcare Safety Network Facilities. 2008. "NHSN Update: Antimicrobial-Resistant Pathogens Associated With Healthcare-Associated Infections: Annual Summary of Data Reported to the National Healthcare Safety Network at the Centers for Disease Control and Prevention, 2006-2007." Infection Control and Hospital Epidemiology, 29(11): 996-1011.

Huang SS, R Datta, and R Platt 2006. "Risk of acquiring antibiotic- resistant bacteria from prior room occupants." Archives of Internal Medicine, 166(18):1945-51.

Jain A and K Singh. 2007. "Recent Advances in the Management of Nosocomial Infections." JK Science, 9(1):3-8.

Jeng DK and AG Woodworth. 1990. "Chlorine Dioxide Gas Sterilization under Square-Wave Conditions.” Applied and Environmental Microbiology, 56(2):514-519. 
Jeong J, JY Kim, and J Yoon. 2006. "The role of reactive oxygen species in the electrochemical inactivation of microorganisms.” Environmental Science and Technology, 40(19): 6117-6122.

Kak, V. 2007. "Infections in Confined Spaces: Cruise Ships, Military Barracks, and College Dormitories." Infectious Disease Clinics of North America, 21(3):773-784.

Kramer A, I Schwebke, and G Kampf. 2006. "How long do nosocomial pathogens persist on inanimate surfaces? A systematic review.” BMC Infectious Diseases, 6:130, doi:10.1186/1471-2334-6-130.

Len SV, YC Hung, D Chung, JL Anderson, MC Erickson, and K Morita. 2002. "Effects of storage conditions and $\mathrm{pH}$ on chlorine loss in electrolyzed oxidizing (EO) water." Journal of Agricultural and Food Chemistry, 50(1):209-212.

Luftman HS, MA Regits, P Lorcheim, MA Czarneski, T Boyle, H Aceto, B Dallap, D Munro, and K Faylor. 2004. "Chlorine Dioxide Gas Decontamination of Large Animal Hospital Intensive and Neonatal Care Units.” doi=10.1.1.163.7122.

Maillard J-Y. 2005. "Antimicrobial biocides in the healthcare environment: efficacy, usage, policies, and perceived problems.” Therapeutics and Clinical Risk Management, 1(4):307-320.

Marais JT. 2000. "Cleaning efficacy of a new root canal irrigation solution: a preliminary evaluation." International Endodontic Journal, 33(4):320-325.

Marais JT and VS Brozel. 1999. "Electro-chemically activated water in dental unit water lines." British Dental Journal, 187(3):154-158.

Marais JT and WP Williams. 2001. "Antimicrobial effectiveness of electro-chemically activated water as an endodontic irrigation solution.” International Endodontic Journal, 34(3): 237-243.

McAnoy AM. 2006. Vaporous decontamination methods: potential uses and research priorities for chemical and biological contamination control. DSTO-GD-0465, Australian Government, Department of Defence, Accessed April 18, 2012 at http://www.dsto.defence.gov.au/publications/scientific_record.php?record=3415.

McDonald LC, M Owings, and DB Jernigan. 2006. "Clostridium difficile infection in patients discharged from US Short-stay Hospitals, 1996-2003.” Emerging Infectious Diseases, 12(3):409-415.

Memarzadeh F. 2012. "The Environment of Care and Health Care-Associated Infections: An Engineering Perspective.” American Society for Healthcare Engineering (ASHE) Monograph. Accessed March 30, 2012 at http://www.ashe.org/resources/management monographs/mg2011memarzadeh.html.

MIOX. 2011. "What Is Mixed Oxidant Solution?” MIOX, Albuquerque, New Mexico. Available at http://www.miox.com/Files/What\%20is\%20MOS_twopager_112311.pdf.

Moat J, J Cargill, J Shone, and M Upton. 2009. "Application of a novel decontamination process using gaseous ozone.” Canadian Journal of Microbiology, 55(8):928-933. 
Neely AN. 2012. "Persistence of microorganisms on common hospital surfaces, Strategies to control their dissemination." Infection Control Resource, 4(4). Accessed April 26, 2012 at http://www.infectioncontrolresource.org/Past_Issues/IC16.pdf.

Nogler M, C Lass-Flörl, M Ogon, E Mayr, C Bach, and C Wimmer. 2001. "Environmental and Body Contamination Through Aerosols Produced by High-Speed Cutters in Lumbar Spine Surgery." Spine, 26(19):2156-2159.

O’Donnell MJ, M Boyle, J Swan, RJ Russell, and DC Coleman. 2009 . "A centralised, automated dental hospital water quality and biofilm management system using neutral Ecasol ${ }^{\mathrm{TM}}$ maintains dental unit waterline output at better than potable quality: A 2-year longitudinal study." Journal of Dentistry, 37(10):748-762.

Otter JA and GL French. 2009. "Survival of Nosocomial Bacteria and Spores on Surfaces and Inactivation by Hydrogen Peroxide Vapor.” Journal of Clinical Microbiology, 47(1):205-207.

Park GW, DM Boston, JA Kase, MN Sampson, and MD Sobsey. 2007. "Evaluation of Liquid- and FogBased Application of Sterilox Hypochlorous Acid Solution for Surface Inactivation of Human Norovirus." Applied and Environmental Microbiology, 73(14):4463-4468.

Pettit F and EJL Lowbury. 1968. "Survival of wound pathogens under different environmental conditions." Journal of Hygiene, Cambridge, 66(3): 393-406.

Prilutsky VI and VM Bakhir. 1997. "Electrochemically activated water: anomalous properties, mechanism of biological action." VNIII of Medical Engineering, p.232. Reference from http://www.bakhir.com/other/leonov_article/, accessed April 23, 2012.

Puricore. 2012. "PuriCore Launches Vashe Wound Therapy for Instillation Applications" Accessed April 19, 2012 at http://www.puricore.com/PDFs/PuriCoreVasheInstillation.pdf

Qian J, D Hospodsky, N Yamamoto, WW Nazaroff, and J Peccia. 2012. "Size-resolved emission rates of airborne bacteria and fungi in an occupied classroom." Indoor Air. doi: 10.1111/j.1600-

0668.2012.00769.x

Rautemaa R, A Nordberg, K Wuolijoki-Saaristo, JH Meurman. 2006. "Bacterial aerosols in dental practice - a potential hospital infection problem?” Journal of Hospital Infection, 64(1):76-81.

Rogers JV, GR Ducatte, YW Choi, and PC Early, P.C. 2006. A preliminary assessment of Bacillus anthracis spore inactivation using an electrochemically activated solution $\mathrm{ECASOL}^{\mathrm{TM}}$ ). Letters in Applied Microbiology, 43:482-488.

Rosenblatt DH, AA Rosenblatt, and JE Knapp. 1985. "Use of chlorine dioxide gas as a chemosterilizing agent." US Patent 4,504,442.

Rosenblatt DH, AA Rosenblatt, and JE Knapp. 1987. "Use of chlorine dioxide gas as a chemosterilizing agent." US Patent 4,681,739. 
Sexton T, P Clarke, E O’Neill, T Dillane, and H Humphreys. 2006. "Environmental reservoirs of methicillin-resistant Staphylococcus aureus in isolation rooms: correlation with patient isolates and implications for hospital hygiene." Journal of Hospital Infection, 62(2):187-194, DOI:

10.1016/j.jhin.2005.07.017.

Sharma M and JB Hudson. 2008. "Ozone gas is an effective and practical antibacterial agent." American Journal of Infection Control, 36(8):559-563.

Shiomori T, H Miyamotoy, K Makishima, M Yoshida, T Fujiyoshi, T Udaka, T Inaba, and N Hiraki. 2002. "Evaluation of bedmaking-related airborne and surface methicillin-resistant Staphylococcus aureus contamination." Journal of Hospital Infection, 50(1):30-35.

Sigua G, Y-H Lee, J Lee, K Lee, J Hipp , and MA Pascall. 2011. "Comparative efficacies of various chemical sanitizers for ware washing operations in restaurants." Food Control 22:130-19.

Skaliy P and RG Eagon. 1972. "Effect of Physiological Age and State on Survival of Desiccated Pseudomonas aeruginosa." Applied and Environmental Microbiology, 24(5):763-767.

Solovyeva, AM and PM Dummer. 2000. "Cleaning effectiveness of root canal irrigation with electrochemically activated anolyte and catholyte solutions: a pilot study." International Endodontic Journal, 33(6):494-504.

STERIS. 2012. "Vaporized Hydrogen Peroxide (VHP®) Biodecontamination Systems." Accessed on April 23, 2012 at http://www.sterislifesciences.com/Products/Equipment/VHP.aspx.

Sventitsky E, E Rainina, V Gluschenko, Y Tolparov, T Egorova, N Kontorina, V Iskritskyi. Microaerosol-based decontamination method PCT/US09/48765; PCT/RU2008/000782).

Trustwater. 2011. 2011 NCIMS Conference PMO Scientific Data. Available at http://www.adph.org/foodsafety/assets/NCMISPMOScientificData.pdf.

Urbina, I. 2007. "Schools in Several States Report Staph Infections, and Death Raise the Alarm." The New York Times. Accessed March 30, 2012 at http://www.nytimes.com/2007/10/19/us/19staph.html?_r=1\&scp=292\&sq=\&st=nyt.

Valentine NB, MG Butcher, Y-F Su, KH Jarman, M. Matzke, B-J Webb-Robertson, EA Panisko, BAB Seiders and KL Wahl. 2008. "Evaluation of sampling tools for environmental sampling of bacterial endospores from porous and nonporous surfaces." Journal of Applied Microbiology 105(4):1107-1113.

Venkitanarayanan KS, GO Ezeike, Y Hung, and MP Doyle. 1999. "Efficacy of electrolyzed oxidizing water for inactivating Escherichia coli O157:H7, Salmonella enteritidis, and Listeria monocytogenes." Applied and Environmental Microbiology, 65(9):4276-4279.

Wainwright CE, MW France, P O’Rourke, S Anuj, TJ Kidd, MD Nissen, TP Sloots, C Coulter, Z Ristovski, M Hargreaves, BR Rose, C Harbour, SC Bell, KP Fennelly. 2009. "Cough-generated aerosols of Pseudomonas aeruginosa and other Gram-negative bacteria from patients with cystic fibrosis." Thorax, 64: 926-931; 
Weinberg HS, A Sykes, and S Rodriguez-Mozaz. 2008. Characterization of the Chemical Constituents of Mixed Oxidant Disinfection. Final Project Report, presented to MIOX Corporation by the University of North Carolina, Department of Environmental Sciences and Engineering, Chapel Hill, NC, 23 July 2008; Note: This is not publicly available see http://www.unc.edu/ weinberg/research/2008characterization.shtml

Wilcox MH, and WN Fawley. 2000. "Hospital disinfectants and spore formation by Clostridium difficile." Lancet, 356(9238):1324.

Wilder-Smith A and HN Leong. 2004. "A case of in-flight transmission of severe acute respiratory syndrome (SARS): SARS serology positive.” Journal of Travel Medicine, 11(2):130.

Zoutman D, M Shannon, and A Mandel. 2011. Effectiveness of a novel ozone-based system for the rapid high-level disinfection of health care spaces and surfaces. American Journal of Infection Control, 39(10):873-879. 


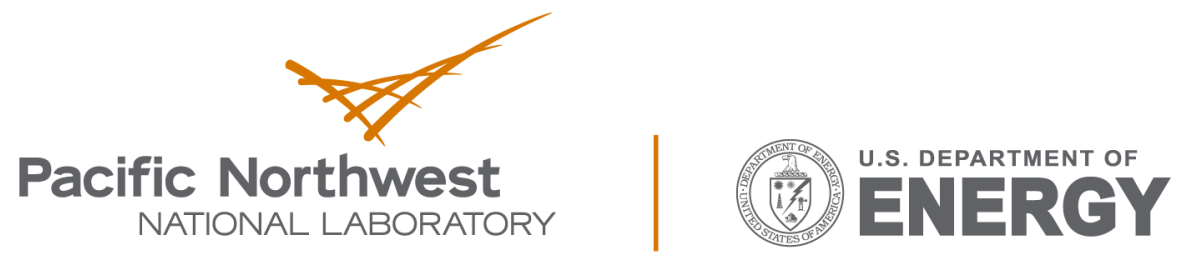

Proudly Operated by Battelle Since 1965

902 Battelle Boulevard

P.O. Box 999

Richland, WA 99352

1-888-375-PNNL (7665)

www.pnnl.gov 